\title{
Search for the production of four top quarks in the single-lepton and opposite-sign dilepton final states in proton-proton collisions at $\sqrt{s}=13 \mathrm{TeV}$
}

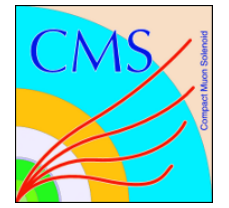

\section{The CMS collaboration}

E-mail: cms-publication-committee-chair@cern.ch

ABStract: A search for the standard model production of four top quarks ( $\mathrm{pp} \rightarrow \mathrm{t} \overline{\mathrm{t}} \mathrm{t} \overline{\mathrm{t}}$ ) is reported using single-lepton plus jets and opposite-sign dilepton plus jets signatures. Proton-proton collisions are recorded with the CMS detector at the LHC at a center-ofmass energy of $13 \mathrm{TeV}$ in a sample corresponding to an integrated luminosity of $35.8 \mathrm{fb}^{-1}$. A multivariate analysis exploiting global event and jet properties is used to discriminate $t \bar{t} t \bar{t}$ from $t \bar{t}$ production. No significant deviation is observed from the predicted background. An upper limit is set on the cross section for t tett production in the standard model of $48 \mathrm{fb}$ at $95 \%$ confidence level. When combined with a previous measurement by the CMS experiment from an analysis of other final states, the observed signal significance is 1.4 standard deviations, and the combined cross section measurement is $13_{-9}^{+11} \mathrm{fb}$. The result is also interpreted in the framework of effective field theory.

KEYwords: Hadron-Hadron scattering (experiments), Top physics

ARXIV EPRINT: 1906.02805 


\section{Contents}

1 Introduction $\quad 1$

2 The CMS detector 2

3 Simulated samples 3

4 Data analysis 4

4.1 Event selection 4

$\begin{array}{lll}4.2 & \text { Multivariate discriminants } & 6\end{array}$

$\begin{array}{llr}5 & \text { Systematic uncertainties } & 8\end{array}$

6 Results $\quad 14$

6.1 Combination with the same-sign dilepton and multileptons channels 22

6.2 Effective field theory interpretation 23

$\begin{array}{lll}7 & \text { Summary } & 25\end{array}$

$\begin{array}{ll}\text { The CMS collaboration } & 33\end{array}$

\section{Introduction}

Many models of physics beyond the standard model (BSM) predict enhanced or modified couplings of top quarks to other particles. This is particularly relevant for processes that have small production cross sections and, therefore, are yet to be observed, such as the production of four top quarks, $t \bar{t} t \bar{t}$. There is considerable interest in the measurement of the $t \bar{t} t \bar{t}$ cross section because of its sensitivity to BSM physics, including supersymmetry [1, 2], composite models [3], top quark compositeness [4], two-Higgs-doublet models [5-7], and models with extra spatial dimensions $[8,9]$. Within the effective field theory (EFT) framework, the contribution of any BSM process to $t \overline{\mathrm{t}} \mathrm{t} \overline{\mathrm{t}}$ production can be parameterized in terms of nonrenormalizable effective couplings of the standard model (SM) fields, if the characteristic energy scale, $\Lambda$, of the BSM physics is much larger than the typical energy scale of $t \bar{t} t \bar{t}$ production at the LHC. A generic interpretation of the $t \bar{t} t \bar{t}$ production can be done using the EFT predictions [10].

The production of four top quarks from proton-proton $(\mathrm{pp})$ interactions $\mathrm{pp} \rightarrow \mathrm{t} \overline{\mathrm{t}} \mathrm{t} \overline{\mathrm{t}}$ has not yet been observed. The SM predicts a cross section, at next-to-leading order (NLO), with electroweak corrections (EWK), of $\sigma_{\mathrm{t} \overline{\mathrm{t}} \mathrm{t} \overline{\mathrm{t}}}^{\mathrm{SM}}$ of $12.0 \mathrm{fb}$ at the center-of-mass energy of $13 \mathrm{TeV}$ [11]. To facilitate comparison with published ATLAS and CMS analyses using comparable data sets, the NLO quantum chromodynamics (QCD) calculation with a value 

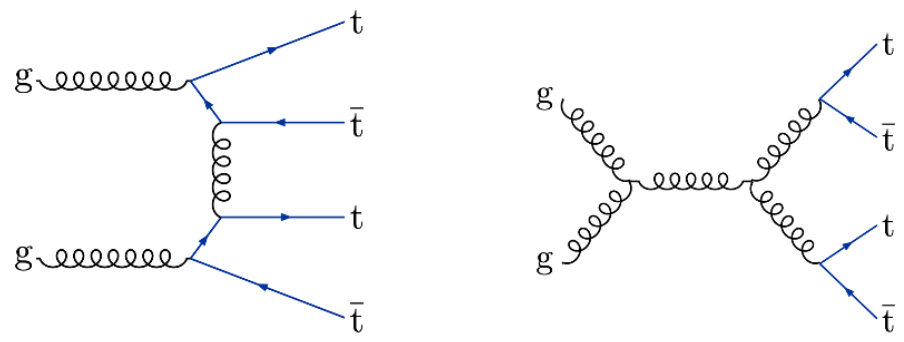

Figure 1. Representative Feynman diagrams for $\mathrm{pp} \rightarrow \mathrm{t} \overline{\mathrm{t}} \overline{\mathrm{t}}$ production at lowest order in the SM.

of $\sigma_{\mathrm{t} \overline{\mathrm{t}} \mathrm{t}}^{\mathrm{SM}}=9.2 \mathrm{fb}$ is used $[12,13]$. Consequently, the experiments at the CERN LHC may be just approaching sensitivity to the process, provided that it can be separated from the overwhelming background from SM t $\bar{t}$ events. The lowest-order Feynman diagrams illustrating typical contributions to SM four top quark production in pp collisions are shown in figure 1.

Searches for $t \bar{t} t \bar{t}$ production have been performed at $8 \mathrm{TeV}$ by ATLAS [14-16] and CMS [17], and also at $13 \mathrm{TeV}$ (ATLAS $\left(36.1 \mathrm{fb}^{-1}[18,19]\right)$ and CMS $\left.\left(2.6 \mathrm{fb}^{-1}[20]\right)\right)$. The CMS Collaboration measured the $t \bar{t} t \bar{t}$ production cross section in a search exploiting samesign dilepton and multilepton signatures [21, 22] using $13 \mathrm{TeV}$ data $\left(35.9 \mathrm{fb}^{-1}\right)$ collected in 2016. The ATLAS Collaboration investigated anomalous t $\bar{t} t \bar{t}$ production in events with Lorentz-boosted top quarks identified with top quark tagging techniques [23] using $13 \mathrm{TeV}$ data $\left(36.1 \mathrm{fb}^{-1}\right)$ collected in $2015-2016$.

This paper presents a new search in the single-lepton (SL) $(\mu$, e)+jets and oppositesign dilepton (DL) $\left(\mu^{+} \mu^{-}, \mu^{ \pm} \mathrm{e}^{\mp}\right.$, or $\left.\mathrm{e}^{+} \mathrm{e}^{-}\right)+$jets $\mathrm{t} \overline{\mathrm{t}} \mathrm{t} \overline{\mathrm{t}}$ decay channels using pp collisions at $13 \mathrm{TeV}$ collected by the CMS experiment in 2016 and corresponding to an integrated luminosity of $35.8 \mathrm{fb}^{-1}$. For this analysis, only final states containing one or two leptons are considered, which constitute about $40 \%$ of all $t \bar{t} t \bar{t}$ decays. Compared to the previous analysis [20], we have implemented a number of important changes which combine to give a much improved analysis sensitivity. The training process and selection of the input variables for the event-discriminating MVA's (section 4.2) in both the SL and OS dilepton channels has been re-optimized. A new categorization of the signal sensitive regions at large jet and b-tag multiplicities has been introduced, and a revised binning scheme is used to decrease the statistical uncertainties, and improve the signal sensitivity. The categorisation provides additional discrimination against the rare $t \overline{\mathrm{t}}+$ boson $(\mathrm{H}, \mathrm{Z}, \mathrm{W}, \mathrm{WW} / \mathrm{WZ} / \mathrm{ZZ})$ backgrounds. Lastly, a much larger simulated $t \bar{t}$ data set is used to populate the discriminant bins with high jet multiplicity and high b-tag multiplicity.

\section{The CMS detector}

The central feature of the CMS apparatus is a superconducting solenoid of $6 \mathrm{~m}$ internal diameter, providing a magnetic field of $3.8 \mathrm{~T}$. Within the solenoid volume are a silicon pixel and strip tracker, a lead tungstate crystal electromagnetic calorimeter, and a brass and scintillator hadron calorimeter, each composed of a barrel and two endcap sections. 
Forward calorimeters extend the pseudorapidity coverage $(\eta)$ provided by the barrel and endcap detectors. Muons are measured in gas-ionization detectors embedded in the steel flux-return yoke outside the solenoid, in the range $|\eta|<2.4$. A more detailed description of the CMS detector, together with a definition of the coordinate system used and the relevant kinematic variables, can be found in ref. [24].

\section{Simulated samples}

The acceptance for the SM pp $\rightarrow \mathrm{t} \overline{\mathrm{t}} \mathrm{t} \overline{\mathrm{t}}$ process is estimated using samples simulated at NLO precision by the MADGRAPH5_aMC@NLO 2.2.2 generator [13, 25]. Only diagrams arising from quantum chromodynamics interactions were taken into account in the simulation. The cross section used to normalize the simulation is the NLO calculation of $9.2_{-2.4}^{+2.9}$ $\mathrm{fb}$ [13], where the quoted uncertainty incorporates the variation of factorization and renormalization scales used in the calculation of the matrix elements (ME), and the dependence on the choice of parton distribution functions (PDFs). The signal model includes MADSpIN [26] and uses the default dynamic scale choice in MADGRAPH5_aMC@NLO, defined as $\mu_{\mathrm{R}, \mathrm{F}}=\frac{1}{2} \Sigma_{\mathrm{t}} m_{\mathrm{T}}$. This is the sum of $m_{\mathrm{T}}$ over each outgoing parton (the four top quarks), divided by two, where $m_{\mathrm{T}}=\sqrt{m^{2}+p_{\mathrm{T}}^{2}}$, in which $m$ is the mass of the parton, and $p_{\mathrm{T}}$ is the transverse momentum.

The most important background process is top quark pair production with additional jets ( $\mathrm{t} \overline{\mathrm{t}}+\mathrm{jets})$, that comprises over $90-95 \%$ of the background. Next in importance are single top (ST) quark processes including $t$-channel and $\mathrm{tW}$ production. These are followed by $\mathrm{Z}+$ jets and $\mathrm{W}+$ jets electroweak processes $(\mathrm{EW})$, where only the leptonic decay modes of the bosons are considered. Next are rare processes involving the production of a top quarkantiquark pair and a Z, W, or Higgs bosons, namely, $t \overline{\mathrm{t}}+\mathrm{Z}, \mathrm{W}, \mathrm{H}$. Finally, $\mathrm{t} \overline{\mathrm{t}}$ production in association with dibosons, $t \bar{t} W W, t \bar{t} W Z, t \bar{t} Z Z, t \bar{t} W H, t \bar{t} Z W, t \bar{t} H H$, and triple top quark production ( $\mathrm{t} \overline{\mathrm{t}} \mathrm{t}+\mathrm{jets}$ and $\mathrm{t} \overline{\mathrm{t}} \mathrm{tW})$ are considered, processes we collectively denote as $t \bar{t} X Y$. Based on their signature resemblance and comparability of production rates to the $t \bar{t} t \bar{t}$ signal, $t \bar{t}+Z$ and $t \bar{t}+H$ are grouped together while $t \bar{t}+W$ and $t \bar{t} X Y$ are grouped together in the simulation.

Several Monte Carlo (MC) event generators are used to simulate these processes. The $\mathrm{t} \overline{\mathrm{t}}+$ jets process is simulated using the POWHEG-BOX v2 generator [27-31] at NLO accuracy for the $t \overline{\mathrm{t}} \mathrm{ME}$, but the $\mathrm{t} \overline{\mathrm{t}}$ cross section is normalized to its predicted value at next-to-next-to-leading order (NNLO), which includes soft-gluon corrections, at next-tonext-to-leading-logarithm accuracy, obtained with Top ++2.0 [32-38]. The POWHEG-BOX simulations are interfaced with PYTHIA 8.212 using the CUETP8M2T4 tune [39-41]. Recent calculations [38] suggest that next-to-next-to-leading-order effects have an important consequence on the shape of the top quark $p_{\mathrm{T}}$ spectrum that NLO ME generators are

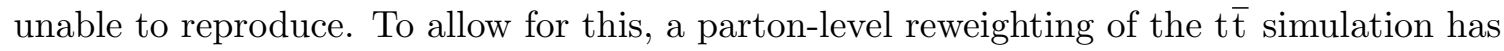
been applied to match the predictions to the data [42, 43]. The correction is applied as a function of the transverse momenta of the parton-level top quark and antiquark after initial- and final-state radiation. Specifically for this result, additional dedicated samples were created that populate the tails in high multiplicity with a factor of 10 more events. 
Single top quark tW processes are simulated with the POWHEG-BOX v1 generator [44], while $t$-channel processes are simulated with POWHEG-BOX v2. Both are interfaced with PYTHIA 8.212 using the CUETP8M2T4 tune, with the cross sections normalized to the NLO calculations $[45,46]$. The analysis has been shown [20] to be insensitive to other ST quark production processes, such as $s$-channel production.

Events with massive gauge bosons and no top quarks $(\mathrm{Z}+$ jets, $\mathrm{W}+$ jets $)$ are simulated using MADGRAPH5_aMC@NLO [13] at leading-order (LO) accuracy, with up to four additional partons in the ME calculations, and using the MLM matching scheme [47]. The tune CUETP8M1 is used for the parton shower (PS) and underlying event (UE) modeling. These samples are normalized to their NNLO cross sections [48].

The production of a t $\bar{t}$ pair in association with a $\mathrm{W}, \mathrm{Z}$ and up to one extra parton is simulated using the MADGRAPH5_aMC@NLO generator at LO accuracy and matched with the PS predictions using the MLM matching scheme. Top quark pair production in association with a Higgs boson, $\mathrm{t} \overline{\mathrm{t}} \mathrm{H}$, is modeled using POWHEG-BOX v2, interfaced with PYTHIA 8.212 with the CUETP8M2T4 tune. In this sample, only the dominant $\mathrm{H} \rightarrow \mathrm{b} \overline{\mathrm{b}}$ decays are taken into account. These three samples are normalized to the NLO cross sections [49]. Top quark pair production in association with one or two massive bosons is simulated using the LO ME in the MADGRAPH5_aMC@NLO generator, and the CUETP8M2T4 tune of PYTHIA 8.212 to provide the PS. The cross sections are scaled to their LO values [49].

For the samples with NLO MEs, the NNPDF3.0NLO [50] PDFs are used, while for LO MEs, the corresponding NNPDF3.0LO PDFs are used. The parton shower, hadronization, and underlying event models implemented in PYTHIA 8.212 [51] are used to simulate higherorder processes and nonperturbative aspects of pp collisions. The NLO simulations use strong coupling constant values of $\alpha_{S}\left(M_{\mathrm{Z}}\right)=0.137$ and $\alpha_{S}\left(M_{\mathrm{Z}}\right)=0.113$ for the ME and PS modeling, and the LO simulations use $\alpha_{S}\left(M_{\mathrm{Z}}\right)=0.130$ for the ME. In all simulations involving the top quark, a mass $m_{\mathrm{t}}$ of $172.5 \mathrm{GeV}$ is used.

The PYTHIA CUETP8M2T4 tune [39-41] currently provides the best description of the $\mathrm{t} \overline{\mathrm{t}}$ data $[52,53]$. The POWHEG-BOx calculation describes the high-multiplicity tail when this tune is used. The uncertainties cover the differences due to alternative choices of the PS and hadronization models [54].

All of the simulated samples include an estimate of the additional pp interactions per bunch crossing (pileup), modeled with the PYTHIA 8.212 program. Corrections are applied to make the simulation of the number of additional interactions representative of that observed in the data. The simulated events are propagated through a simulation of the CMS detector based on GEANT4 (v.9.4) [55] and reconstructed using the same algorithms as for the collider data.

\section{Data analysis}

\subsection{Event selection}

The final states considered in this analysis are the single-lepton channel with exactly one muon or electron, $(\mu, \mathrm{e})+$ jets, and the opposite-sign dilepton channel, $\left(\mu^{+} \mu^{-}, \mu^{ \pm} \mathrm{e}^{\mp}\right.$, 
$\left.\mathrm{e}^{+} \mathrm{e}^{-}\right)+$jets. In all cases, the leptons are expected to originate from the $\mathrm{W}$ bosons arising from top quark decays and thus tend to be isolated, unlike the leptons produced in the decay of unstable hadrons within jets.

Single-lepton events were recorded using a trigger [56] that required at least one isolated muon with $p_{\mathrm{T}}>24 \mathrm{GeV}$ and $|\eta|<2.4$, or one isolated electron with $p_{\mathrm{T}}>32 \mathrm{GeV}$ and $|\eta|<2.1$. Dilepton events were recorded using either single-lepton or dilepton triggers. In the case of dilepton triggers, the $p_{\mathrm{T}}$ thresholds for the leading and subleading leptons for the dimuon triggers are 17 and $8 \mathrm{GeV}$, respectively, 23 and $12 \mathrm{GeV}$ for dielectron triggers, and 23 and $8 \mathrm{GeV}$ for muon-electron triggers, regardless of lepton flavor. Dilepton triggers require $|\eta|<2.4$ for muons and $|\eta|<2.5$ for electrons. The single-lepton triggers were also used in the dilepton channel to increase the efficiency, while retaining the orthogonality of the selections addressing the two final states.

Offline event reconstruction uses the CMS particle-flow (PF) algorithm [57] for particle reconstruction and identification. Single-lepton events are required to have exactly one isolated muon with $p_{\mathrm{T}}>26 \mathrm{GeV}$ or one isolated electron with $p_{\mathrm{T}}>35 \mathrm{GeV}$, either within $|\eta|<2.1$. In the dilepton channel, events are required to contain exactly two isolated leptons of opposite sign with $p_{\mathrm{T}}>25 \mathrm{GeV}$ for the leading and $p_{\mathrm{T}}>20 \mathrm{GeV}$ for the subleading lepton, within $|\eta|<2.4$. Muons must satisfy the criteria described in ref. [58] and have a relative isolation, $I_{\text {rel }}<0.15$. Electron candidates must satisfy stringent identification criteria, including $I_{\text {rel }}$, which are described in ref. [59]. The $I_{\text {rel }}$ is defined as the scalar $p_{\mathrm{T}}$ sum of the additional particles consistent with the same vertex as the lepton, within a cone of angular radius $\Delta R=\sqrt{(\Delta \eta)^{2}+(\Delta \phi)^{2}}=0.4$ around the lepton, divided by the $p_{\mathrm{T}}$ of the lepton, where $\Delta \eta$ and $\Delta \phi$ (in radians) are the differences in pseudorapidity and azimuthal angle, respectively, between the directions of the lepton and the additional particle. The sum is corrected for the neutral particle contribution from pileup on an event-by-event basis [58, 59]. To suppress background events from decays of low-mass resonances and $\mathrm{Z}$ bosons, the lepton pairs are required to have an invariant mass greater than $20 \mathrm{GeV}$ and be outside of a $30 \mathrm{GeV}$ window centered on the $\mathrm{Z}$ boson mass in both the $\mu^{+} \mu^{-}$and $\mathrm{e}^{+} \mathrm{e}^{-}$ channels. Events containing additional muons with looser relative isolation, $I_{\text {rel }}<0.25$, or isolated electrons are vetoed.

Each event is required to contain at least one reconstructed vertex. The reconstructed vertex with the largest value of the quadratic sum of the $p_{\mathrm{T}}$ of its associated tracks is considered the primary pp interaction vertex. Jets are reconstructed from the PF candidates using the infrared- and collinear-safe anti- $k_{\mathrm{T}}$ algorithm $[60,61]$ with a distance parameter of 0.4. Pileup interactions can contribute tracks and calorimetric energy depositions to the jet momentum. To mitigate this effect, charged particles identified as originating from pileup vertices are discarded and the jet is corrected for the remaining contributions [62, 63]. Jet energy corrections are derived from simulations to bring the measured response of jets to that of particle level jets on average. In situ measurements of the momentum balance in dijet, photon+jet, $\mathrm{Z}+$ jet, and multijet events are used to account for any residual differences in jet energy scale between real and simulated data [64]. The jet energy resolution is typically $15 \%$ at $10 \mathrm{GeV}, 8 \%$ at $100 \mathrm{GeV}$, and $4 \%$ at $1 \mathrm{TeV}$. The missing transverse momentum vector $\vec{p}_{\mathrm{T}}^{\text {miss }}$ is computed as the negative vector sum of $p_{\mathrm{T}}$ of all the PF candidates 
in an event, and its magnitude is denoted as $p_{\mathrm{T}}^{\text {miss }}[65]$. The quantity $\vec{p}_{\mathrm{T}}^{\text {miss }}$ is modified to account for corrections to the energy of the reconstructed jets in the event.

A minimum of seven jets for the single-muon and eight jets for the single-electron channel are required, each of which must have $p_{\mathrm{T}}>30 \mathrm{GeV}$ and $|\eta|<2.5$. The difference in the jet multiplicity is motivated by the need to reduce the residual contamination from multijet QCD background in the electron channel due to a higher lepton misidentification rate. In the selected events, at least two jets must be tagged as originating from the hadronization of bottom quarks (b jets) using the combined secondary vertex (CSVv2) algorithm at its medium working point [66]. Additional $\mathrm{b}$ jet candidates are identified using the CSVv2 algorithm at its loose working point. The two working points, loose and medium, provide different levels of purity and efficiency. The loose working point gives a misidentification rate of approximately $10 \%$ for light-quark and gluon jets, with a b tagging efficiency of about $80 \%$. The medium working point has a misidentification rate of about $1 \%$ with a b tagging efficiency of about $68 \%$. The efficiency to tag c quarks is $12 \%$. To suppress the small residual QCD background, $p_{\mathrm{T}}^{\text {miss }}$ is required to be larger than 50 GeV. Studies on the estimation of non-prompt leptons from QCD multijet background by inverting lepton isolation selection criteria have verified that this background is negligible after applying the selection requirements. In addition, a requirement on the scalar sum of the $p_{\mathrm{T}}$ of all jets, $H_{\mathrm{T}}>500 \mathrm{GeV}$, is applied. The $H_{\mathrm{T}}$ requirement is used to suppress the $\mathrm{t} \overline{\mathrm{t}}$ background, while having little effect on the signal acceptance [20].

In the dilepton channels, a minimum of four jets is required, each with $|\eta|<2.4$. Of these, at least two must be b-tagged using the same CSVv2 algorithm with medium working point as was used in the single-lepton channel. While the $p_{\mathrm{T}}$ threshold for non-tagged jets is $30 \mathrm{GeV}$ (as for the single-lepton channel), the threshold for b-tagged jets is lowered to $25 \mathrm{GeV}$ to increase the acceptance for events with multiple b jets. The $H_{\mathrm{T}}>500 \mathrm{GeV}$ requirement is also applied to the dilepton channels.

Figures $2-5$ show the comparison of the data and simulations after these selections have been applied for both the single-lepton and dilepton analyses. The simulation of $t \bar{t}+$ jets process is split into three categories: top quark pair associated with two additional light flavor or gluon jets $(t \bar{t}+j j)$, top quark pair associated with a charm quark pair $(t \bar{t}+c \bar{c})$, and top quark pair associated with a bottom quark pair $(\mathrm{t} \overline{\mathrm{t}}+\mathrm{b} \overline{\mathrm{b}})$ [67]. The definitions of the variables in the figures are given in the next section.

\subsection{Multivariate discriminants}

Boosted decision trees (BDTs) $[68,69]$ are used in two roles in this analysis: to identify the top quarks and to improve the discrimination between signal and background. The jet multiplicity, jet properties and the number of the b jets, as well as associated kinematic variables, feature strongly in the choice of BDT input variables. The method is based on the strategies developed for the previous $13 \mathrm{TeV}$ CMS analyses in the single-lepton and opposite-sign dilepton final states [20]. All BDTs are trained using the ADABoost algorithm [70], as implemented in the TMVA package [71], and return a discriminant as output.

The BDT for identifying hadronically decaying top quarks classifies combinations of three jets (trijet) on how consistent they are with the trijet originating from the all-hadronic 
decay of a top quark, rather than from other sources such as initial-state radiation (ISR) or final-state radiation (FSR). Its input variables consist of the invariant dijet and trijet masses, the $b$ tagging information for the jet not associated to the dijet, and the angles between the three jets. This BDT is trained to distinguish between the three jets from a hadronically decaying top quark and any other permutation of 3-jet combinations using the ME information in $\mathrm{tt}+$ jets simulations.

Because of the high jet multiplicity in both signal and background events, many threejet combinations are possible. The trijet permutations for each event are ranked according to their discriminant value, from highest to lowest. In the single-lepton channel, each t $\overline{\mathrm{t}}$ background event contains a genuine hadronic top quark decay, so the jets included in the first-ranked trijet $\left(T_{\text {trijet1 }}\right)$ are removed and the highest-ranked discriminant using the remaining jets ( $\left.T_{\text {trijet2 }}\right)$ is used. In the dilepton channels, the t $\bar{t}$ background contains no hadronic top quark decays, so only the output for $T_{\text {trijet1 }}$ is used as the discriminant.

The BDTs, yielding the discriminants for the single-lepton channel $\left(D_{\mathrm{t} \overline{\mathrm{t}} \mathrm{t} \overline{\mathrm{t}}}^{\mathrm{SL}}\right)$ and for the dilepton channel $\left(D_{\mathrm{t} \overline{\mathrm{t}} \mathrm{t}}^{\mathrm{DL}}\right)$, use the discriminant from the trijet associations, described above, as one of its input variables. In the single-lepton channel, $D_{\mathrm{t} t \mathrm{t} \overline{\mathrm{t}}}^{\mathrm{SL}}$ is trained separately for each jet multiplicity, and inclusively over the number of b-tagged jets. In the dilepton channel, the training is done unitarily for all jet multiplicities while separately in $\mu^{+} \mu^{-}, \mu^{ \pm} \mathrm{e}^{\mp}$, and $\mathrm{e}^{+} \mathrm{e}^{-}$states. The choice of input variables is optimized separately for the two channels and is based on the characteristics of the lepton and jet activity in the events. The resulting variable lists are different for the two channels. The variables can be grouped into three categories: event activity, event topology, and b quark multiplicity. Although many of the input variables are correlated, each one contributes some additional discrimination between the $t \bar{t}$ background and the $t \bar{t} t \bar{t}$ signal.

Studies of the differences between the simulated $t \bar{t}$ and $t \bar{t} t \bar{t}$ events have led to the selection of the following variables describing the hadronic activity in the event:

1. The number of jets present in the event, $N_{\mathrm{j}}$.

2. The scalar sum of the $p_{\mathrm{T}}$ of all medium working point $\mathrm{b}$ jets in the event, $H_{\mathrm{T}}^{\mathrm{b}}$.

3. The ratio of the sum $H_{\mathrm{T}}$ of the four highest $p_{\mathrm{T}}$ jets in the event in the single-lepton channel, or the two jets with the highest $\mathrm{b}$ tagging discriminant in the dilepton channel, to the $H_{\mathrm{T}}$ of the other jets in the event, $H_{\mathrm{T}}^{\text {ratio }}$.

4. The $H_{\mathrm{T}}$ sum in the event, subtracting the scalar $p_{\mathrm{T}}$ sum of the two highest $p_{\mathrm{T}} \mathrm{b}$ jets, $H_{\mathrm{T}}^{2 \mathrm{~m}}$.

5. The transverse momenta of the jets with the third- and fourth-largest $p_{\mathrm{T}}$ in the event, $p_{\mathrm{T}}^{\mathrm{j} 3}$ and $p_{\mathrm{T}}^{\mathrm{j} 4}$.

6. The reduced event mass, $M_{\text {red }}^{\mathrm{h}}$, defined as the invariant mass of the system comprising all the jets in the reduced event, where the reduced event is constructed by removing the jets contained in $T_{\text {trijet } 1}$ in single-lepton events. In $t \bar{t}$ events, the reduced event will typically only contain the b jet from the semileptonic top quark decay and jets 


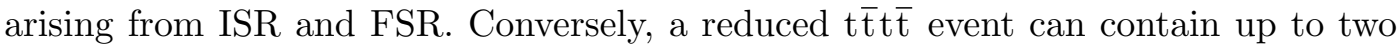
hadronically decaying top quarks and, as a result, a relatively high reduced event mass.

7. The reduced event $H_{\mathrm{T}}, H_{\mathrm{T}}^{\mathrm{x}}$, is defined as the $H_{\mathrm{T}}$ of all jets in the single-lepton event selection excluding those contained in $T_{\text {trijet1 }}$.

The event topology is characterized by the two variables:

1. Event sphericity, $S$, [72], calculated from all of the jets in the event in terms of the normalized tensor $M^{\alpha \beta}=\sum_{i} p_{i}^{\alpha} p_{i}^{\beta} / \sum_{i}\left|\overrightarrow{p_{i}}\right|^{2}$, where $\alpha$ and $\beta$ refer to the three-components of the momentum of the $i$ th jet. The sphericity is defined as $S=(3 / 2)\left(\lambda_{2}+\lambda_{3}\right)$, where $\lambda_{2}$ and $\lambda_{3}$ are the two smallest eigenvalues of $M^{\alpha \beta}$. The sphericity in $\mathrm{t} \overline{\mathrm{t}} \mathrm{t} \overline{\mathrm{t}}$ events should differ from that in background t $\bar{t}$ events of the same energy, since the jets in $t \bar{t}$ events will be less isotropically distributed because of their recoil from sources such as ISR.

2. Hadronic centrality, $C$, defined as the value of $H_{\mathrm{T}}$ divided by the sum of the energies of all jets in the event.

Since all these variables rely only on the hadronic information in the event, sensitivity to the lepton information is provided through the $p_{\mathrm{T}}$ and $\eta$ of the highest $p_{\mathrm{T}}$ lepton (or the only lepton for the single-lepton channel) $\left(p_{\mathrm{T}}^{\ell 1}, \eta^{\ell 1}\right)$ and the angular difference $\left(\Delta R_{\ell \ell}\right)$ between the leptons in dilepton events. The $\mathrm{b}$ jet multiplicity is characterized in terms of the number of b jets tagged by the CSVv2 algorithm operating at its loose $\left(N_{\text {tags }}^{1}\right)$ and medium $\left(N_{\text {tags }}^{\mathrm{m}}\right)$ operating points, and the angular separation $\Delta R_{\mathrm{bb}}$ between the b-tagged jets with the highest CSVv2 discriminants. Finally, the third- and fourth-highest $b$ tagging discriminant values are used as they allow separation between $t \bar{t}+$ light jets, and genuine additional heavy-flavor jets, as present in $t \bar{t} t \bar{t}$ events.

The training variables were not changed as a function of final state or jet multiplicity. In the single-lepton channel, the optimal variable set, listed in the order of their discriminating power, is $T_{\text {trijet } 2} ; H_{\mathrm{T}}^{\mathrm{b}} ; C ; p_{\mathrm{T}}^{\ell 1} ; M_{\mathrm{red}}^{\mathrm{h}} ; H_{\mathrm{T}}^{\mathrm{x}}$; the third- and fourth-highest CSVv2 discriminants, and the $p_{\mathrm{T}}$ of those tagged jets; the $p_{\mathrm{T}}$ for the first, second, fifth, and sixth jet. In the dilepton channel, the optimal variable set, listed in the order of their discriminating power, is $N_{\mathrm{j}}, T_{\text {trijet } 1}, H_{\mathrm{T}}^{2 \mathrm{~m}}, p_{\mathrm{T}}^{\mathrm{j} 4}, N_{\text {tags }}^{1}, H_{\mathrm{T}}^{\text {ratio }}, H_{\mathrm{T}}^{\mathrm{b}}, S, \Delta R_{\mathrm{bb}}, N_{\text {tags }}^{\mathrm{m}}, \Delta R_{\ell \ell}, C, p_{\mathrm{T}}^{\mathrm{j} 3}, p_{\mathrm{T}}^{\ell 1}$ and $\eta^{\ell 1}$. The $\mathrm{MC}$ modeling of the individual observables utilized in the discriminants $D_{\mathrm{t} \overline{\mathrm{t}} \overline{\mathrm{t}}}^{\mathrm{SL}}$ and $D_{\mathrm{t} \mathrm{t} \mathrm{t}}^{\mathrm{DL}}$ was verified using samples of $t \bar{t}$ events and found to be in agreement with the data for all the jet and $b$ jet multiplicities.

\section{Systematic uncertainties}

The systematic uncertainties that affect this analysis can change the shape, or the normalization, or both, of the $D_{\mathrm{t} \overline{\mathrm{t}} \overline{\mathrm{t}}}^{\mathrm{SL}}$ and $D_{\mathrm{t} \overline{\mathrm{t}} \overline{\mathrm{t}}}^{\mathrm{DL}}$ discriminants. The uncertainties are characterized in table 1. Each of the systematic uncertainty sources is modeled by one nuisance parameter. The normalization-dependent terms account for the uncertainties in the background 

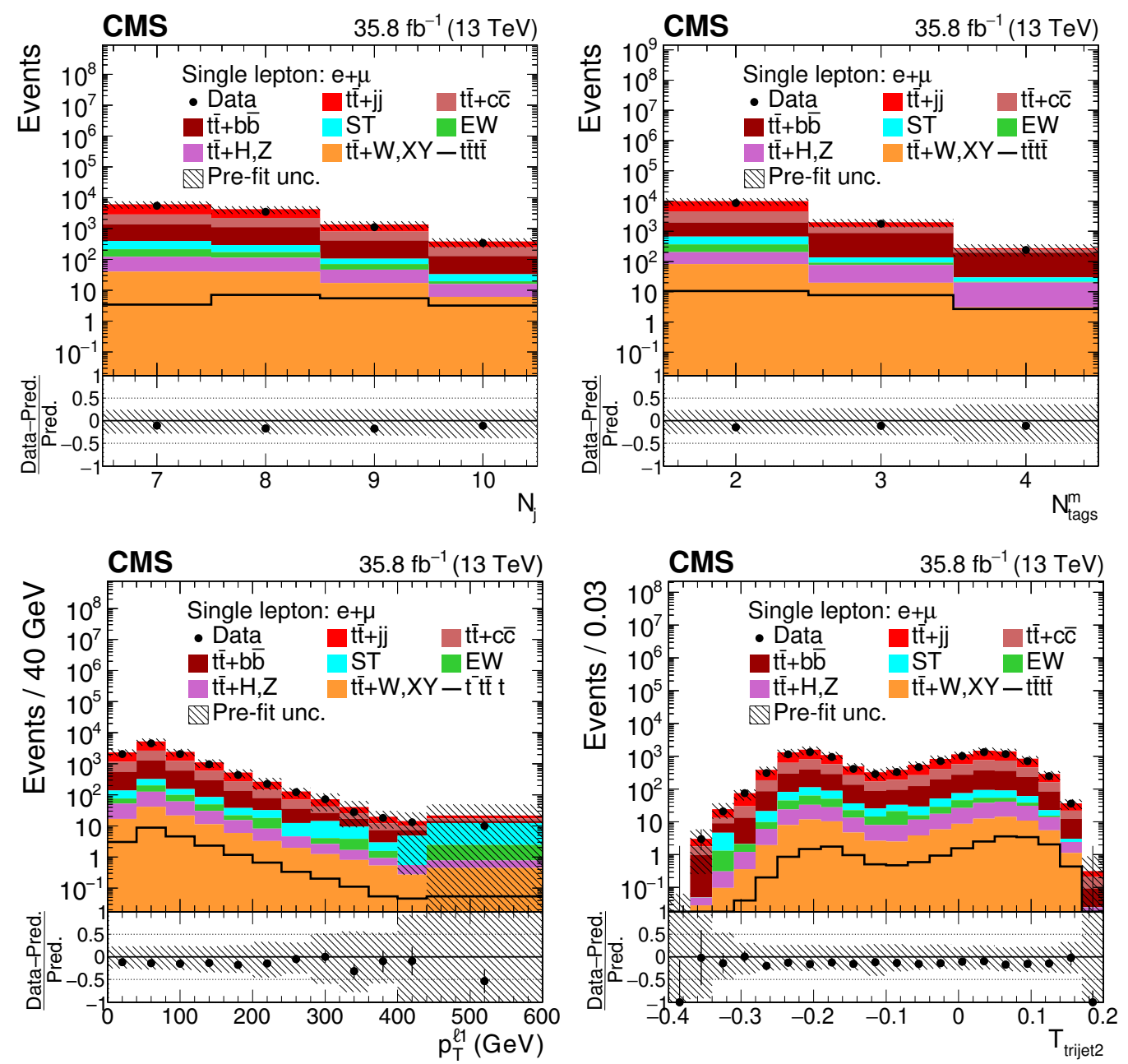

Figure 2. Distributions of $N_{\mathrm{j}}, N_{\mathrm{tags}}^{\mathrm{m}}, p_{\mathrm{T}}^{\ell 1}$ and $T_{\text {trijet2 }}$ in the combined single-lepton channels. In the upper panels, the data are shown as dots with error bars representing statistical uncertainties, MC simulations are shown as a histogram. The lower panels show the relative difference between the data and the sum of all of the standard model backgrounds. In each panel, the shaded band represents the total uncertainty in the dominant $t \bar{t}$ background estimate. See section 4.2 for the definitions of the variables.

yields, while the effect of the shape-dependent terms is evaluated using discriminant distributions whose shape has been modified by each of the uncertainties.

The experimental uncertainties considered are:

- Integrated luminosity: a $2.5 \%$ normalization uncertainty on the integrated luminosity [73].

- Pileup modeling: the number of pileup events in the simulation is matched to that of the data. The uncertainty due to this correction is estimated by using two sets of alternative weights derived with a variation of $\pm 4.6 \%$ on the total inelastic pp cross section [74]. 

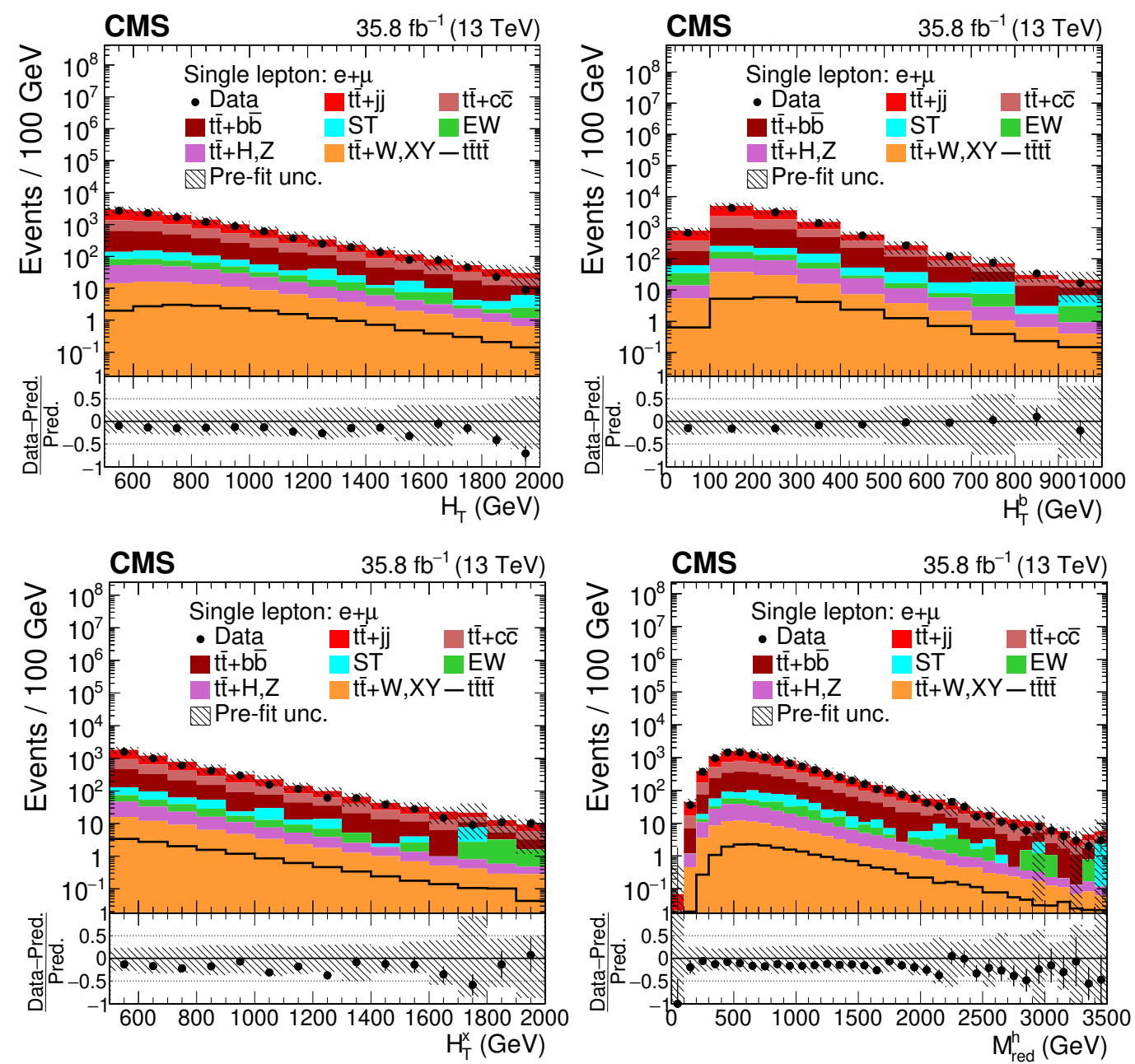

Figure 3. Distributions of $H_{\mathrm{T}}, H_{\mathrm{T}}^{\mathrm{b}}, H_{\mathrm{T}}^{\mathrm{x}}$ and $M_{\mathrm{red}}^{\mathrm{h}}$ in the combined single-lepton channel. In the upper panels, the data are shown as dots with error bars representing statistical uncertainties, MC simulations are shown as a histogram. The lower panels show the relative difference between the data and the sum of all of the standard model backgrounds. In each panel, the shaded band represents the total uncertainty in the dominant $t \bar{t}$ background estimate. See section 4.2 for the definitions of the variables.

- Lepton reconstruction and identification: the uncertainties in lepton identification, isolation, trigger efficiencies, and tracking efficiencies were examined. After a comparison between data and simulations, we assign a normalization uncertainty of $3 \%$ to take into account these effects.

- Jet energy corrections and resolutions: the uncertainties due to limited knowledge of the jet energy scale (JES) and the jet energy resolution (JER) are estimated by varying the $\eta$ - and $p_{\mathrm{T}}$-dependent JES and JER corrections of all jets by \pm 1 standard deviation [64]. In the case of JES uncertainty, it was split into 6 components, which include uncertainties owing to the absolute jet-energy scale, the pileup offset, the extrapolation between samples of different jet-flavor composition, the parton 

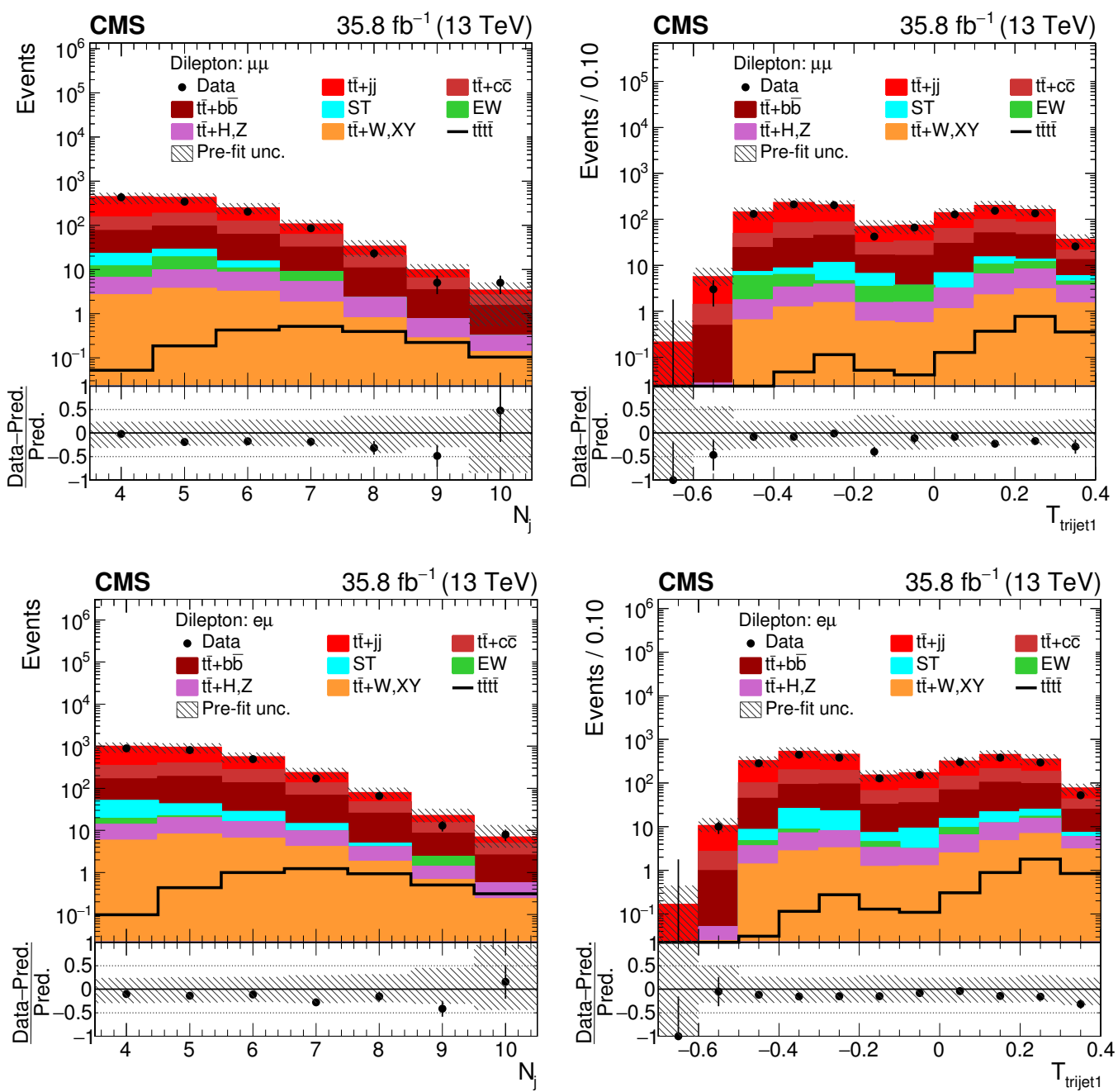

Figure 4. Distributions of $N_{\mathrm{j}}$ and $T_{\text {trijet1 }}$ in the $\mu^{+} \mu^{-}$(upper row) and $\mu^{ \pm} \mathrm{e}^{\mp}$ (lower row) channels. In the upper panels of each figure, the data are shown as dots with error bars representing statistical uncertainties, MC simulations are shown as a histogram. The lower panels show the relative difference between the data and the sum of all of the standard model backgrounds. In each panel, the shaded band represents the total uncertainty in the dominant t $\bar{t}$ background estimate. See section 4.2 for the definitions of the variables.

fragmentation and underlying event modeling and residual time and $\eta$-dependent variations. Each component represents a quadratic sum of subsets of jet energy correction uncertainties from different sources. The effect of each component is evaluated separately.

- $b$ tagging: the uncertainty in the $b$ tagging discriminant shape is estimated by varying the shape of the discriminant distribution according to its one standard deviation uncertainties in terms of the $p_{\mathrm{T}}, \eta$, and flavor of the jets [67]. The variations correspond to uncertainties in the jet energy scale, background contamination of the samples used to derive them, and statistical uncertainties of these data samples. 

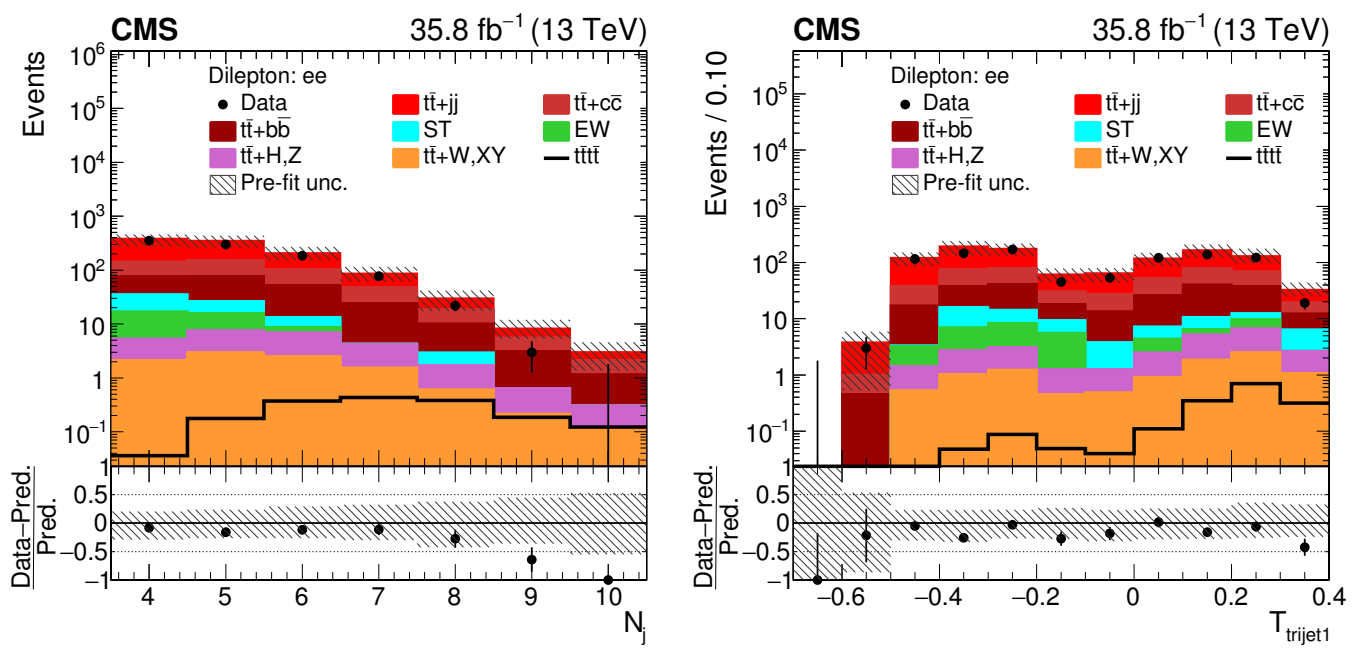

Figure 5. Distributions of $N_{\mathrm{j}}$ and $T_{\text {trijet1 }}$ in the $\mathrm{e}^{+} \mathrm{e}^{-}$channel. In the upper panels of each figure, the data are shown as dots with error bars representing statistical uncertainties, MC simulations are shown as a histogram. The lower panels show the relative difference between the data and the sum of all of the standard model backgrounds. In each panel, the shaded band represents the total uncertainty in the dominant $t \bar{t}$ background estimate. See section 4.2 for the definitions of the variables.

\begin{tabular}{|lcc|}
\hline Systematic uncertainty & Normalization & Shape \\
\hline Integrated luminosity & $\mathrm{X}$ & \\
Pileup modeling & $\mathrm{X}$ & $\mathrm{X}$ \\
Lepton reconstruction and identification & $\mathrm{X}$ & \\
Jet energy corrections & $\mathrm{X}$ & $\mathrm{X}$ \\
b tagging & $\mathrm{X}$ & $\mathrm{X}$ \\
Ren. and fact. scales & $\mathrm{X}$ & $\mathrm{X}$ \\
PS scales & $\mathrm{X}$ & \\
ME-PS matching & $\mathrm{X}$ & \\
UE & $\mathrm{X}$ & \\
Jet multiplicity correction & $\mathrm{X}$ & \\
Parton distribution functions & $\mathrm{X}$ & $\mathrm{X}$ \\
Top quark $p_{\mathrm{T}}$ reweighting & & $\mathrm{X}$ \\
Heavy-flavor reweighting & $\mathrm{X}$ & $\mathrm{X}$ \\
Rare process & $\mathrm{X}$ & \\
\hline
\end{tabular}

Table 1. Uncertainties that affect the normalization of the data sets and shapes of the $D_{\mathrm{t} \bar{t} \mathrm{t}}^{\mathrm{SL}}$ and $D_{\mathrm{t} \overline{\mathrm{t}} \mathrm{t} \overline{\mathrm{E}}}^{\mathrm{DL}}$ discriminants. Their contribution to different effects are marked by X. 
Sources of systematic uncertainties originating from theory are listed below.

- Renormalization and factorization scales: in order to estimate the uncertainty arising from missing higher-order terms in the calculation of the signal and background cross sections, renormalization and factorization scales are each modified, independently, up and down by a factor of two relative to their nominal values. The cases in which the two scales are varied in opposite directions are excluded. This is estimated for both the $t \bar{t}$ and $t \bar{t} t \bar{t}$ processes.

- Parton shower scales: the evolution scales in the initial- and final-state PSs are separately varied by a factor of 2 and $\sqrt{2}$, respectively, up and down relative to their nominal values, in order to estimate the uncertainty attributed to the shower model. This is estimated for both the $t \bar{t}$ and $t \bar{t} t \bar{t}$ processes.

- ME-PS matching: the uncertainty resulting from this source is estimated by varying the POWHEG-BOX PS scale parameter, $h_{\text {damp }}$, that controls the ME and PS matching and regulates the high- $p_{\mathrm{T}}$ radiation, within its uncertainty by \pm 1 standard deviation of the measured value $h_{\text {damp }}=1.581_{-0.585}^{+0.658} m_{\mathrm{t}}$ [54]. This is estimated for the $\mathrm{t} \overline{\mathrm{t}}$ process.

- Underlying event: the uncertainty from the UE tune of t $\overline{\mathrm{t}}$ event generator is evaluated by using simulations with varied parameters that are related to the CUETP8M2T4

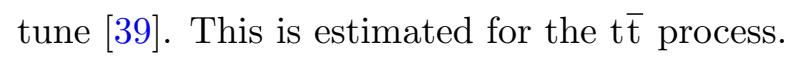

- Jet multiplicity correction: the modeling of $t \bar{t}+$ jets production in POWHEG-BOX is insufficient to describe the data in the regions of large jet multiplicity. To allow for this, scale factors are determined from fits to the single-lepton data in the signal depleted regions $\left(N_{\mathrm{j}}=8,9\right.$, and $\left.N_{\text {tags }}^{\mathrm{m}}=2,3\right)$, and propagated to the signal sensitive regions. The scale factors determined in the single-lepton channel are also used in the dilepton channel taking into account the difference in the jet multiplicity between the two channels. The uncertainty resulting from this correction is $10 \%$ for the $t \bar{t}$ process [53].

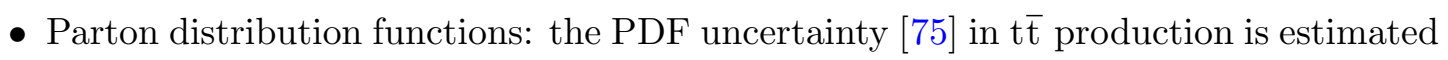
by evaluating the shape difference between the nominal simulation and simulations based on the NNPDF [50], MMHT14 [76], and CT10 [77] PDF sets. This is estimated for the $t \bar{t}$ process.

- Top quark $p_{\mathrm{T}}$ reweighting: the $\mathrm{t} \overline{\mathrm{t}}$ simulation is corrected to match the observed spectra $[78,79]$. The uncertainty from the corrections made to the shape of the top quark $p_{\mathrm{T}}$ distribution is estimated by allowing the correction function to vary within $\mathrm{a} \pm 1$ standard deviation uncertainty. This is estimated for the t $\overline{\mathrm{t}}$ process.

- Heavy-flavor reweighting: to correctly model the rate of additional heavy-flavor jets in $t \bar{t}$ production, the uncertainty in the rate of $t \bar{t}+b \bar{b}$ is taken from the \pm 1 standard deviation uncertainty in the measured value [80]. This is estimated for the $t \bar{t}$ process. 
As a cross-check, an independent uncertainty on $t \bar{t}+c \bar{c}$ production was added. The resulting effect on the expected sensitivity of the search was found to be negligible.

- Rare processes: uncertainties from the cross sections of rare processes of $t \bar{t}$ pair production in association with one or two massive gauge bosons and triple top quark production are taken into account by allowing them to vary within $50 \%$ of their SM value $[21]$.

The simulated samples used to evaluate the PS, ME-PS and UE uncertainties are statistically limited, so these uncertainties are estimated conservatively by assigning the larger value between the statistical uncertainty of these simulated samples and the rate change of these simulated sample from the nominal simulation as uncertainty, independently for different jet multiplicities.

\section{Results}

A simultaneous binned maximum-likelihood template fit to the single-lepton, dilepton, and combined experimental results was used to determine the signal strength parameter, which is defined as the ratio of the observed and predicted SM t $\overline{\mathrm{t}} \mathrm{t} \overline{\mathrm{t}}$ cross sections, $\mu=\sigma_{\mathrm{t} \mathrm{t} \overline{\mathrm{t}}}^{\mathrm{obs}} / \sigma_{\mathrm{t} \overline{\mathrm{t}} \mathrm{t} \overline{\mathrm{t}}}^{\mathrm{SM}}$. To increase the sensitivity of the analysis, events are categorized depending on their jet and b-tagged jet multiplicities. In the single-lepton channel these categories are: $N_{\mathrm{j}}=7$, 8,9 , and $\geq 10$ and $N_{\text {tags }}^{\mathrm{m}}=2,3$, and $\geq 4$ in each jet multiplicity region. In the dilepton channel these are $N_{\mathrm{j}}=4-5,6-7$, and $\geq 8$ and $N_{\text {tags }}^{\mathrm{m}}=2$, and $\geq 3$ in each jet multiplicity region. In each category the binning was chosen to ensure at least 4 predicted background events per bin.

The likelihood function incorporates each of the systematic uncertainties in the signal and background $D_{\mathrm{t} \overline{\mathrm{t}} \overline{\mathrm{t}}}^{\mathrm{DL}}$ and $D_{\mathrm{t} \overline{\mathrm{t}} \overline{\mathrm{t}}}^{\mathrm{SL}}$ templates as nuisance parameters in the fit. The systematic uncertainties attributed to the trigger or specific to the jet or lepton reconstruction were treated as fully correlated among the different final states. The normalization uncertainties are included assuming a log-normal distribution for the nuisance parameters, while the shape uncertainties are included as Gaussian-distributed parameters.

All of the post-fit nuisance parameter values were found to be consistent with their initial values to well within their quoted uncertainties, indicating the consistency of the fit model with the observed data. Two of the post-fit nuisance parameters are significantly constrained by the fit. These correspond to the heavy-flavor reweighting and initial-state parton-shower radiation scale, which are reduced by $65 \%$ and $30 \%$, respectively. The sensitivity of the analysis is affected almost equally by the statistical uncertainty and the combined systematic uncertainties. The leading sources of systematic uncertainty are the $\mathrm{t} \overline{\mathrm{t}}+$ heavy-flavor production reweighting, the jet multiplicity correction, and the PS and UE modeling in t $\overline{\mathrm{t}}$ simulation. The post-fit distributions in signal-enriched $N_{\mathrm{j}}$ and $N_{\mathrm{tags}}^{\mathrm{m}}$ categories are shown in figures 6-9 for the single-lepton channel and figures 11-12 for the dilepton channel.

No statistically significant deviation from the SM background prediction is observed in the $D_{\mathrm{t} \overline{\mathrm{t}} \overline{\mathrm{t}}}^{\mathrm{DL}}$ or $D_{\mathrm{t} \mathrm{t} t \overline{\mathrm{t}}}^{\mathrm{SL}}$ distributions. The corresponding observed and expected significance 


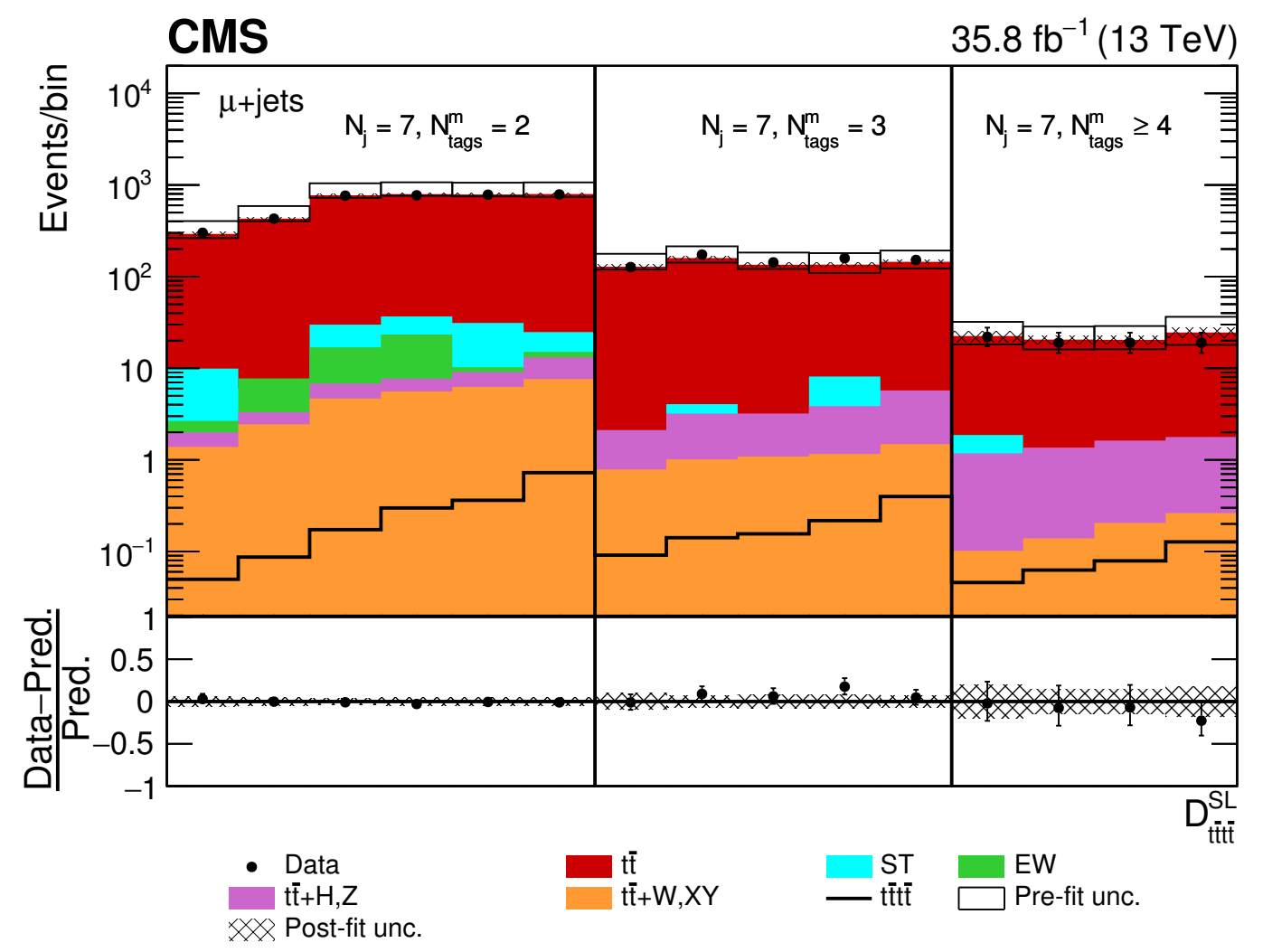

Figure 6. Post-fit $D_{\mathrm{t} \mathrm{t} t \overline{\mathrm{t}}}^{\mathrm{SL}}$ distribution in the single-muon channel for events satisfying baseline singlelepton selection and $N_{\mathrm{j}}=7, N_{\mathrm{tags}}^{\mathrm{m}}=2,3, \geq 4$. Non-uniform binning of the BDT discriminant was

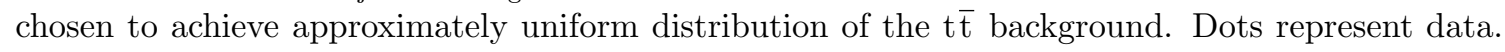
Vertical error bars show the statistical uncertainties in data. The post-fit background predictions are shown as shaded histograms. Open boxes demonstrate the size of the pre-fit uncertainty in the total background and are centered around the pre-fit expectation value of the prediction. The hatched area shows the size of the post-fit uncertainty in the background prediction. The signal histogram template is shown as a solid line. The lower panel shows the relative difference of the observed number of events over the post-fit background prediction.

of the signal and the best fit value of the signal strength parameter are given together with the $t \bar{t} t \bar{t}$ cross section in table 2. In order to quantify the experimental sensitivity of the search, the median expected significance is calculated assuming that the data are distributed according to the SM prediction with a nominal t $\overline{\mathrm{t}} \mathrm{t} \overline{\mathrm{t}}$ production cross section value $\sigma_{\mathrm{t} \overline{\mathrm{t}} \mathrm{t}}^{\mathrm{SM}}$, corresponding to the signal strength modifier value $\mu=1$. An upper limit on the $t \bar{t} t \bar{t}$ production cross section is derived using the asymptotic approximation of the $\mathrm{CL}_{\mathrm{s}}$ method [81-85]. The observed and expected 95\% confidence level (CL) upper limits from the two analyses and their combination are listed in table 3. The expected upper limit on the $t \bar{t} t \bar{t}$ production is calculated under assumption of a background-only hypothesis, corresponding to the signal strength modifier $\mu=0$. 

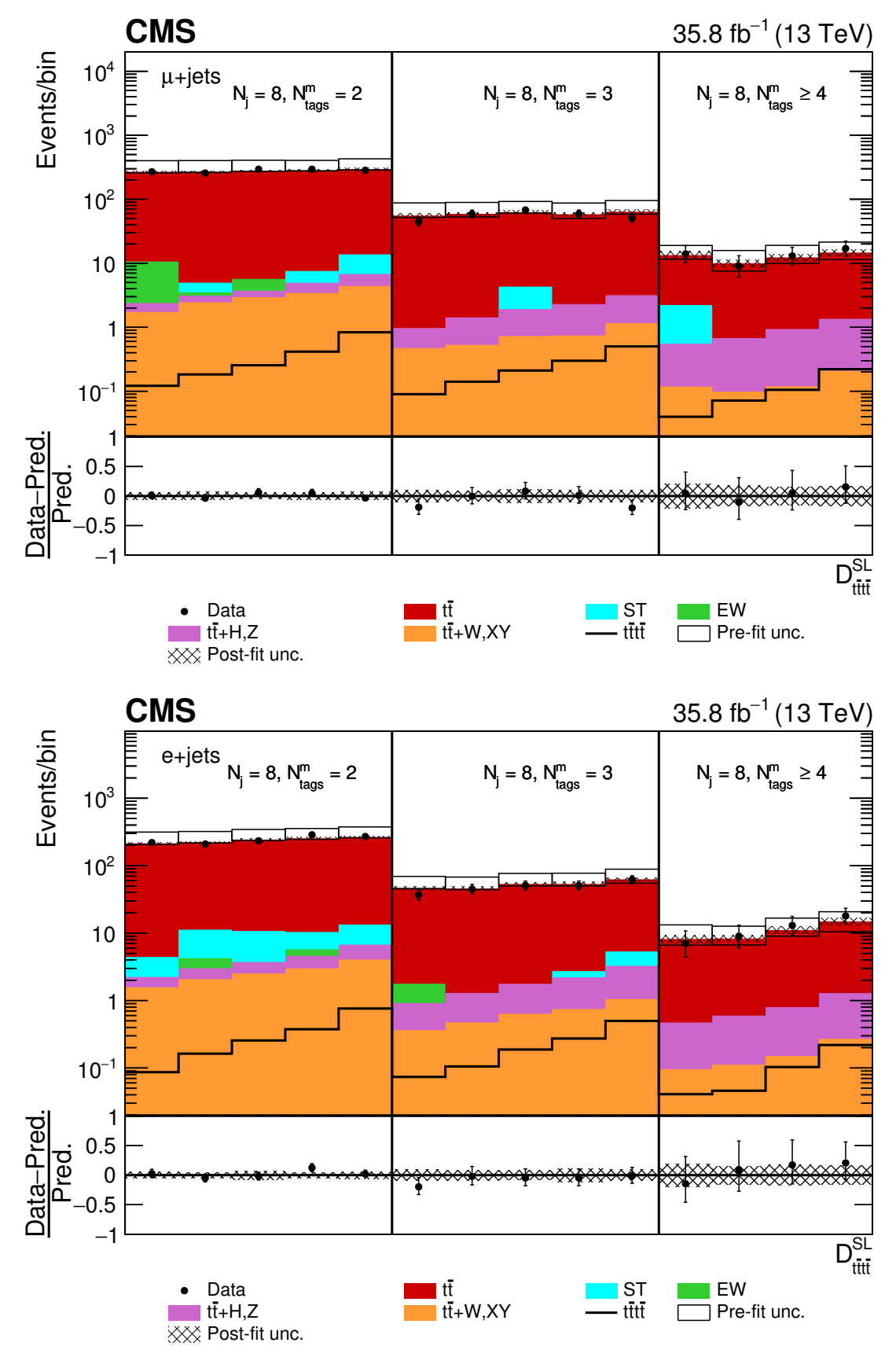

Figure 7. Post-fit $D_{\mathrm{t} t \mathrm{t} \overline{\mathrm{t}}}^{\mathrm{SL}}$ distribution in the (upper row) single-muon and (lower row) singleelectron channels for events satisfying baseline single-lepton selection and $N_{\mathrm{j}}=8, N_{\text {tags }}^{\mathrm{m}}=2,3$, $\geq 4$. Non-uniform binning of the BDT discriminant was chosen to achieve approximately uniform distribution of the $t \bar{t}$ background. Dots represent data. Vertical error bars show the statistical uncertainties in data. The post-fit background predictions are shown as shaded histograms. Open boxes demonstrate the size of the pre-fit uncertainty in the total background and are centered around the pre-fit expectation value of the prediction. The hatched area shows the size of the post-fit uncertainty in the background prediction. The signal histogram template is shown as a solid line. The lower panel shows the relative difference of the observed number of events over the post-fit background prediction. 

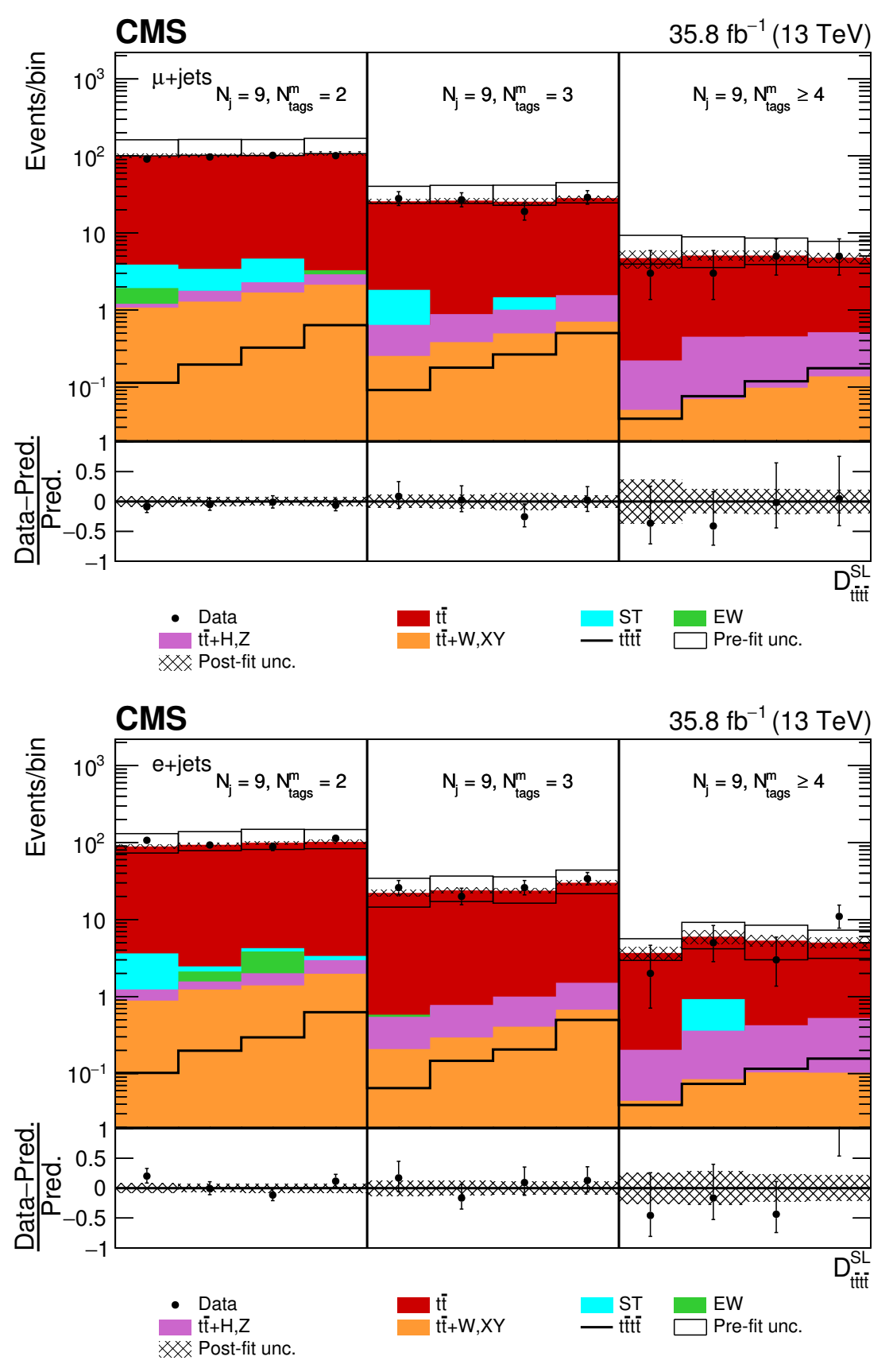

Figure 8. Post-fit $D_{\mathrm{t} \overline{\mathrm{t} t} \overline{\mathrm{t}}}^{\mathrm{SL}}$ distribution in the (upper row) single-muon and (lower row) singleelectron channels for events satisfying baseline single-lepton selection and $N_{\mathrm{j}}=9, N_{\text {tags }}^{\mathrm{m}}=2,3$, $\geq 4$. Non-uniform binning of the BDT discriminant was chosen to achieve approximately uniform distribution of the $t \bar{t}$ background. Dots represent data. Vertical error bars show the statistical uncertainties in data. The post-fit background predictions are shown as shaded histograms. Open boxes demonstrate the size of the pre-fit uncertainty in the total background and are centered around the pre-fit expectation value of the prediction. The hatched area shows the size of the post-fit uncertainty in the background prediction. The signal histogram template is shown as a solid line. The lower panel shows the relative difference of the observed number of events over the post-fit background prediction. 

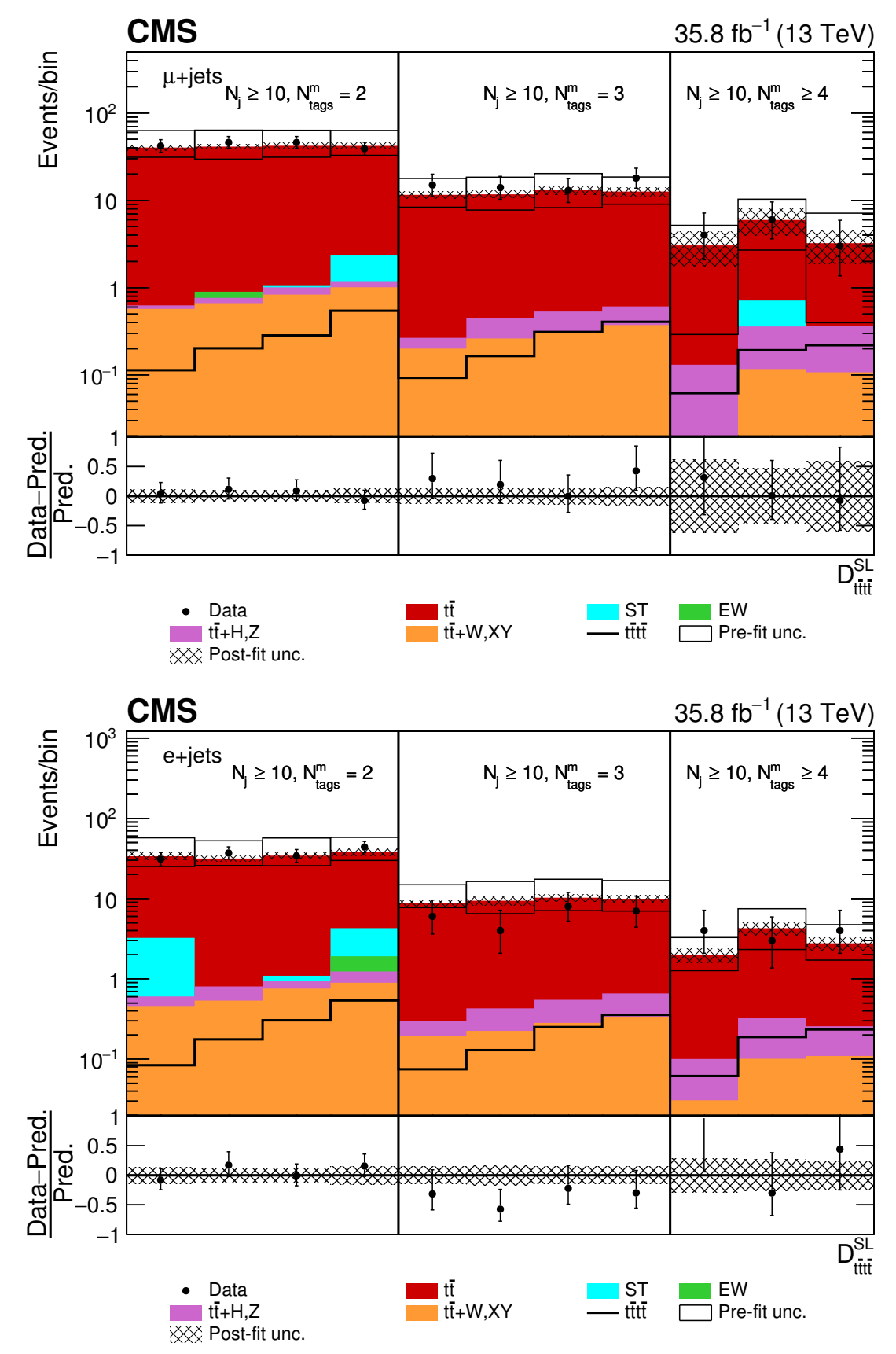

Figure 9. Post-fit $D_{\mathrm{t} t \mathrm{t} \overline{\mathrm{t}}}^{\mathrm{SL}}$ distribution in the (upper row) single-muon and (lower row) singleelectron channels for events satisfying baseline single-lepton selection and $N_{\mathrm{j}} \geq 10, N_{\text {tags }}^{\mathrm{m}}=2,3$, $\geq 4$. Non-uniform binning of the BDT discriminant was chosen to achieve approximately uniform distribution of the $t \bar{t}$ background. Dots represent data. Vertical error bars show the statistical uncertainties in data. The post-fit background predictions are shown as shaded histograms. Open boxes demonstrate the size of the pre-fit uncertainty in the total background and are centered around the pre-fit expectation value of the prediction. The hatched area shows the size of the post-fit uncertainty in the background prediction. The signal histogram template is shown as a solid line. The lower panel shows the relative difference of the observed number of events over the post-fit background prediction. 

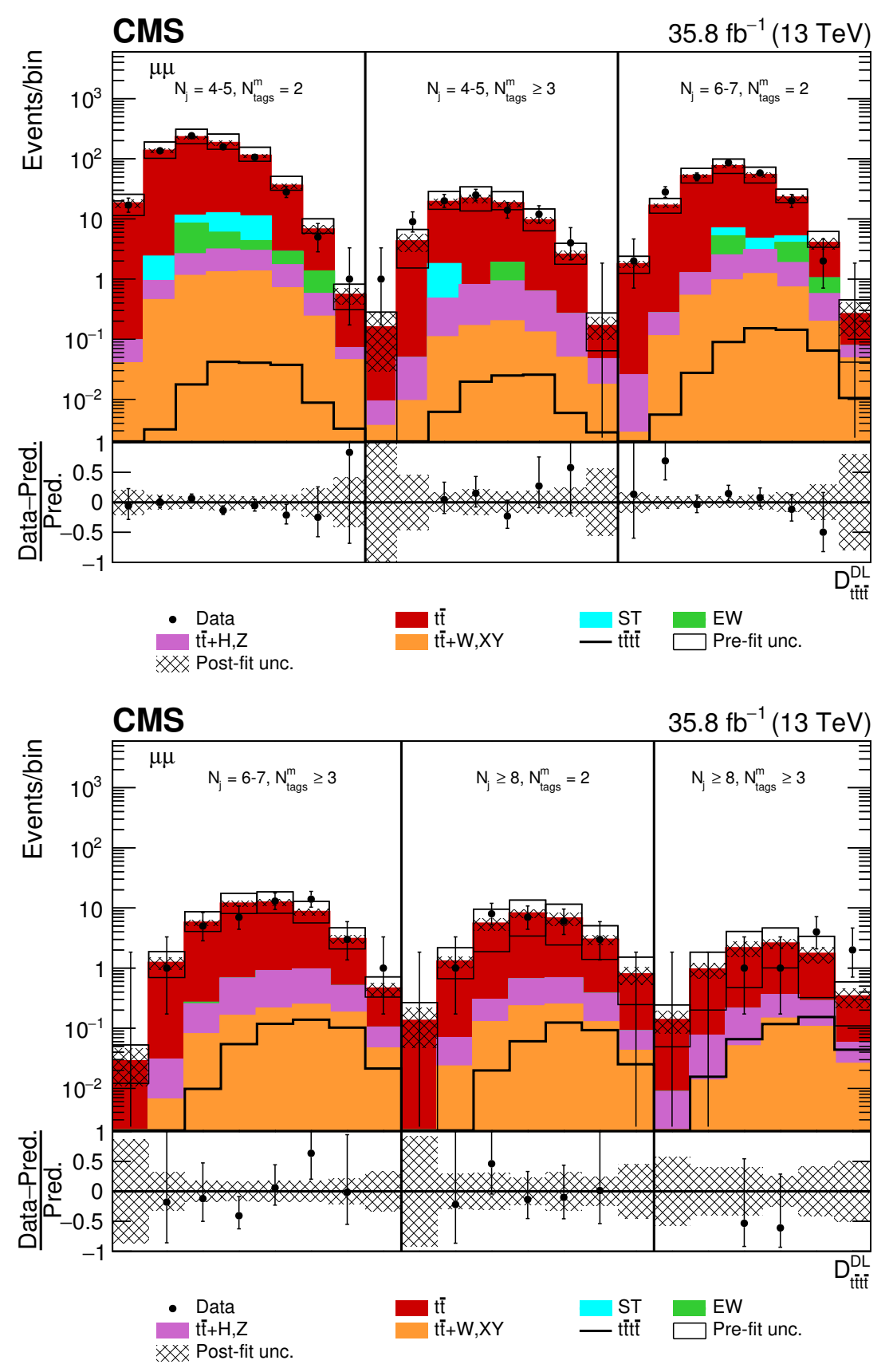

Figure 10. Post-fit $D_{\mathrm{t} \overline{\mathrm{t}} \overline{\mathrm{t}} \mathrm{D}}^{\mathrm{L}}$ distributions in the $\mu^{+} \mu^{-}$channel for events satisfying baseline oppositesign dilepton selection and (upper row) $N_{\mathrm{j}}=4-5, N_{\text {tags }}^{\mathrm{m}}=2, \geq 3, N_{\mathrm{j}}=6-7, N_{\text {tags }}^{\mathrm{m}}=2$ and (lower row) $N_{\mathrm{j}}=6-7, N_{\text {tags }}^{\mathrm{m}} \geq 3, N_{\mathrm{j}} \geq 8, N_{\text {tags }}^{\mathrm{m}}=2, \geq 3$. Dots represent data. Vertical error bars show the statistical uncertainties in data. The post-fit background predictions are shown as shaded histograms. Open boxes demonstrate the size of the pre-fit uncertainty in the total background and are centered around the pre-fit expectation value of the prediction. The hatched area shows the size of the post-fit uncertainty in the background prediction. The signal histogram template is shown as a solid line. The lower panel shows the relative difference of the observed number of events over the post-fit background prediction. 

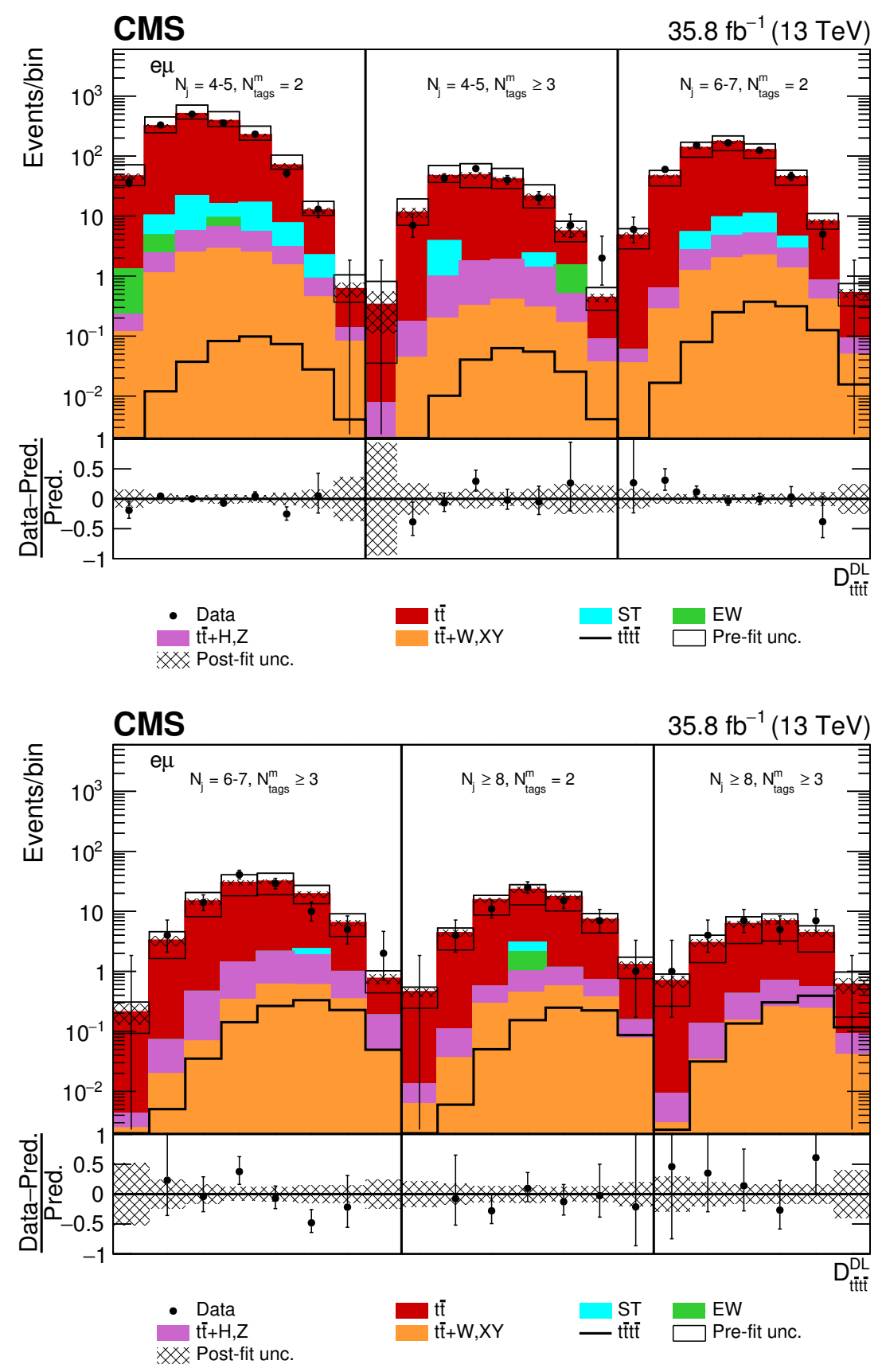

Figure 11. Post-fit $D_{\mathrm{t} t \mathrm{t}}^{\mathrm{DL}}$ distributions in the $\mu^{ \pm} \mathrm{e}^{\mp}$ channel for events satisfying baseline oppositesign dilepton selection and (upper row) $N_{\mathrm{j}}=4-5, N_{\mathrm{tags}}^{\mathrm{m}}=2, \geq 3, N_{\mathrm{j}}=6-7, N_{\mathrm{tags}}^{\mathrm{m}}=2$ and (lower row) $N_{\mathrm{j}}=6-7, N_{\text {tags }}^{\mathrm{m}} \geq 3, N_{\mathrm{j}} \geq 8, N_{\text {tags }}^{\mathrm{m}}=2, \geq 3$. Dots represent data. Vertical error bars show the statistical uncertainties in data. The post-fit background predictions are shown as shaded histograms. Open boxes demonstrate the size of the pre-fit uncertainty in the total background and are centered around the pre-fit expectation value of the prediction. The hatched area shows the size of the post-fit uncertainty in the background prediction. The signal histogram template is shown as a solid line. The lower panel shows the relative difference of the observed number of events over the post-fit background prediction. 

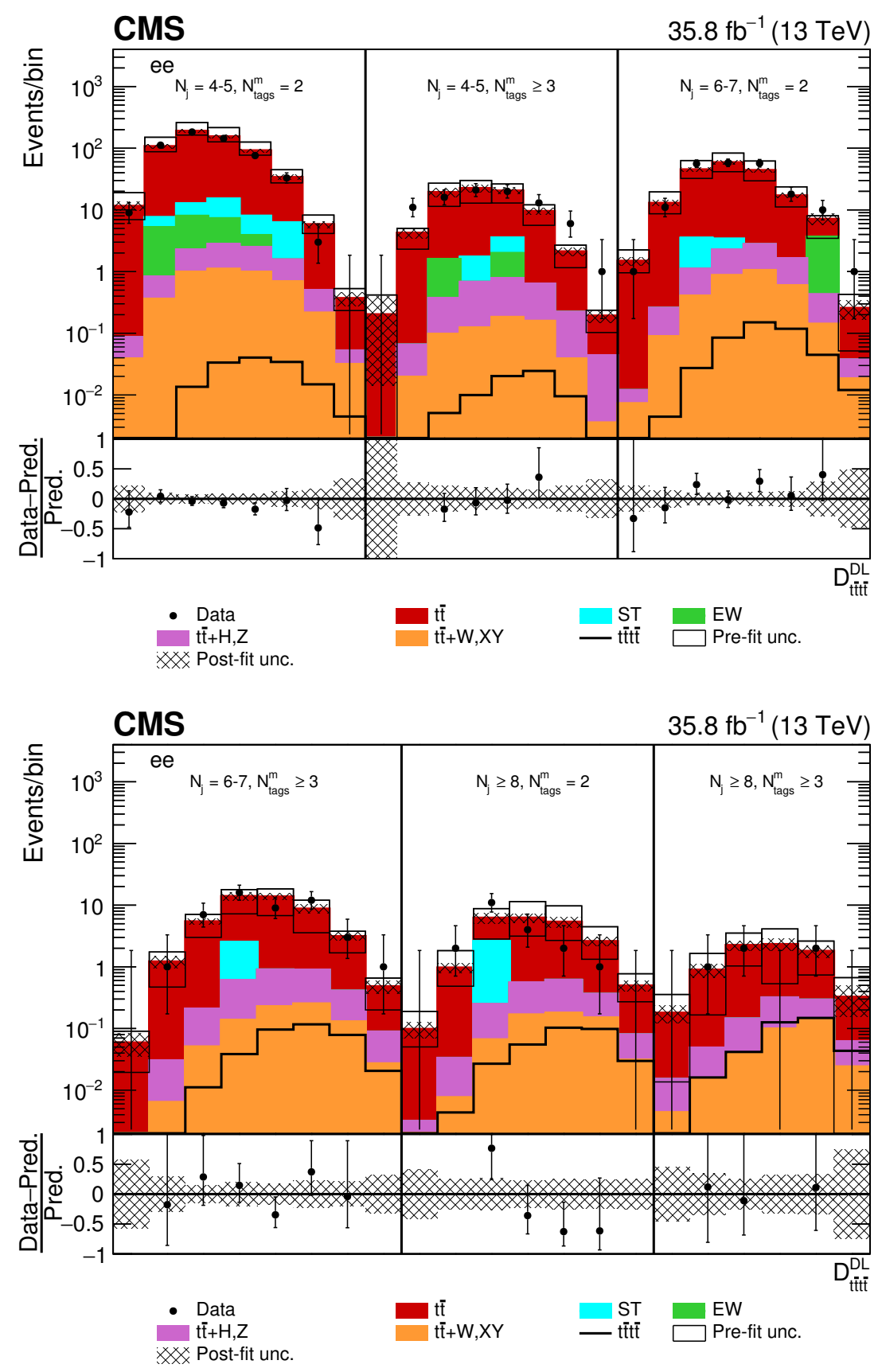

Figure 12. Post-fit $D_{\mathrm{tt} t \overline{\mathrm{t}}}^{\mathrm{DL}}$ distributions in the $\mathrm{e}^{+} \mathrm{e}^{-}$channel for events satisfying baseline oppositesign dilepton selection and (upper row) $N_{\mathrm{j}}=4-5, N_{\mathrm{tags}}^{\mathrm{m}}=2, \geq 3, N_{\mathrm{j}}=6-7, N_{\mathrm{tags}}^{\mathrm{m}}=2$ and (lower row) $N_{\mathrm{j}}=6-7, N_{\text {tags }}^{\mathrm{m}} \geq 3, N_{\mathrm{j}} \geq 8, N_{\text {tags }}^{\mathrm{m}}=2, \geq 3$. Dots represent data. Vertical error bars show the statistical uncertainties in data. The post-fit background predictions are shown as shaded histograms. Open boxes demonstrate the size of the pre-fit uncertainty in the total background and are centered around the pre-fit expectation value of the prediction. The hatched area shows the size of the post-fit uncertainty in the background prediction. The signal histogram template is shown as a solid line. The lower panel shows the relative difference of the observed number of events over the post-fit background prediction. 


\begin{tabular}{|lcccc|}
\hline Channel & Best fit $\mu$ & $\begin{array}{c}\text { Best fit } \sigma_{\mathrm{t} \overline{\mathrm{t}} \overline{\mathrm{t}}} \\
(\mathrm{fb})\end{array}$ & $\begin{array}{c}\text { Exp. significance } \\
\text { s.d. }\end{array}$ & $\begin{array}{c}\text { Obs. significance } \\
\text { s.d. }\end{array}$ \\
\hline Single-lepton & $1.6_{-1.6}^{+4.6}$ & $15_{-15}^{+42}$ & 0.21 & 0.36 \\
OS dilepton & $0.0^{+2.7}$ & $0^{+25}$ & 0.36 & 0.0 \\
Combined & $0.0^{+2.2}$ & $0^{+20}$ & 0.40 & 0.0 \\
(this analysis) & & & & 1.6 \\
SS dilepton + multilepton & $1.8_{-1.2}^{+1.5}$ & $17_{-11}^{+14}$ & 1.0 & 1.4 \\
Combined & $1.4_{-1.0}^{+1.2}$ & $13_{-9}^{+11}$ & 1.1 & \\
(this analysis $+[21])$ & & & & \\
\hline
\end{tabular}

Table 2. Maximum-likelihood signal strength, $\mu$, and cross section estimates, as well as the expected and observed significance of SM t $\bar{t} t \bar{t}$ production. Both $\mu$ and $\sigma_{\mathrm{t} \overline{\mathrm{t}} \mathrm{t} \overline{\mathrm{t}}}$ are constrained to be positive. The results for the two analyses from this paper are shown separately and combined. The results from a previous CMS multilepton measurement are also given [21]. The values quoted for the uncertainties on the signal strengths and cross sections are the one standard deviation (s.d.) values and include all statistical and systematic uncertainties. The expected significance is calculated assuming that the data are distributed according to the prediction of the SM with nominal t $\bar{t} t \bar{t}$ production cross section value $\sigma_{\mathrm{t} \overline{\mathrm{t}} \mathrm{t}}^{\mathrm{SM}}$, which corresponds to the assumed signal strength modifier value $\mu=1$.

\begin{tabular}{|lcccc|}
\hline Channel & Expected limit, $\mu$ & Observed limit, $\mu$ & $\begin{array}{c}\text { Expected limit } \\
(\mathrm{fb})\end{array}$ & $\begin{array}{c}\text { Observed limit } \\
(\mathrm{fb})\end{array}$ \\
\hline Single-lepton & $9.4_{-2.9}^{+4.4}$ & 10.6 & $86_{-26}^{+40}$ & 97 \\
OS dilepton & $7.3_{-2.5}^{+4.5}$ & 6.9 & $67_{-23}^{+41}$ & 64 \\
Combined & $5.7_{-1.8}^{+2.9}$ & 5.2 & $52_{-17}^{+26}$ & 48 \\
(this analysis) & & & & \\
SS dilepton + multilepton & $2.5_{-0.8}^{+1.4}$ & 4.6 & $21_{-7}^{+11}$ & 42 \\
Combined & $2.2_{-0.7}^{+1.1}$ & 3.6 & $20_{-6}^{+10}$ & 33 \\
(this analysis $+[21]$ ) & & & & \\
\hline
\end{tabular}

Table 3. Expected and observed 95\% CL upper limits on SM t $\overline{\mathrm{t}} \mathrm{t} \overline{\mathrm{t}}$ production as a multiple of $\sigma_{\mathrm{t} \overline{\mathrm{t}} \mathrm{t} \overline{\mathrm{t}}}^{\mathrm{SM}}$ and in $\mathrm{fb}$. The results for the two analyses from this paper are shown separately and combined. The results from a previous CMS multilepton search are also given [21]. The values quoted for the uncertainties in the expected limits indicate the regions containing $68 \%$ of the distribution of limits expected under the background-only hypothesis. The expected upper limits are calculated assuming that the data are distributed according to the prediction of the background-only model corresponding to the scenario with signal strength modifier value $\mu=0$.

\subsection{Combination with the same-sign dilepton and multileptons channels}

An independent search for the SM t $\bar{t} t \bar{t}$ production has been performed previously in samesign dilepton and multilepton channels [21]. This search is characterized by a different background composition which, in contrast to the single-lepton and opposite-sign dilepton

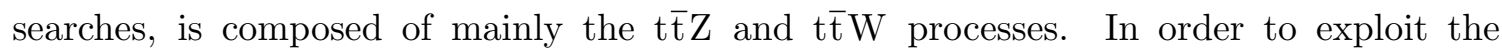
complementarity of this analysis, a combination of the results from single-lepton, oppositesign, same-sign and multilepton channels has been performed. The combination is based on 
a binned likelihood function equal to a product of likelihood terms over all search regions considered in single-lepton, opposite-sign and same-sign dilepton and multilepton channels.

Because of different origins of the dominant background processes, the main systematic uncertainties in the three analyses are independent and can be treated as uncorrelated. Nevertheless, the stability of the combination with respect to the assumption on the correlations between common sources of systematic uncertainty was tested by repeating the fit with and without correlations between the corresponding nuisance parameters. The resulting changes in the signal strength and expected limit were found to be less than $1 \%$ of the corresponding total uncertainties and were therefore not included.

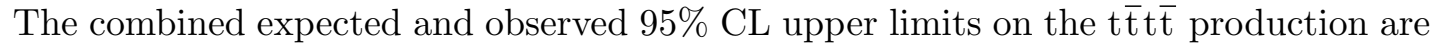
$20_{-6}^{+10} \mathrm{fb}$ and $33 \mathrm{fb}$, respectively, which is about a $10 \%$ improvement on the precision of the measurement with respect to the multilepton analysis alone. A summary of upper limit determinations from the individual analyses and their combination is provided in table 3 .

\subsection{Effective field theory interpretation}

New physics may manifest itself as modified interactions of SM fields, even if the associated particles are too heavy to be directly probed at the LHC. Such interactions can be modeled by extending the SM Lagrangian with terms involving composite operators of SM fields. Assuming that these terms preserve the gauge symmetries of the SM, possible new interactions can be classified according to their scaling dimension and the SM fields content [86-88]. The EFT Lagrangian reads

$$
\mathcal{L}_{\mathrm{EFT}}=\mathcal{L}_{\mathrm{SM}}^{(4)}+\frac{1}{\Lambda} \sum_{k} C_{k}^{(5)} \mathcal{O}_{k}^{(5)}+\frac{1}{\Lambda^{2}} \sum_{k} C_{k}^{(6)} \mathcal{O}_{k}^{(6)}+\ldots
$$

where $\mathcal{L}_{\mathrm{SM}}^{(4)}$ is the SM Lagrangian, while $\mathcal{O}_{k}^{(n)}$ and $C_{k}^{(n)}$ denote dimension- $n$ (dim- $n$ ) composite operators and their coupling parameters, respectively. Each term in the sum is suppressed by $\Lambda^{n-4}$, where $\Lambda$ is an energy scale that characterizes the new physics and $n$ is the scaling dimension of the corresponding operator. The energy scale, $\Lambda$, is the scale below which on-shell effects of BSM physics can be neglected and is typically related to the mass scale of the hypothetical BSM states. The EFT approach is generic and, in principle, experimental constraints obtained within the EFT framework can be recast into bounds on parameters of any ultraviolet-complete new physics model.

The production of four top quarks is a unique signature that provides information about models that predict enhanced interactions of the third generation quarks, such as four-fermion $t \bar{t} t \bar{t}$ coupling. The dim- 5 operators do not contribute to $t \bar{t} t \bar{t}$ production because they do not couple to top quarks [89]. A minimal basis of composite dim-6 operators contributing in eq. (6.1) was derived in ref. [87]. Only a small subset of these operators lead to four top quark production at LO in the EFT perturbation series. In a restricted scenario [10,90], assuming that new physics couples predominantly to the left-handed doublet and right-handed up-type quark singlet of the third generation, only four operators 


\begin{tabular}{|lc|cccc|}
\hline & $\sigma_{k}^{(1)}$ & & & $\sigma_{j, k}^{(2)}$ & \\
Operator & & $\mathcal{O}_{\mathrm{tt}}^{1}$ & $\mathcal{O}_{\mathrm{QQ}}^{1}$ & $\mathcal{O}_{\mathrm{Qt}}^{1}$ & $\mathcal{O}_{\mathrm{Qt}}^{8}$ \\
\hline $\mathcal{O}_{\mathrm{tt}}^{1}$ & 0.39 & 5.59 & 0.36 & -0.39 & 0.3 \\
$\mathcal{O}_{\mathrm{QQ}}^{1}$ & 0.47 & & 5.49 & -0.45 & 0.13 \\
$\mathcal{O}_{\mathrm{Qt}}^{1}$ & 0.03 & & & 1.9 & -0.08 \\
$\mathcal{O}_{\mathrm{Qt}}^{8}$ & 0.28 & & & & 0.45 \\
\hline
\end{tabular}

Table 4. Linear (left) and quadratic (right) parameterization coefficients, $\sigma_{k}^{(1)}$ and $\sigma_{j, k}^{(2)}$, of eq. (6.3). The coefficients $\sigma_{k}^{(1)}$ are in units $\left(\mathrm{fb} \mathrm{TeV}^{2}\right)$, while the coefficients $\sigma_{j, k}^{(2)}$ are in units $\left(\mathrm{fb} \mathrm{TeV}^{4}\right)$.

are expected to contribute significantly to $t \bar{t} t \bar{t}$ production, namely,

$$
\begin{aligned}
\mathcal{O}_{\mathrm{tt}}^{1} & =\left(\overline{\mathrm{t}}_{\mathrm{R}} \gamma^{\mu} \mathrm{t}_{\mathrm{R}}\right)\left(\overline{\mathrm{t}}_{\mathrm{R}} \gamma_{\mu} \mathrm{t}_{\mathrm{R}}\right), \\
\mathcal{O}_{\mathrm{QQ}}^{1} & =\left(\overline{\mathrm{Q}}_{\mathrm{L}} \gamma^{\mu} \mathrm{Q}_{\mathrm{L}}\right)\left(\overline{\mathrm{Q}}_{\mathrm{L}} \gamma_{\mu} \mathrm{Q}_{\mathrm{L}}\right), \\
\mathcal{O}_{\mathrm{Qt}}^{1} & =\left(\overline{\mathrm{Q}}_{\mathrm{L}} \gamma^{\mu} \mathrm{Q}_{\mathrm{L}}\right)\left(\overline{\mathrm{t}}_{\mathrm{R}} \gamma_{\mu} \mathrm{t}_{\mathrm{R}}\right), \\
\mathcal{O}_{\mathrm{Qt}}^{8} & =\left(\overline{\mathrm{Q}}_{\mathrm{L}} \gamma^{\mu} T^{\mathrm{A}} \mathrm{Q}_{\mathrm{L}}\right)\left(\overline{\mathrm{t}}_{\mathrm{R}} \gamma_{\mu} T^{\mathrm{A}} \mathrm{t}_{\mathrm{R}}\right),
\end{aligned}
$$

where $\mathrm{Q}_{\mathrm{L}}$ and $\mathrm{t}_{\mathrm{R}}$ denote the left-handed third generation quark doublet and the righthanded top quark singlet, respectively. The 4-fermion $t \bar{t} b \bar{b}$ operators were not included because of the negligible $\mathrm{b}$ quark parton density in the proton. Leading order predictions for the $\mathrm{pp} \rightarrow \mathrm{t} \overline{\mathrm{t}} \mathrm{t} \overline{\mathrm{t}}$ cross section can be parameterized using the equation

$$
\sigma_{\mathrm{t} \overline{\mathrm{t}} \overline{\mathrm{t}}}=\sigma_{\mathrm{t} \overline{\mathrm{t}} \overline{\mathrm{t}}}^{\mathrm{SM}}+\frac{1}{\Lambda^{2}} \sum_{k} C_{k} \sigma_{k}^{(1)}+\frac{1}{\Lambda^{4}} \sum_{j \leq k} C_{j} C_{k} \sigma_{j, k}^{(2)},
$$

where the linear terms, $C_{k} \sigma_{k}^{(1)}$, represent the interference of the SM production with the dim-6 EFT contribution, while the quadratic terms include two components: the square of the diagrams containing one EFT operator, and the interference term for two diagrams, each with one EFT operator. Representing $C_{k}$ as a column-vector, $\overrightarrow{\mathbf{C}}$, eq. (6.3) can be expressed in a matrix form as

$$
\sigma_{\mathrm{t} \overline{\mathrm{t}} \overline{\mathrm{t}}}=\sigma_{\mathrm{t} \overline{\mathrm{t}} \overline{\mathrm{t}}}^{\mathrm{SM}}+\frac{1}{\Lambda^{2}} \overrightarrow{\boldsymbol{C}}^{\boldsymbol{T}} \cdot \overrightarrow{\boldsymbol{\sigma}}^{(1)}+\frac{1}{\Lambda^{4}} \overrightarrow{\mathbf{C}}^{\mathbf{T}} \boldsymbol{\sigma}^{(2)} \overrightarrow{\boldsymbol{C}} .
$$

In order to find $\overrightarrow{\boldsymbol{\sigma}}^{(1)}$ and $\boldsymbol{\sigma}^{(2)}$, a system of linear equations has to be solved. It is obtained by substituting linearly-independent vectors $\vec{C}$ into eq. (6.4). In the cross section calculation, the EFT interactions are implemented in the FEYNRULES [90, 91] package and interfaced with MadGraph5_amC@NLO [13]. The NNPDF3.0LO [50] PDF set and $\alpha_{S}\left(M_{\mathrm{Z}}\right)=0.138$ were used in the calculation. In the EFT predictions, the SM contribution, $\sigma_{\mathrm{tt} t \bar{t}}^{\mathrm{SM}}$ in eqs. $(6.3)$ and (6.4), was rescaled to the NLO cross section of $9.2 \mathrm{fb}$ for the collision energy of $13 \mathrm{TeV}$. The linear and quadratic coefficients, $\sigma_{k}^{(1)}$ and $\sigma_{j, k}^{(2)}$, in eq. (6.3) can be found in table 4 .

The observed limit of $3.6 \sigma_{\mathrm{t} \overline{\mathrm{t} t} \overline{\mathrm{t}}}^{\mathrm{SM}}$, with a corresponding expected limit of $3.2 \sigma_{\mathrm{t} \overline{\mathrm{t}} \mathrm{t} \overline{\mathrm{t}}}^{\mathrm{SM}}$ (assuming $\mu=1$ ), from the combined experimental results, is used to constrain possible 


\begin{tabular}{|lcc|}
\hline Operator & Expected $C_{k} / \Lambda^{2}\left(\mathrm{TeV}^{-2}\right)$ & Observed $\left(\mathrm{TeV}^{-2}\right)$ \\
\hline $\mathcal{O}_{\mathrm{tt}}^{1}$ & {$[-2.0,1.8]$} & {$[-2.1,2.0]$} \\
$\mathcal{O}_{\mathrm{QQ}}^{1}$ & {$[-2.0,1.8]$} & {$[-2.2,2.0]$} \\
$\mathcal{O}_{\mathrm{Qt}}^{1}$ & {$[-3.3,3.2]$} & {$[-3.5,3.5]$} \\
$\mathcal{O}_{\mathrm{Qt}}^{8}$ & {$[-7.3,6.1]$} & {$[-7.9,6.6]$} \\
\hline
\end{tabular}

Table 5. Expected and observed 95\% CL intervals for selected coupling parameters. The intervals are extracted from upper limit on the $t \bar{t} t \bar{t}$ production cross section in the EFT model, where only one selected operator has a nonvanishing contribution.

\begin{tabular}{|lcc|}
\hline Operator & Expected $C_{k} / \Lambda^{2}\left(\mathrm{TeV}^{-2}\right)$ & Observed $\left(\mathrm{TeV}^{-2}\right)$ \\
\hline $\mathcal{O}_{\mathrm{tt}}^{1}$ & {$[-2.0,1.9]$} & {$[-2.2,2.1]$} \\
$\mathcal{O}_{\mathrm{QQ}}^{1}$ & {$[-2.0,1.9]$} & {$[-2.2,2.0]$} \\
$\mathcal{O}_{\mathrm{Qt}}^{1}$ & {$[-3.4,3.3]$} & {$[-3.7,3.5]$} \\
$\mathcal{O}_{\mathrm{Qt}}^{8}$ & {$[-7.4,6.3]$} & {$[-8.0,6.8]$} \\
\hline
\end{tabular}

Table 6. Expected and observed 95\% CL intervals for selected coupling parameters when contribution of other operators is marginalized.

contributions of EFT operators. Since the data are only sensitive to the ratios, $C_{k} / \Lambda^{2}$, the constraints are presented only for such ratios. In the limit setting, SM kinematics of the $t \bar{t} t \bar{t}$ final state were assumed and only rate information was utilized to calculate the constraints. Besides the NLO scale uncertainty from the SM t $\bar{t} t \bar{t}$ NLO prediction, no further scale uncertainties were added because other uncertainties on $t \bar{t} t \bar{t}$ production are already included in the experimental limit.

Independent limits were obtained under the assumption that only one operator contributes to the $t \bar{t} t \bar{t}$ cross section with the coefficients of the other operators set to zero. The intervals obtained are summarized in table 5. More conservative estimates were obtained by marginalizing the contribution of other operators within the interval $C_{k} / \Lambda^{2} \in[-4 \pi, 4 \pi]$, defined by the stability of perturbation series. The corresponding limits are listed in table 6 . The results obtained are only slightly weaker than independent constraints because of the small correlations between the operators.

As shown in tables 5 and 6 , the data have highest sensitivity to the contribution of $\mathcal{O}_{\mathrm{tt}}^{1}$ and $\mathcal{O}_{\mathrm{QQ}}^{1}$. The allowed intervals for the coupling parameters are almost independent of the other considered operators and stay stable after marginalization.

\section{Summary}

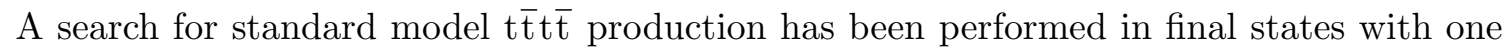
or two oppositely signed muons or electrons plus jets. The observed yields attributed to $t \bar{t} t \bar{t}$ production are consistent with the background predictions. An upper limit at $95 \%$ confidence level of $48 \mathrm{fb}$ is set on the cross section for $t \bar{t} t \bar{t}$ production. Combining this result with a previous same-sign dilepton and multilepton search [21] the resulting cross 
section is $13_{-9}^{+11} \mathrm{fb}$ with an observed significance of 1.4 standard deviations. The combined result constitutes one of the most stringent constraints from CMS on the production of four top quarks and can be used for phenomenological reinterpretation of a wide range of new physics models. The experimental results are interpreted in the effective field theory framework and yield limits on dimension- 6 four-fermion operators coupling to third generation quarks competitive with the latest ATLAS interpretation [18].

\section{Acknowledgments}

We congratulate our colleagues in the CERN accelerator departments for the excellent performance of the LHC and thank the technical and administrative staffs at CERN and at other CMS institutes for their contributions to the success of the CMS effort. In addition, we gratefully acknowledge the computing centers and personnel of the Worldwide LHC Computing Grid for delivering so effectively the computing infrastructure essential to our analyses. Finally, we acknowledge the enduring support for the construction and operation of the LHC and the CMS detector provided by the following funding agencies: BMBWF and FWF (Austria); FNRS and FWO (Belgium); CNPq, CAPES, FAPERJ, FAPERGS, and FAPESP (Brazil); MES (Bulgaria); CERN; CAS, MoST, and NSFC (China); COLCIENCIAS (Colombia); MSES and CSF (Croatia); RPF (Cyprus); SENESCYT (Ecuador); MoER, ERC IUT, PUT and ERDF (Estonia); Academy of Finland, MEC, and HIP (Finland); CEA and CNRS/IN2P3 (France); BMBF, DFG, and HGF (Germany); GSRT (Greece); NKFIA (Hungary); DAE and DST (India); IPM (Iran); SFI (Ireland); INFN (Italy); MSIP and NRF (Republic of Korea); MES (Latvia); LAS (Lithuania); MOE and UM (Malaysia); BUAP, CINVESTAV, CONACYT, LNS, SEP, and UASLP-FAI (Mexico); MOS (Montenegro); MBIE (New Zealand); PAEC (Pakistan); MSHE and NSC (Poland); FCT (Portugal); JINR (Dubna); MON, RosAtom, RAS, RFBR, and NRC KI (Russia); MESTD (Serbia); SEIDI, CPAN, PCTI, and FEDER (Spain); MOSTR (Sri Lanka); Swiss Funding Agencies (Switzerland); MST (Taipei); ThEPCenter, IPST, STAR, and NSTDA (Thailand); TUBITAK and TAEK (Turkey); NASU and SFFR (Ukraine); STFC (United Kingdom); DOE and NSF (U.S.A.).

Individuals have received support from the Marie-Curie program and the European Research Council and Horizon 2020 Grant, contract Nos. 675440, 752730, and 765710 (European Union); the Leventis Foundation; the A.P. Sloan Foundation; the Alexander von Humboldt Foundation; the Belgian Federal Science Policy Office; the Fonds pour la Formation à la Recherche dans l'Industrie et dans l'Agriculture (FRIA-Belgium); the Agentschap voor Innovatie door Wetenschap en Technologie (IWT-Belgium); the F.R.S.-FNRS and FWO (Belgium) under the "Excellence of Science - EOS" — be.h project n. 30820817; the Beijing Municipal Science \& Technology Commission, No. Z181100004218003; the Ministry of Education, Youth and Sports (MEYS) of the Czech Republic; the Lendület ("Momentum") Program and the János Bolyai Research Scholarship of the Hungarian Academy of Sciences, the New National Excellence Program ÚNKP, the NKFIA research grants 123842, 123959, 124845, 124850, 125105, 128713, 128786, and 129058 (Hungary); the Council of Science and Industrial Research, India; the HOMING PLUS program of 
the Foundation for Polish Science, cofinanced from European Union, Regional Development Fund, the Mobility Plus program of the Ministry of Science and Higher Education, the National Science Center (Poland), contracts Harmonia 2014/14/M/ST2/00428, Opus 2014/13/B/ST2/02543, 2014/15/B/ST2/03998, and 2015/19/B/ST2/02861, Sonatabis 2012/07/E/ST2/01406; the National Priorities Research Program by Qatar National Research Fund; the Ministry of Science and Education, grant no. 3.2989.2017 (Russia); the Programa Estatal de Fomento de la Investigación Científica y Técnica de Excelencia María de Maeztu, grant MDM-2015-0509 and the Programa Severo Ochoa del Principado de Asturias; the Thalis and Aristeia programs cofinanced by EU-ESF and the Greek NSRF; the Rachadapisek Sompot Fund for Postdoctoral Fellowship, Chulalongkorn University and the Chulalongkorn Academic into Its 2nd Century Project Advancement Project (Thailand); the Welch Foundation, contract C-1845; and the Weston Havens Foundation (U.S.A.).

Open Access. This article is distributed under the terms of the Creative Commons Attribution License (CC-BY 4.0), which permits any use, distribution and reproduction in any medium, provided the original author(s) and source are credited.

\section{References}

[1] H.P. Nilles, Supersymmetry, supergravity and particle physics, Phys. Rept. 110 (1984) 1 [INSPIRE].

[2] S.P. Martin, A supersymmetry primer, in Perspectives on supersymmetry II, G.L. Kane, ed., Adv. Ser. Direct. High Energy Phys. volume 21, World Scientific, Singapore (2010).

[3] G. Cacciapaglia et al., Composite scalars at the LHC: the Higgs, the sextet and the octet, JHEP 11 (2015) 201 [arXiv: 1507. 02283] [INSPIRE].

[4] K. Kumar, T.M.P. Tait and R. Vega-Morales, Manifestations of top compositeness at colliders, JHEP 05 (2009) 022 [arXiv:0901.3808] [INSPIRE].

[5] D. Dicus, A. Stange and S. Willenbrock, Higgs decay to top quarks at hadron colliders, Phys. Lett. B 333 (1994) 126 [hep-ph/9404359] [INSPIRE].

[6] N. Craig, F. D'Eramo, P. Draper, S. Thomas and H. Zhang, The hunt for the rest of the Higgs bosons, JHEP 06 (2015) 137 [arXiv: 1504.04630] [INSPIRE].

[7] N. Craig, J. Hajer, Y.-Y. Li, T. Liu and H. Zhang, Heavy Higgs bosons at low $\tan \beta$ : from the LHC to $100 \mathrm{TeV}$, JHEP 01 (2017) 018 [arXiv:1605.08744] [INSPIRE].

[8] G. Cacciapaglia, A. Deandrea and J. Llodra-Perez, A dark matter candidate from Lorentz invariance in 6D, JHEP 03 (2010) 083 [arXiv:0907.4993] [INSPIRE].

[9] O. Ducu, L. Heurtier and J. Maurer, LHC signatures of a $Z^{\prime}$ mediator between dark matter and the $\mathrm{SU}(3)$ sector, JHEP 03 (2016) 006 [arXiv: 1509.05615] [INSPIRE].

[10] C. Degrande et al., Non-resonant new physics in top pair production at hadron colliders, JHEP 03 (2011) 125 [arXiv: 1010.6304] [INSPIRE].

[11] R. Frederix, D. Pagani and M. Zaro, Large NLO corrections in $t \bar{t} W^{ \pm}$and $t \bar{t} t \bar{t}$ hadroproduction from supposedly subleading EW contributions, JHEP 02 (2018) 031 [arXiv: 1711.02116] [INSPIRE]. 
[12] G. Bevilacqua and M. Worek, Constraining BSM physics at the LHC: four top final states with NLO accuracy in perturbative QCD, JHEP 07 (2012) 111 [arXiv:1206.3064] [INSPIRE].

[13] J. Alwall, et al., The automated computation of tree-level and next-to-leading order differential cross sections and their matching to parton shower simulations, JHEP 07 (2014) 079 [arXiv: 1405.0301] [inSPIRE].

[14] ATLAS collaboration, Search for supersymmetry at $\sqrt{s}=8 \mathrm{TeV}$ in final states with jets and two same-sign leptons or three leptons with the ATLAS detector, JHEP 06 (2014) 035 [arXiv: 1404.2500] [INSPIRE].

[15] ATLAS collaboration, Search for production of vector-like quark pairs and of four top quarks in the lepton-plus-jets final state in pp collisions at $\sqrt{s}=8 \mathrm{TeV}$ with the ATLAS detector, JHEP 08 (2015) 105 [arXiv: 1505. 04306] [INSPIRE].

[16] ATLAS collaboration, Analysis of events with b-jets and a pair of leptons of the same charge in pp collisions at $\sqrt{s}=8 \mathrm{TeV}$ with the ATLAS detector, JHEP 10 (2015) 150 [arXiv: 1504.04605] [INSPIRE].

[17] CMS collaboration, Search for standard model production of four top quarks in the lepton + jets channel in pp collisions at $\sqrt{s}=8 \mathrm{TeV}$, JHEP 11 (2014) 154 [arXiv:1409.7339] [INSPIRE].

[18] ATLAS collaboration, Search for four-top-quark production in the single-lepton and opposite-sign dilepton final states in pp collisions at $\sqrt{s}=13$ TeV with the ATLAS detector, Phys. Rev. D 99 (2019) 052009 [arXiv: 1811.02305] [INSPIRE].

[19] ATLAS collaboration, Search for new phenomena in events with same-charge leptons and $b$-jets in pp collisions at $\sqrt{s}=13 \mathrm{TeV}$ with the ATLAS detector, JHEP 12 (2018) 039 [arXiv: 1807.11883] [INSPIRE].

[20] CMS collaboration, Search for standard model production of four top quarks in proton-proton collisions at $\sqrt{s}=13 \mathrm{TeV}$, Phys. Lett. B 772 (2017) 336 [arXiv:1702.06164] [INSPIRE].

[21] CMS collaboration, Search for standard model production of four top quarks with same-sign and multilepton final states in proton-proton collisions at $\sqrt{s}=13$ TeV, Eur. Phys. J. C 78 (2018) 140 [arXiv:1710.10614] [INSPIRE].

[22] CMS collaboration, Search for physics beyond the standard model in events with two leptons of same sign, missing transverse momentum and jets in proton-proton collisions at $\sqrt{s}=13 \mathrm{TeV}$, Eur. Phys. J. C 77 (2017) 578 [arXiv:1704.07323] [INSPIRE].

[23] ATLAS collaboration, Search for pair production of up-type vector-like quarks and for four-top-quark events in final states with multiple b-jets with the ATLAS detector, JHEP 07 (2018) 089 [arXiv: 1803.09678] [INSPIRE].

[24] CMS collaboration, The CMS experiment at the CERN LHC, 2008 JINST 3 S08004 [INSPIRE].

[25] M.L. Mangano, M. Moretti, F. Piccinini and M. Treccani, Matching matrix elements and shower evolution for top-quark production in hadronic collisions, JHEP 01 (2007) 013 [hep-ph/0611129] [INSPIRE].

[26] P. Artoisenet, R. Frederix, O. Mattelaer and R. Rietkerk, Automatic spin-entangled decays of heavy resonances in Monte Carlo simulations, JHEP 03 (2013) 015 [arXiv:1212.3460] [INSPIRE]. 
[27] S. Frixione, P. Nason and G. Ridolfi, A positive-weight next-to-leading-order Monte Carlo for heavy flavour hadroproduction, JHEP 09 (2007) 126 [arXiv:0707.3088] [INSPIRE].

[28] P. Nason, A new method for combining NLO QCD with shower Monte Carlo algorithms, JHEP 11 (2004) 040 [hep-ph/0409146] [INSPIRE].

[29] S. Frixione, P. Nason and C. Oleari, Matching NLO QCD computations with Parton Shower simulations: the POWHEG method, JHEP 11 (2007) 070 [arXiv: 0709.2092] [INSPIRE].

[30] S. Alioli, P. Nason, C. Oleari and E. Re, A general framework for implementing NLO calculations in shower Monte Carlo programs: the POWHEG BOX, JHEP 06 (2010) 043 [arXiv: 1002.2581] [INSPIRE].

[31] S. Alioli, S.-O. Moch and P. Uwer, Hadronic top-quark pair-production with one jet and parton showering, JHEP 01 (2012) 137 [arXiv:1110.5251] [INSPIRE].

[32] M. Czakon and A. Mitov, Top++: a program for the calculation of the top-pair cross-section at hadron colliders, Comput. Phys. Commun. 185 (2014) 2930 [arXiv:1112.5675] [InSPIRE].

[33] M. Beneke, P. Falgari, S. Klein and C. Schwinn, Hadronic top-quark pair production with NNLL threshold resummation, Nucl. Phys. B 855 (2012) 695 [arXiv:1109.1536] [INSPIRE].

[34] M. Cacciari et al., Top-pair production at hadron colliders with next-to-next-to-leading logarithmic soft-gluon resummation, Phys. Lett. B 710 (2012) 612 [arXiv:1111.5869] [INSPIRE].

[35] P. Bärnreuther, M. Czakon and A. Mitov, Percent level precision physics at the Tevatron: first genuine NNLO QCD corrections to $q \bar{q} \rightarrow t \bar{t}+X$, Phys. Rev. Lett. 109 (2012) 132001 [arXiv: 1204.5201] [INSPIRE].

[36] M. Czakon and A. Mitov, NNLO corrections to top-pair production at hadron colliders: the all-fermionic scattering channels, JHEP 12 (2012) 054 [arXiv:1207.0236] [INSPIRE].

[37] M. Czakon and A. Mitov, NNLO corrections to top pair production at hadron colliders: the quark-gluon reaction, JHEP 01 (2013) 080 [arXiv:1210.6832] [INSPIRE].

[38] M. Czakon, P. Fiedler and A. Mitov, Total top-quark pair-production cross section at hadron colliders through $O\left(\alpha_{S}^{4}\right)$, Phys. Rev. Lett. 110 (2013) 252004 [arXiv:1303.6254] [INSPIRE].

[39] CMS collaboration, Investigations of the impact of the parton shower tuning in PYTHIA 8 in the modelling of $t \bar{t}$ at $\sqrt{s}=8$ and $13 \mathrm{TeV}$, CMS-PAS-TOP-16-021 (2016).

[40] CMS collaboration, Event generator tunes obtained from underlying event and multiparton scattering measurements, Eur. Phys. J. C 76 (2016) 155 [arXiv:1512.00815] [inSPIRE].

[41] P. Skands, S. Carrazza and J. Rojo, Tuning PYTHIA 8.1: the Monash 2013 Tune, Eur. Phys. J. C 74 (2014) 3024 [arXiv:1404.5630] [INSPIRE].

[42] CMS collaboration, Measurements of $t \bar{t}$ differential cross sections in proton-proton collisions at $\sqrt{s}=13 \mathrm{TeV}$ using events containing two leptons, JHEP 02 (2019) 149 [arXiv: 1811.06625] [INSPIRE].

[43] CMS collaboration, Measurement of differential cross sections for the production of top quark pairs and of additional jets in lepton+jets events from pp collisions at $\sqrt{s}=13 \mathrm{TeV}$, Phys. Rev. D 97 (2018) 112003 [arXiv:1803.08856] [INSPIRE].

[44] E. Re, Single-top Wt-channel production matched with parton showers using the POWHEG method, Eur. Phys. J. C 71 (2011) 1547 [arXiv:1009.2450] [inSPIRE]. 
[45] M. Aliev et al., HATHOR: HAdronic Top and Heavy quarks crOss section calculatoR, Comput. Phys. Commun. 182 (2011) 1034 [arXiv:1007.1327] [InSPIRE].

[46] P. Kant et al., HatHor for single top-quark production: updated predictions and uncertainty estimates for single top-quark production in hadronic collisions, Comput. Phys. Commun. 191 (2015) 74 [arXiv: 1406.4403] [INSPIRE].

[47] J. Alwall et al., Comparative study of various algorithms for the merging of parton showers and matrix elements in hadronic collisions, Eur. Phys. J. C 53 (2008) 473 [arXiv:0706.2569] [INSPIRE].

[48] Y. Li and F. Petriello, Combining QCD and electroweak corrections to dilepton production in FEWZ, Phys. Rev. D 86 (2012) 094034 [arXiv:1208.5967] [INSPIRE].

[49] LHC Higgs Cross Section Working Group collaboration, Handbook of LHC Higgs Cross Sections: 4. Deciphering the nature of the Higgs sector, arXiv:1610.07922 [INSPIRE].

[50] NNPDF collaboration, Parton distributions for the LHC Run II, JHEP 04 (2015) 040 [arXiv: 1410.8849] [INSPIRE].

[51] T. Sjöstrand et al., An introduction to PYTHIA 8.2, Comput. Phys. Commun. 191 (2015) 159 [arXiv: 1410.3012] [INSPIRE].

[52] CMS collaboration, Measurement of jet substructure observables in t $\bar{t}$ events from proton-proton collisions at $\sqrt{s}=13$ TeV, Phys. Rev. D 98 (2018) 092014 [arXiv: 1808.07340] [INSPIRE].

[53] CMS collaboration, Measurements of differential cross sections of top quark pair production as a function of kinematic event variables in proton-proton collisions at $\sqrt{s}=13 \mathrm{TeV}$, JHEP 06 (2018) 002 [arXiv: 1803.03991] [INSPIRE].

[54] CMS collaboration, Investigations of the impact of the parton shower tuning in PYTHIA 8 in the modelling of $t \bar{t}$ at $\sqrt{s}=8$ and 13 TeV, CMS-PAS-TOP-16-021 (2016).

[55] GEANT4 collaboration, GEANT4 - a simulation toolkit, Nucl. Instrum. Meth. A 506 (2003) 250 [INSPIRE].

[56] CMS collaboration, The CMS trigger system, 2017 JINST 12 P01020 [arXiv:1609. 02366] [INSPIRE].

[57] CMS collaboration, Particle-flow reconstruction and global event description with the CMS detector, 2017 JINST 12 P10003 [arXiv:1706. 04965] [INSPIRE].

[58] CMS collaboration, Performance of the CMS muon detector and muon reconstruction with proton-proton collisions at $\sqrt{s}=13 \mathrm{TeV}, 2018$ JINST 13 P06015 [arXiv: 1804.04528] [INSPIRE].

[59] CMS collaboration, Performance of electron reconstruction and selection with the CMS detector in proton-proton collisions at $\sqrt{s}=8 \mathrm{TeV}, 2015$ JINST $10 \mathrm{P} 06005$ [arXiv: 1502.02701] [INSPIRE].

[60] M. Cacciari, G.P. Salam and G. Soyez, The anti- $k_{t}$ jet clustering algorithm, JHEP 04 (2008) 063 [arXiv: 0802.1189] [INSPIRE].

[61] M. Cacciari, G.P. Salam and G. Soyez, FastJet user manual, Eur. Phys. J. C 72 (2012) 1896 [arXiv: 1111.6097] [INSPIRE].

[62] M. Cacciari, G.P. Salam and G. Soyez, The catchment area of jets, JHEP 04 (2008) 005 [arXiv:0802.1188] [INSPIRE]. 
[63] CMS collaboration, Pileup removal algorithms, CMS-PAS-JME-14-001 (2014).

[64] CMS collaboration, Jet energy scale and resolution in the CMS experiment in pp collisions at $8 \mathrm{TeV}, 2017$ JINST $12 \mathrm{P} 02014$ [arXiv: 1607.03663] [INSPIRE].

[65] CMS collaboration, Performance of missing transverse momentum in pp collisions at $\sqrt{s}=13$ TeV using the CMS detector, CMS-PAS-JME-17-001 (2017).

[66] CMS collaboration, Identification of heavy-flavour jets with the CMS detector in pp collisions at $13 \mathrm{TeV}, 2018$ JINST $13 \mathrm{P} 05011$ [arXiv:1712.07158] [INSPIRE].

[67] CMS collaboration, Search for $t \bar{t} H$ production in the $H \rightarrow b \bar{b}$ decay channel with leptonic $t \bar{t}$ decays in proton-proton collisions at $\sqrt{s}=13 \mathrm{TeV}$, JHEP 03 (2019) 026 [arXiv: 1804.03682] [INSPIRE].

[68] L. Breiman, J. Friedman, R.A. Olshen and C.J. Stone, Classification and regression trees, Chapman and Hall/CRC, U.S.A. (1984).

[69] J.H. Friedman, Recent advances in predictive (machine) learning, J. Classif. 23 (2006) 175.

[70] Y. Freund and R.E. Schapire, A decision-theoretic generalization of on-line learning and an application to boosting, J. Comput. Syst. Sci. 55 (1997) 119.

[71] A. Höcker et al., TMVA - Toolkit for Multivariate Data Analysis, PoS(ACAT) 040 [physics/0703039] [INSPIRE].

[72] J.D. Bjorken and S.J. Brodsky, Statistical Model for electron-Positron Annihilation Into Hadrons, Phys. Rev. D 1 (1970) 1416 [InSPIRE].

[73] CMS Collaboration, CMS luminosity measurements for the 2016 data taking period, CMS-PAS-LUM-17-001 (2017).

[74] CMS collaboration, Measurement of the inelastic proton-proton cross section at $\sqrt{s}=13$ TeV, JHEP 07 (2018) 161 [arXiv: 1802.02613] [INSPIRE].

[75] J. Butterworth et al., PDF 4 LHC recommendations for LHC Run II, J. Phys. G 43 (2016) 023001 [arXiv: 1510.03865] [INSPIRE].

[76] L.A. Harland-Lang, A.D. Martin, P. Motylinski and R.S. Thorne, Parton distributions in the LHC era: MMHT 2014 PDFs, Eur. Phys. J. C 75 (2015) 204 [arXiv: 1412.3989] [InSPIRE].

[77] S. Dulat et al., New parton distribution functions from a global analysis of quantum chromodynamics, Phys. Rev. D 93 (2016) 033006 [arXiv:1506.07443] [INSPIRE].

[78] CMS collaboration, Measurement of differential cross sections for top quark pair production using the lepton+jets final state in proton-proton collisions at $13 \mathrm{TeV}$, Phys. Rev. D 95 (2017) 092001 [arXiv: 1610.04191] [INSPIRE].

[79] CMS collaboration, Measurement of normalized differential $t \bar{t}$ cross sections in the dilepton channel from pp collisions at $\sqrt{s}=13 \mathrm{TeV}$, JHEP 04 (2018) 060 [arXiv:1708.07638] [INSPIRE].

[80] CMS collaboration, Measurements of $t \bar{t}$ cross sections in association with $b$ jets and inclusive jets and their ratio using dilepton final states in pp collisions at $\sqrt{s}=13 \mathrm{TeV}$, Phys. Lett. B 776 (2018) 355 [arXiv: 1705.10141] [INSPIRE].

[81] T. Junk, Confidence level computation for combining searches with small statistics, Nucl. Instrum. Meth. A 434 (1999) 435 [hep-ex/9902006] [INSPIRE]. 
[82] A.L. Read, Presentation of search results: the $C L_{s}$ technique, J. Phys. G 28 (2002) 2693 [INSPIRE].

[83] L. Moneta et al., The RooStats project, PoS (ACAT2010) 057 [arXiv:1009.1003] [INSPIRE].

[84] G. Cowan, K. Cranmer, E. Gross and O. Vitells, Asymptotic formulae for likelihood-based tests of new physics, Eur. Phys. J. C 71 (2011) 1554 [Erratum ibid. C 73 (2013) 2501] [arXiv: 1007.1727] [INSPIRE].

[85] ATLAS and CMS collaborations and the LHC Higgs Combination Group, Procedure for the LHC Higgs boson search combination in Summer 2011, CMS-NOTE-2011-005 (2011).

[86] W. Buchmüller and D. Wyler, Effective lagrangian analysis of new interactions and flavor conservation, Nucl. Phys. B 268 (1986) 621 [INSPIRE].

[87] B. Grzadkowski, M. Iskrzyński, M. Misiak and J. Rosiek, Dimension-six terms in the standard model Lagrangian, JHEP 10 (2010) 085 [arXiv: 1008.4884] [INSPIRE].

[88] N.P. Hartlannd et al., A Monte Carlo global analysis of the standard model effective field theory: the top quark sector, JHEP 04 (2019) 100 [arXiv: 1901.05965] [INSPIRE].

[89] S. Weinberg, Baryon and lepton nonconserving processes, Phys. Rev. Lett. 43 (1979) 1566 [INSPIRE].

[90] D. Barducci et al., Interpreting top-quark LHC measurements in the standard-model effective field theory, arXiv:1802.07237 [INSPIRE].

[91] A. Alloul et al., FeynRules 2.0 - A complete toolbox for tree-level phenomenology, Comput. Phys. Commun. 185 (2014) 2250 [arXiv:1310.1921] [INSPIRE]. 


\section{The CMS collaboration}

\section{Yerevan Physics Institute, Yerevan, Armenia}

A.M. Sirunyan ${ }^{\dagger}$, A. Tumasyan

\section{Institut für Hochenergiephysik, Wien, Austria}

W. Adam, F. Ambrogi, T. Bergauer, J. Brandstetter, M. Dragicevic, J. Erö, A. Escalante Del Valle, M. Flechl, R. Frühwirth ${ }^{1}$, M. Jeitler ${ }^{1}$, N. Krammer, I. Krätschmer, D. Liko, T. Madlener, I. Mikulec, N. Rad, J. Schieck ${ }^{1}$, R. Schöfbeck, M. Spanring, D. Spitzbart, W. Waltenberger, C.-E. Wulz ${ }^{1}$, M. Zarucki

Institute for Nuclear Problems, Minsk, Belarus

V. Drugakov, V. Mossolov, J. Suarez Gonzalez

\section{Universiteit Antwerpen, Antwerpen, Belgium}

M.R. Darwish, E.A. De Wolf, D. Di Croce, X. Janssen, J. Lauwers, A. Lelek, M. Pieters, H. Rejeb Sfar, H. Van Haevermaet, P. Van Mechelen, S. Van Putte, N. Van Remortel

\section{Vrije Universiteit Brussel, Brussel, Belgium}

F. Blekman, E.S. Bols, S.S. Chhibra, J. D'Hondt, J. De Clercq, D. Lontkovskyi, S. Lowette, I. Marchesini, S. Moortgat, L. Moreels, Q. Python, K. Skovpen, S. Tavernier, W. Van Doninck, P. Van Mulders, I. Van Parijs

\section{Université Libre de Bruxelles, Bruxelles, Belgium}

D. Beghin, B. Bilin, H. Brun, B. Clerbaux, G. De Lentdecker, H. Delannoy, B. Dorney,

L. Favart, A. Grebenyuk, A.K. Kalsi, J. Luetic, A. Popov, N. Postiau, E. Starling, L. Thomas, C. Vander Velde, P. Vanlaer, D. Vannerom, Q. Wang

\section{Ghent University, Ghent, Belgium}

T. Cornelis, D. Dobur, I. Khvastunov², C. Roskas, D. Trocino, M. Tytgat, W. Verbeke, B. Vermassen, M. Vit, N. Zaganidis

Université Catholique de Louvain, Louvain-la-Neuve, Belgium

O. Bondu, G. Bruno, C. Caputo, P. David, C. Delaere, M. Delcourt, A. Giammanco, V. Lemaitre, A. Magitteri, J. Prisciandaro, A. Saggio, M. Vidal Marono, P. Vischia, J. Zobec

\section{Centro Brasileiro de Pesquisas Fisicas, Rio de Janeiro, Brazil}

F.L. Alves, G.A. Alves, G. Correia Silva, C. Hensel, A. Moraes, P. Rebello Teles

Universidade do Estado do Rio de Janeiro, Rio de Janeiro, Brazil

E. Belchior Batista Das Chagas, W. Carvalho, J. Chinellato ${ }^{3}$, E. Coelho, E.M. Da Costa, G.G. Da Silveira ${ }^{4}$, D. De Jesus Damiao, C. De Oliveira Martins, S. Fonseca De Souza, L.M. Huertas Guativa, H. Malbouisson, J. Martins ${ }^{5}$, D. Matos Figueiredo, M. Medina Jaime ${ }^{6}$, M. Melo De Almeida, C. Mora Herrera, L. Mundim, H. Nogima, W.L. Prado Da Silva, L.J. Sanchez Rosas, A. Santoro, A. Sznajder, M. Thiel, E.J. Tonelli Manganote ${ }^{3}$, F. Torres Da Silva De Araujo, A. Vilela Pereira 
Universidade Estadual Paulista ${ }^{a}$, Universidade Federal do $\mathrm{ABC}^{b}$, São Paulo, Brazil

S. Ahuja ${ }^{a}$, C.A. Bernardes ${ }^{a}$, L. Calligaris ${ }^{a}$, T.R. Fernandez Perez Tomei ${ }^{a}$, E.M. Gregores ${ }^{b}$, D.S. Lemos, P.G. Mercadante ${ }^{b}$, S.F. Novaes ${ }^{a}$, SandraS. Padula ${ }^{a}$

Institute for Nuclear Research and Nuclear Energy, Bulgarian Academy of Sciences, Sofia, Bulgaria

A. Aleksandrov, G. Antchev, R. Hadjiiska, P. Iaydjiev, A. Marinov, M. Misheva, M. Rodozov, M. Shopova, G. Sultanov

University of Sofia, Sofia, Bulgaria

M. Bonchev, A. Dimitrov, T. Ivanov, L. Litov, B. Pavlov, P. Petkov

Beihang University, Beijing, China

W. Fang $^{7}$, X. $\mathrm{Gao}^{7}$, L. Yuan

Institute of High Energy Physics, Beijing, China

M. Ahmad, G.M. Chen, H.S. Chen, M. Chen, C.H. Jiang, D. Leggat, H. Liao, Z. Liu, S.M. Shaheen ${ }^{8}$, A. Spiezia, J. Tao, E. Yazgan, H. Zhang, S. Zhang ${ }^{8}$, J. Zhao

State Key Laboratory of Nuclear Physics and Technology, Peking University, Beijing, China

A. Agapitos, Y. Ban, G. Chen, A. Levin, J. Li, L. Li, Q. Li, Y. Mao, S.J. Qian, D. Wang Tsinghua University, Beijing, China

Z. Hu, Y. Wang

Universidad de Los Andes, Bogota, Colombia

C. Avila, A. Cabrera, L.F. Chaparro Sierra, C. Florez, C.F. González Hernández, M.A. Segura Delgado

Universidad de Antioquia, Medellin, Colombia

J. Mejia Guisao, J.D. Ruiz Alvarez, C.A. Salazar González, N. Vanegas Arbelaez

University of Split, Faculty of Electrical Engineering, Mechanical Engineering and Naval Architecture, Split, Croatia

D. Giljanović, N. Godinovic, D. Lelas, I. Puljak, T. Sculac

University of Split, Faculty of Science, Split, Croatia

Z. Antunovic, M. Kovac

Institute Rudjer Boskovic, Zagreb, Croatia

V. Brigljevic, S. Ceci, D. Ferencek, K. Kadija, B. Mesic, M. Roguljic, A. Starodumov ${ }^{9}$, T. Susa

University of Cyprus, Nicosia, Cyprus

M.W. Ather, A. Attikis, E. Erodotou, A. Ioannou, M. Kolosova, S. Konstantinou, G. Mavromanolakis, J. Mousa, C. Nicolaou, F. Ptochos, P.A. Razis, H. Rykaczewski, D. Tsiakkouri 
Charles University, Prague, Czech Republic

M. Finger ${ }^{10}$, M. Finger Jr. ${ }^{10}$, A. Kveton, J. Tomsa

Escuela Politecnica Nacional, Quito, Ecuador

E. Ayala

Universidad San Francisco de Quito, Quito, Ecuador

E. Carrera Jarrin

Academy of Scientific Research and Technology of the Arab Republic of Egypt, Egyptian Network of High Energy Physics, Cairo, Egypt

Y. Assran ${ }^{11,12}$, S. Elgammal ${ }^{12}$

National Institute of Chemical Physics and Biophysics, Tallinn, Estonia

S. Bhowmik, A. Carvalho Antunes De Oliveira, R.K. Dewanjee, K. Ehataht, M. Kadastik, M. Raidal, C. Veelken

Department of Physics, University of Helsinki, Helsinki, Finland

P. Eerola, L. Forthomme, H. Kirschenmann, K. Osterberg, M. Voutilainen

Helsinki Institute of Physics, Helsinki, Finland

F. Garcia, J. Havukainen, J.K. Heikkilä, T. Järvinen, V. Karimäki, R. Kinnunen,

T. Lampén, K. Lassila-Perini, S. Laurila, S. Lehti, T. Lindén, P. Luukka, T. Mäenpää,

H. Siikonen, E. Tuominen, J. Tuominiemi

Lappeenranta University of Technology, Lappeenranta, Finland

T. Tuuva

IRFU, CEA, Université Paris-Saclay, Gif-sur-Yvette, France

M. Besancon, F. Couderc, M. Dejardin, D. Denegri, B. Fabbro, J.L. Faure, F. Ferri, S. Ganjour, A. Givernaud, P. Gras, G. Hamel de Monchenault, P. Jarry, C. Leloup, E. Locci, J. Malcles, J. Rander, A. Rosowsky, M.Ö. Sahin, A. Savoy-Navarro ${ }^{13}$, M. Titov

Laboratoire Leprince-Ringuet, Ecole polytechnique, CNRS/IN2P3, Université Paris-Saclay, Palaiseau, France

C. Amendola, F. Beaudette, P. Busson, C. Charlot, B. Diab, G. Falmagne, R. Granier de Cassagnac, I. Kucher, A. Lobanov, C. Martin Perez, M. Nguyen, C. Ochando, P. Paganini, J. Rembser, R. Salerno, J.B. Sauvan, Y. Sirois, A. Zabi, A. Zghiche

Université de Strasbourg, CNRS, IPHC UMR 7178, Strasbourg, France

J.-L. Agram ${ }^{14}$, J. Andrea, D. Bloch, G. Bourgatte, J.-M. Brom, E.C. Chabert, C. Collard, E. Conte ${ }^{14}$, J.-C. Fontaine ${ }^{14}$, D. Gelé, U. Goerlach, M. Jansová, A.-C. Le Bihan, N. Tonon, P. Van Hove

Centre de Calcul de l'Institut National de Physique Nucleaire et de Physique des Particules, CNRS/IN2P3, Villeurbanne, France

S. Gadrat 
Université de Lyon, Université Claude Bernard Lyon 1, CNRS-IN2P3, Institut de Physique Nucléaire de Lyon, Villeurbanne, France

S. Beauceron, C. Bernet, G. Boudoul, C. Camen, N. Chanon, R. Chierici, D. Contardo, P. Depasse, H. El Mamouni, J. Fay, S. Gascon, M. Gouzevitch, B. Ille, Sa. Jain, F. Lagarde, I.B. Laktineh, H. Lattaud, M. Lethuillier, L. Mirabito, S. Perries, V. Sordini, G. Touquet, M. Vander Donckt, S. Viret

Georgian Technical University, Tbilisi, Georgia

A. Khvedelidze ${ }^{10}$

Tbilisi State University, Tbilisi, Georgia

Z. Tsamalaidze ${ }^{10}$

RWTH Aachen University, I. Physikalisches Institut, Aachen, Germany

C. Autermann, L. Feld, M.K. Kiesel, K. Klein, M. Lipinski, D. Meuser, A. Pauls, M. Preuten, M.P. Rauch, C. Schomakers, J. Schulz, M. Teroerde, B. Wittmer

RWTH Aachen University, III. Physikalisches Institut A, Aachen, Germany

A. Albert, M. Erdmann, S. Erdweg, T. Esch, B. Fischer, R. Fischer, S. Ghosh, T. Hebbeker, K. Hoepfner, H. Keller, L. Mastrolorenzo, M. Merschmeyer, A. Meyer, P. Millet, G. Mocellin, S. Mondal, S. Mukherjee, D. Noll, A. Novak, T. Pook, A. Pozdnyakov, T. Quast, M. Radziej, Y. Rath, H. Reithler, M. Rieger, J. Roemer, A. Schmidt, S.C. Schuler, A. Sharma, S. Thüer, S. Wiedenbeck

RWTH Aachen University, III. Physikalisches Institut B, Aachen, Germany G. Flügge, W. Haj Ahmad ${ }^{15}$, O. Hlushchenko, T. Kress, T. Müller, A. Nehrkorn, A. Nowack, C. Pistone, O. Pooth, D. Roy, H. Sert, A. Stahl ${ }^{16}$

Deutsches Elektronen-Synchrotron, Hamburg, Germany

M. Aldaya Martin, P. Asmuss, I. Babounikau, H. Bakhshiansohi, K. Beernaert, O. Behnke, U. Behrens, A. Bermúdez Martínez, D. Bertsche, A.A. Bin Anuar, K. Borras ${ }^{17}$, V. Botta, A. Campbell, A. Cardini, P. Connor, S. Consuegra Rodríguez, C. Contreras-Campana, V. Danilov, A. De Wit, M.M. Defranchis, C. Diez Pardos, D. Domínguez Damiani, G. Eckerlin, D. Eckstein, T. Eichhorn, A. Elwood, E. Eren, E. Gallo ${ }^{18}$, A. Geiser, J.M. Grados Luyando, A. Grohsjean, M. Guthoff, M. Haranko, A. Harb, A. Jafari, N.Z. Jomhari, H. Jung, A. Kasem ${ }^{17}$, M. Kasemann, H. Kaveh, J. Keaveney, C. Kleinwort, J. Knolle, D. Krücker, W. Lange, T. Lenz, J. Leonard, J. Lidrych, K. Lipka, W. Lohmann ${ }^{19}$, R. Mankel, I.-A. Melzer-Pellmann, A.B. Meyer, M. Meyer, M. Missiroli, G. Mittag, J. Mnich, A. Mussgiller, V. Myronenko, D. Pérez Adán, S.K. Pflitsch, D. Pitzl, A. Raspereza, A. Saibel, M. Savitskyi, V. Scheurer, P. Schütze, C. Schwanenberger, R. Shevchenko, A. Singh, H. Tholen, O. Turkot, A. Vagnerini, M. Van De Klundert, G.P. Van Onsem, R. Walsh, Y. Wen, K. Wichmann, C. Wissing, O. Zenaiev, R. Zlebcik

University of Hamburg, Hamburg, Germany

R. Aggleton, S. Bein, L. Benato, A. Benecke, V. Blobel, T. Dreyer, A. Ebrahimi, A. Fröhlich, C. Garbers, E. Garutti, D. Gonzalez, P. Gunnellini, J. Haller, A. Hinzmann, A. Karavdina, G. Kasieczka, R. Klanner, R. Kogler, N. Kovalchuk, S. Kurz, V. Kutzner, 
J. Lange, T. Lange, A. Malara, D. Marconi, J. Multhaup, M. Niedziela, C.E.N. Niemeyer, D. Nowatschin, A. Perieanu, A. Reimers, O. Rieger, C. Scharf, P. Schleper, S. Schumann, J. Schwandt, J. Sonneveld, H. Stadie, G. Steinbrück, F.M. Stober, M. Stöver, B. Vormwald, I. Zoi

Karlsruher Institut fuer Technologie, Karlsruhe, Germany

M. Akbiyik, C. Barth, M. Baselga, S. Baur, T. Berger, E. Butz, R. Caspart, T. Chwalek, W. De Boer, A. Dierlamm, K. El Morabit, N. Faltermann, M. Giffels, P. Goldenzweig, A. Gottmann, M.A. Harrendorf, F. Hartmann ${ }^{16}$, U. Husemann, S. Kudella, S. Mitra, M.U. Mozer, Th. Müller, M. Musich, A. Nürnberg, G. Quast, K. Rabbertz, M. Schröder, I. Shvetsov, H.J. Simonis, R. Ulrich, M. Weber, C. Wöhrmann, R. Wolf

Institute of Nuclear and Particle Physics (INPP), NCSR Demokritos, Aghia Paraskevi, Greece

G. Anagnostou, P. Asenov, G. Daskalakis, T. Geralis, A. Kyriakis, D. Loukas, G. Paspalaki National and Kapodistrian University of Athens, Athens, Greece

M. Diamantopoulou, G. Karathanasis, P. Kontaxakis, A. Panagiotou, I. Papavergou, N. Saoulidou, A. Stakia, K. Theofilatos, K. Vellidis

National Technical University of Athens, Athens, Greece

G. Bakas, K. Kousouris, I. Papakrivopoulos, G. Tsipolitis

University of Ioánnina, Ioánnina, Greece

I. Evangelou, C. Foudas, P. Gianneios, P. Katsoulis, P. Kokkas, S. Mallios, K. Manitara, N. Manthos, I. Papadopoulos, J. Strologas, F.A. Triantis, D. Tsitsonis

MTA-ELTE Lendület CMS Particle and Nuclear Physics Group, Eötvös Loránd University, Budapest, Hungary

M. Bartók ${ }^{20}$, M. Csanad, P. Major, K. Mandal, A. Mehta, M.I. Nagy, G. Pasztor, O. Surányi, G.I. Veres

Wigner Research Centre for Physics, Budapest, Hungary

G. Bencze, C. Hajdu, D. Horvath ${ }^{21}$, F. Sikler, T.Á. Vámi, V. Veszpremi, G. Vesztergombi ${ }^{\dagger}$

Institute of Nuclear Research ATOMKI, Debrecen, Hungary

N. Beni, S. Czellar, J. Karancsi ${ }^{20}$, A. Makovec, J. Molnar, Z. Szillasi

Institute of Physics, University of Debrecen, Debrecen, Hungary

P. Raics, D. Teyssier, Z.L. Trocsanyi, B. Ujvari

Eszterhazy Karoly University, Karoly Robert Campus, Gyongyos, Hungary

T. Csorgo, W.J. Metzger, F. Nemes, T. Novak

Indian Institute of Science (IISc), Bangalore, India

S. Choudhury, J.R. Komaragiri, P.C. Tiwari 
National Institute of Science Education and Research, HBNI, Bhubaneswar, India

S. Bahinipati ${ }^{23}$, C. Kar, P. Mal, V.K. Muraleedharan Nair Bindhu, A. Nayak ${ }^{24}$, D.K. Sahoo ${ }^{23}$, S.K. Swain

Panjab University, Chandigarh, India

S. Bansal, S.B. Beri, V. Bhatnagar, S. Chauhan, R. Chawla, N. Dhingra, R. Gupta,

A. Kaur, M. Kaur, S. Kaur, P. Kumari, M. Lohan, M. Meena, K. Sandeep, S. Sharma, J.B. Singh, A.K. Virdi, G. Walia

University of Delhi, Delhi, India

A. Bhardwaj, B.C. Choudhary, R.B. Garg, M. Gola, S. Keshri, Ashok Kumar, S. Malhotra, M. Naimuddin, P. Priyanka, K. Ranjan, Aashaq Shah, R. Sharma

Saha Institute of Nuclear Physics, HBNI, Kolkata, India

R. Bhardwaj ${ }^{25}$, M. Bharti ${ }^{25}$, R. Bhattacharya, S. Bhattacharya, U. Bhawandeep ${ }^{25}$, D. Bhowmik, S. Dey, S. Dutta, S. Ghosh, M. Maity ${ }^{26}$, K. Mondal, S. Nandan, A. Purohit, P.K. Rout, A. Roy, G. Saha, S. Sarkar, T. Sarkar ${ }^{26}$, M. Sharan, B. Singh ${ }^{25}$, S. Thakur ${ }^{25}$

Indian Institute of Technology Madras, Madras, India

P.K. Behera, P. Kalbhor, A. Muhammad, P.R. Pujahari, A. Sharma, A.K. Sikdar

Bhabha Atomic Research Centre, Mumbai, India

R. Chudasama, D. Dutta, V. Jha, V. Kumar, D.K. Mishra, P.K. Netrakanti, L.M. Pant, P. Shukla

Tata Institute of Fundamental Research-A, Mumbai, India

T. Aziz, M.A. Bhat, S. Dugad, G.B. Mohanty, N. Sur, RavindraKumar Verma

Tata Institute of Fundamental Research-B, Mumbai, India

S. Banerjee, S. Bhattacharya, S. Chatterjee, P. Das, M. Guchait, S. Karmakar, S. Kumar, G. Majumder, K. Mazumdar, N. Sahoo, S. Sawant

Indian Institute of Science Education and Research (IISER), Pune, India

S. Chauhan, S. Dube, V. Hegde, A. Kapoor, K. Kothekar, S. Pandey, A. Rane, A. Rastogi,

S. Sharma

Institute for Research in Fundamental Sciences (IPM), Tehran, Iran

S. Chenarani ${ }^{27}$, E. Eskandari Tadavani, S.M. Etesami ${ }^{27}$, M. Khakzad, M. Mohammadi Najafabadi, M. Naseri, F. Rezaei Hosseinabadi

University College Dublin, Dublin, Ireland

M. Felcini, M. Grunewald

INFN Sezione di Bari ${ }^{a}$, Università di Bari ${ }^{b}$, Politecnico di Bari ${ }^{c}$, Bari, Italy

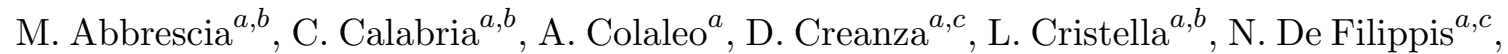
M. De Palma ${ }^{a, b}$, A. Di Florio ${ }^{a, b}$, L. Fiore $^{a}$, A. Gelmi ${ }^{a, b}$, G. Iaselli $^{a, c}$, M. Ince $^{a, b}$, S. Lezki $^{a, b}$, G. Maggi ${ }^{a, c}$, M. Maggi ${ }^{a}$, G. Miniello ${ }^{a, b}$, S. My ${ }^{a, b}$, S. Nuzzo $^{a, b}$, A. Pompili ${ }^{a, b}$, G. Pugliese ${ }^{a, c}$, R. Radogna ${ }^{a}$, A. Ranieri ${ }^{a}$, G. Selvaggi ${ }^{a}, b$, L. Silvestris ${ }^{a}$, R. Venditti ${ }^{a}$, P. Verwilligen $^{a}$ 
INFN Sezione di Bologna ${ }^{a}$, Università di Bologna ${ }^{b}$, Bologna, Italy

G. Abbiendi ${ }^{a}$, C. Battilana ${ }^{a, b}$, D. Bonacorsi ${ }^{a, b}$, L. Borgonovi $^{a, b}$, S. Braibant-Giacomelli $^{a, b}$, R. Campanini ${ }^{a, b}$, P. Capiluppi ${ }^{a, b}$, A. Castro ${ }^{a, b}$, F.R. Cavallo ${ }^{a}$, C. Ciocca ${ }^{a}$, G. Codispoti $^{a, b}$, M. Cuffiani ${ }^{a, b}$, G.M. Dallavalle ${ }^{a}$, F. Fabbri ${ }^{a}$, A. Fanfani ${ }^{a, b}$, E. Fontanesi, P. Giacomelli ${ }^{a}$, C. Grandi ${ }^{a}$, L. Guiducci ${ }^{a, b}$, F. Iemmi ${ }^{a, b}$, S. Lo Meo ${ }^{a, 28}$, S. Marcellini ${ }^{a}$, G. Masetti ${ }^{a}$,

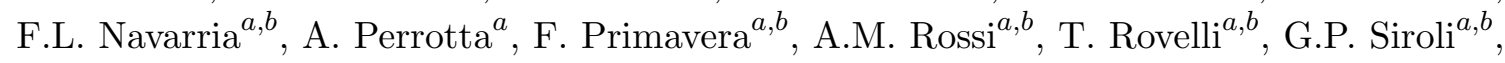
N. $\operatorname{Tosi}^{a}$

INFN Sezione di Catania ${ }^{a}$, Università di Catania ${ }^{b}$, Catania, Italy

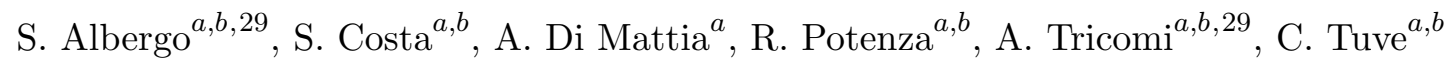

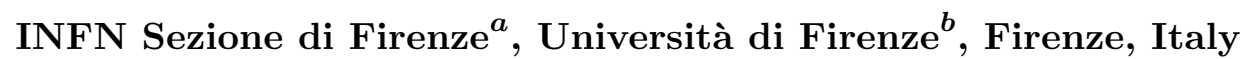

G. Barbagli ${ }^{a}$, R. Ceccarelli, K. Chatterjee ${ }^{a, b}$, V. Ciulli ${ }^{a, b}$, C. Civinini ${ }^{a}$, R. D'Alessandro ${ }^{a, b}{ }^{,}$ E. Focardi ${ }^{a, b}$, G. Latino, P. Lenzi ${ }^{a, b}$, M. Meschini $^{a}$, S. Paoletti $^{a}$, G. Sguazzoni ${ }^{a}$, D. Strom ${ }^{a}$, L. Viliani ${ }^{a}$

INFN Laboratori Nazionali di Frascati, Frascati, Italy

L. Benussi, S. Bianco, D. Piccolo

INFN Sezione di Genova ${ }^{a}$, Università di Genova ${ }^{b}$, Genova, Italy

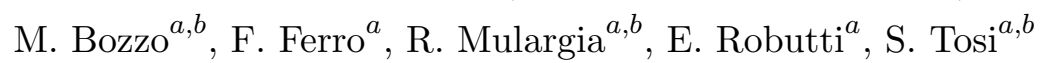

INFN Sezione di Milano-Bicocca $^{a}$, Università di Milano-Bicocca ${ }^{b}$, Milano, Italy

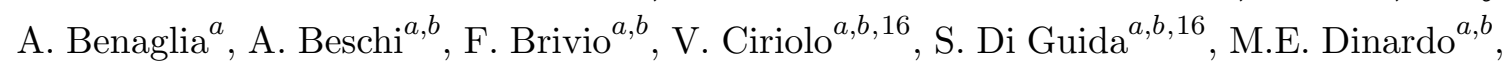

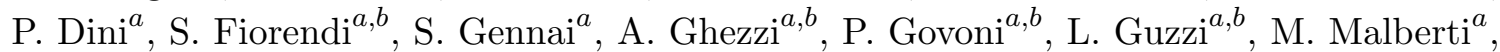
S. Malvezzi ${ }^{a}$, D. Menasce ${ }^{a}$, F. Monti ${ }^{a, b}$, L. Moroni ${ }^{a}$, G. Ortona ${ }^{a, b}$, M. Paganoni $^{a, b}$, D. Pedrini $^{a}$, S. Ragazzi ${ }^{a, b}$, T. Tabarelli de Fatis ${ }^{a, b}$, D. Zuolo ${ }^{a, b}$

INFN Sezione di Napoli ${ }^{a}$, Università di Napoli 'Federico II'b , Napoli, Italy, Università della Basilicata $^{c}$, Potenza, Italy, Università G. Marconi ${ }^{d}$, Roma, Italy

S. Buontempo ${ }^{a}$, N. Cavallo $^{a, c}$, A. De Iorio ${ }^{a, b}$, A. Di Crescenzo ${ }^{a, b}$, F. Fabozzi $^{a, c}$, F. Fienga $^{a}$,

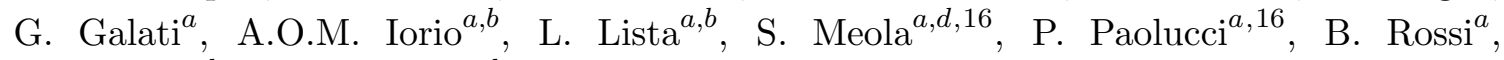
C. Sciacca ${ }^{a, b}$, E. Voevodina ${ }^{a, b}$

INFN Sezione di Padova ${ }^{a}$, Università di Padova ${ }^{b}$, Padova, Italy, Università di Trento $^{c}$, Trento, Italy

P. Azzi ${ }^{a}$, N. Bacchetta ${ }^{a}$, A. Boletti ${ }^{a, b}$, A. Bragagnolo, R. Carlin ${ }^{a, b}$, P. Checchia ${ }^{a}$, P. De Castro Manzano $^{a}$, T. Dorigo ${ }^{a}$, U. Dosselli ${ }^{a}$, F. Gasparini ${ }^{a, b}$, U. Gasparini ${ }^{a, b}$, A. Gozzelino $^{a}$, S.Y. Hoh, P. Lujan, M. Margoni ${ }^{a, b}$, A.T. Meneguzzo ${ }^{a, b}$, J. Pazzini ${ }^{a, b}$, N. Pozzobon ${ }^{a, b}$, M. Presilla ${ }^{b}$, P. Ronchese ${ }^{a, b}$, R. Rossin ${ }^{a, b}$, F. Simonetto ${ }^{a, b}$, A. Tiko, M. Tosi ${ }^{a, b}$, M. Zanetti ${ }^{a, b}$, P. Zotto ${ }^{a, b}$, G. Zumerle ${ }^{a, b}$

INFN Sezione di Pavia ${ }^{a}$, Università di Pavia ${ }^{b}$, Pavia, Italy

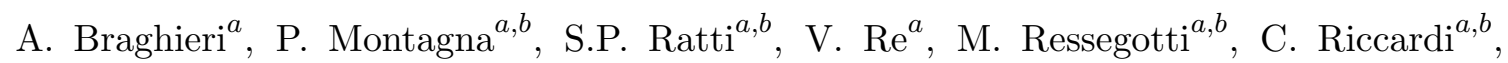
P. Salvini ${ }^{a}$, I. Vai ${ }^{a, b}$, P. Vitulo ${ }^{a, b}$ 
INFN Sezione di Perugia ${ }^{a}$, Università di Perugia ${ }^{b}$, Perugia, Italy

M. Biasini ${ }^{a, b}$, G.M. Bilei ${ }^{a}$, C. Cecchi ${ }^{a, b}$, D. Ciangottini ${ }^{a, b}$, L. Fanò ${ }^{a, b}$, P. Lariccia ${ }^{a, b}$,

R. Leonardi ${ }^{a, b}$, E. Manoni ${ }^{a}$, G. Mantovani ${ }^{a, b}$, V. Mariani ${ }^{a, b}$, M. Menichelli $^{a}$, A. Rossi $^{a, b}$,

A. Santocchia ${ }^{a, b}$, D. Spiga ${ }^{a}$

INFN Sezione di Pisa ${ }^{a}$, Università di Pisa ${ }^{b}$, Scuola Normale Superiore di Pisa ${ }^{c}$, Pisa, Italy

K. $\operatorname{Androsov}^{a}$, P. Azzurri ${ }^{a}$, G. Bagliesi ${ }^{a}$, V. Bertacchi ${ }^{a, c}$, L. Bianchini ${ }^{a}$, T. Boccali ${ }^{a}$,

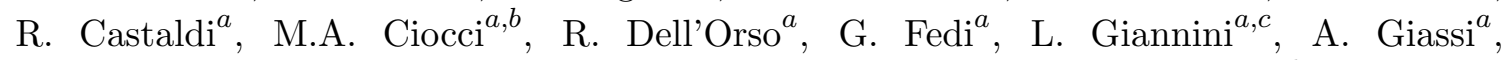
M.T. Grippo ${ }^{a}$, F. Ligabue ${ }^{a, c}$, E. Manca ${ }^{a, c}$, G. Mandorli ${ }^{a, c}$, A. Messineo ${ }^{a, b}$, F. Palla $^{a}$, A. Rizzi ${ }^{a, b}$, G. Rolandi ${ }^{30}$, S. Roy Chowdhury, A. Scribano ${ }^{a}$, P. Spagnolo $^{a}$, R. Tenchini $^{a}$, G. Tonelli ${ }^{a, b}$, N. Turini, A. Venturi ${ }^{a}$, P.G. Verdini ${ }^{a}$

INFN Sezione di Roma ${ }^{a}$, Sapienza Università di Roma ${ }^{b}$, Rome, Italy

F. Cavallari ${ }^{a}$, M. Cipriani ${ }^{a, b}$, D. Del Re ${ }^{a, b}$, E. Di Marco ${ }^{a, b}$, M. Diemoz $^{a}$, E. Longo ${ }^{a, b}$,

B. Marzocchi $^{a, b}$, P. Meridiani ${ }^{a}$, G. Organtini ${ }^{a, b}$, F. Pandolfi ${ }^{a}$, R. Paramatti ${ }^{a, b}$,

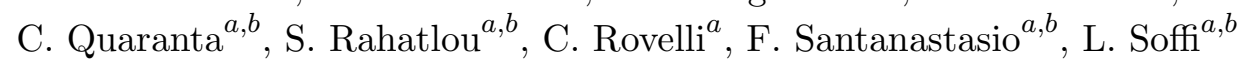

INFN Sezione di Torino ${ }^{a}$, Università di Torino ${ }^{b}$, Torino, Italy, Università del Piemonte Orientale ${ }^{c}$, Novara, Italy

N. Amapane ${ }^{a, b}$, R. Arcidiacono ${ }^{a, c}, \mathrm{~S}$. Argiro ${ }^{a, b}$, M. Arneodo $^{a, c}$, N. Bartosik ${ }^{a}$, R. Bellan $^{a, b}$,

C. Biino ${ }^{a}$, A. Cappati ${ }^{a, b}$, N. Cartiglia $^{a}$, S. Cometti ${ }^{a}$, M. Costa ${ }^{a, b}$, R. Covarelli ${ }^{a, b}$,

N. Demaria ${ }^{a}$, B. Kiani ${ }^{a, b}$, C. Mariotti $^{a}$, S. Maselli ${ }^{a}$, E. Migliore ${ }^{a, b}$, V. Monaco ${ }^{a, b}$,

E. Monteil ${ }^{a, b}$, M. Monteno $^{a}$, M.M. Obertino ${ }^{a, b}$, L. Pacher $^{a, b}$, N. Pastrone $^{a}$, M. Pelliccioni ${ }^{a}$, G.L. Pinna Angioni ${ }^{a, b}$, A. Romero ${ }^{a, b}$, M. Ruspa $^{a, c}$, R. Sacchi ${ }^{a, b}$, R. Salvatico $^{a, b}$, V. Sola $^{a}$, A. Solano ${ }^{a, b}$, D. Soldi ${ }^{a, b}$, A. Staiano ${ }^{a}$

INFN Sezione di Trieste ${ }^{a}$, Università di Trieste ${ }^{b}$, Trieste, Italy

S. Belforte ${ }^{a}$, V. Candelise ${ }^{a, b}$, M. Casarsa ${ }^{a}$, F. Cossutti ${ }^{a}$, A. Da Rold ${ }^{a, b}$, G. Della Ricca ${ }^{a, b}$, F. Vazzoler ${ }^{a, b}$, A. Zanetti ${ }^{a}$

Kyungpook National University, Daegu, Korea

B. Kim, D.H. Kim, G.N. Kim, M.S. Kim, J. Lee, S.W. Lee, C.S. Moon, Y.D. Oh, S.I. Pak,

S. Sekmen, D.C. Son, Y.C. Yang

Chonnam National University, Institute for Universe and Elementary Particles, Kwangju, Korea

H. Kim, D.H. Moon, G. Oh

Hanyang University, Seoul, Korea

B. Francois, T.J. Kim, J. Park

Korea University, Seoul, Korea

S. Cho, S. Choi, Y. Go, D. Gyun, S. Ha, B. Hong, K. Lee, K.S. Lee, J. Lim, J. Park, S.K. Park, Y. Roh

Kyung Hee University, Department of Physics

J. Goh 
Sejong University, Seoul, Korea

H.S. Kim

Seoul National University, Seoul, Korea

J. Almond, J.H. Bhyun, J. Choi, S. Jeon, J. Kim, J.S. Kim, H. Lee, K. Lee, S. Lee, K. Nam, M. Oh, S.B. Oh, B.C. Radburn-Smith, U.K. Yang, H.D. Yoo, I. Yoon, G.B. Yu

University of Seoul, Seoul, Korea

D. Jeon, H. Kim, J.H. Kim, J.S.H. Lee, I.C. Park, I. Watson

Sungkyunkwan University, Suwon, Korea

Y. Choi, C. Hwang, Y. Jeong, J. Lee, Y. Lee, I. Yu

Riga Technical University, Riga, Latvia

V. Veckalns ${ }^{31}$

Vilnius University, Vilnius, Lithuania

V. Dudenas, A. Juodagalvis, J. Vaitkus

National Centre for Particle Physics, Universiti Malaya, Kuala Lumpur, Malaysia

Z.A. Ibrahim, F. Mohamad Idris ${ }^{32}$, W.A.T. Wan Abdullah, M.N. Yusli, Z. Zolkapli

Universidad de Sonora (UNISON), Hermosillo, Mexico

J.F. Benitez, A. Castaneda Hernandez, J.A. Murillo Quijada, L. Valencia Palomo

Centro de Investigacion y de Estudios Avanzados del IPN, Mexico City, Mexico

H. Castilla-Valdez, E. De La Cruz-Burelo, I. Heredia-De La Cruz ${ }^{33}$, R. Lopez-Fernandez,

A. Sanchez-Hernandez

Universidad Iberoamericana, Mexico City, Mexico

S. Carrillo Moreno, C. Oropeza Barrera, M. Ramirez-Garcia, F. Vazquez Valencia

Benemerita Universidad Autonoma de Puebla, Puebla, Mexico

J. Eysermans, I. Pedraza, H.A. Salazar Ibarguen, C. Uribe Estrada

Universidad Autónoma de San Luis Potosí, San Luis Potosí, Mexico

A. Morelos Pineda

University of Montenegro, Podgorica, Montenegro

N. Raicevic

University of Auckland, Auckland, New Zealand

D. Krofcheck

University of Canterbury, Christchurch, New Zealand

S. Bheesette, P.H. Butler

National Centre for Physics, Quaid-I-Azam University, Islamabad, Pakistan

A. Ahmad, M. Ahmad, Q. Hassan, H.R. Hoorani, W.A. Khan, M.A. Shah, M. Shoaib, M. Waqas 
AGH University of Science and Technology Faculty of Computer Science, Electronics and Telecommunications, Krakow, Poland

V. Avati, L. Grzanka, M. Malawski

National Centre for Nuclear Research, Swierk, Poland

H. Bialkowska, M. Bluj, B. Boimska, M. Górski, M. Kazana, M. Szleper, P. Zalewski

Institute of Experimental Physics, Faculty of Physics, University of Warsaw, Warsaw, Poland

K. Bunkowski, A. Byszuk ${ }^{34}$, K. Doroba, A. Kalinowski, M. Konecki, J. Krolikowski, M. Misiura, M. Olszewski, A. Pyskir, M. Walczak

Laboratório de Instrumentação e Física Experimental de Partículas, Lisboa, Portugal

M. Araujo, P. Bargassa, D. Bastos, A. Di Francesco, P. Faccioli, B. Galinhas, M. Gallinaro, J. Hollar, N. Leonardo, J. Seixas, K. Shchelina, G. Strong, O. Toldaiev, J. Varela

Joint Institute for Nuclear Research, Dubna, Russia

S. Afanasiev, P. Bunin, M. Gavrilenko, I. Golutvin, I. Gorbunov, A. Kamenev, V. Karjavine, A. Lanev, A. Malakhov, V. Matveev ${ }^{35,36}$, P. Moisenz, V. Palichik, V. Perelygin, M. Savina, S. Shmatov, S. Shulha, N. Skatchkov, V. Smirnov, N. Voytishin, A. Zarubin

Petersburg Nuclear Physics Institute, Gatchina (St. Petersburg), Russia

L. Chtchipounov, V. Golovtsov, Y. Ivanov, V. Kim ${ }^{37}$, E. Kuznetsova ${ }^{38}$, P. Levchenko, V. Murzin, V. Oreshkin, I. Smirnov, D. Sosnov, V. Sulimov, L. Uvarov, A. Vorobyev

Institute for Nuclear Research, Moscow, Russia

Yu. Andreev, A. Dermenev, S. Gninenko, N. Golubev, A. Karneyeu, M. Kirsanov, N. Krasnikov, A. Pashenkov, D. Tlisov, A. Toropin

Institute for Theoretical and Experimental Physics named by A.I. Alikhanov of NRC 'Kurchatov Institute', Moscow, Russia

V. Epshteyn, V. Gavrilov, N. Lychkovskaya, A. Nikitenko ${ }^{39}$, V. Popov, I. Pozdnyakov, G. Safronov, A. Spiridonov, A. Stepennov, M. Toms, E. Vlasov, A. Zhokin

Moscow Institute of Physics and Technology, Moscow, Russia

T. Aushev

National Research Nuclear University 'Moscow Engineering Physics Institute' (MEPhI), Moscow, Russia

O. Bychkova, R. Chistov ${ }^{40}$, M. Danilov ${ }^{40}$, S. Polikarpov ${ }^{40}$, E. Tarkovskii

P.N. Lebedev Physical Institute, Moscow, Russia

V. Andreev, M. Azarkin, I. Dremin, M. Kirakosyan, A. Terkulov

Skobeltsyn Institute of Nuclear Physics, Lomonosov Moscow State University, Moscow, Russia

A. Baskakov, A. Belyaev, E. Boos, V. Bunichev, M. Dubinin ${ }^{41}$, L. Dudko, V. Klyukhin, N. Korneeva, I. Lokhtin, S. Obraztsov, M. Perfilov, V. Savrin, P. Volkov 
Novosibirsk State University (NSU), Novosibirsk, Russia

A. Barnyakov ${ }^{42}$, V. Blinov ${ }^{42}$, T. Dimova ${ }^{42}$, L. Kardapoltsev ${ }^{42}$, Y. Skovpen ${ }^{42}$

Institute for High Energy Physics of National Research Centre 'Kurchatov Institute', Protvino, Russia

I. Azhgirey, I. Bayshev, S. Bitioukov, V. Kachanov, D. Konstantinov, P. Mandrik, V. Petrov, R. Ryutin, S. Slabospitskii, A. Sobol, S. Troshin, N. Tyurin, A. Uzunian, A. Volkov

National Research Tomsk Polytechnic University, Tomsk, Russia

A. Babaev, A. Iuzhakov, V. Okhotnikov

Tomsk State University, Tomsk, Russia

V. Borchsh, V. Ivanchenko, E. Tcherniaev

University of Belgrade: Faculty of Physics and VINCA Institute of Nuclear Sciences

P. Adzic ${ }^{43}$, P. Cirkovic, D. Devetak, M. Dordevic, P. Milenovic, J. Milosevic, M. Stojanovic

Centro de Investigaciones Energéticas Medioambientales y Tecnológicas (CIEMAT), Madrid, Spain

M. Aguilar-Benitez, J. Alcaraz Maestre, A. Álvarez Fernández, I. Bachiller, M. Barrio Luna, J.A. Brochero Cifuentes, C.A. Carrillo Montoya, M. Cepeda, M. Cerrada, N. Colino, B. De La Cruz, A. Delgado Peris, C. Fernandez Bedoya, J.P. Fernández Ramos, J. Flix, M.C. Fouz, O. Gonzalez Lopez, S. Goy Lopez, J.M. Hernandez, M.I. Josa, D. Moran, Á. Navarro Tobar, A. Pérez-Calero Yzquierdo, J. Puerta Pelayo, I. Redondo, L. Romero, S. Sánchez Navas, M.S. Soares, A. Triossi, C. Willmott

Universidad Autónoma de Madrid, Madrid, Spain

C. Albajar, J.F. de Trocóniz

Universidad de Oviedo, Oviedo, Spain

B. Alvarez Gonzalez, J. Cuevas, C. Erice, J. Fernandez Menendez, S. Folgueras, I. Gonzalez Caballero, J.R. González Fernández, E. Palencia Cortezon, V. Rodríguez Bouza, S. Sanchez Cruz

Instituto de Física de Cantabria (IFCA), CSIC-Universidad de Cantabria, Santander, Spain

I.J. Cabrillo, A. Calderon, B. Chazin Quero, J. Duarte Campderros, M. Fernandez, P.J. Fernández Manteca, A. García Alonso, G. Gomez, C. Martinez Rivero, P. Martinez Ruiz del Arbol, F. Matorras, J. Piedra Gomez, C. Prieels, T. Rodrigo, A. Ruiz-Jimeno, L. Russo $^{44}$, L. Scodellaro, N. Trevisani, I. Vila, J.M. Vizan Garcia

University of Colombo, Colombo, Sri Lanka

K. Malagalage

University of Ruhuna, Department of Physics, Matara, Sri Lanka

W.G.D. Dharmaratna, N. Wickramage 


\section{CERN, European Organization for Nuclear Research, Geneva, Switzerland}

D. Abbaneo, B. Akgun, E. Auffray, G. Auzinger, J. Baechler, P. Baillon, A.H. Ball, D. Barney, J. Bendavid, M. Bianco, A. Bocci, E. Bossini, C. Botta, E. Brondolin, T. Camporesi, A. Caratelli, G. Cerminara, E. Chapon, G. Cucciati, D. d'Enterria, A. Dabrowski, N. Daci, V. Daponte, A. David, O. Davignon, A. De Roeck, N. Deelen, M. Deile, M. Dobson, M. Dünser, N. Dupont, A. Elliott-Peisert, F. Fallavollita ${ }^{45}$, D. Fasanella, G. Franzoni, J. Fulcher, W. Funk, S. Giani, D. Gigi, A. Gilbert, K. Gill, F. Glege, M. Gruchala, M. Guilbaud, D. Gulhan, J. Hegeman, C. Heidegger, Y. Iiyama, V. Innocente, P. Janot, O. Karacheban ${ }^{19}$, J. Kaspar, J. Kieseler, M. Krammer ${ }^{1}$, C. Lange, P. Lecoq, C. Lourenço, L. Malgeri, M. Mannelli, A. Massironi, F. Meijers, J.A. Merlin, S. Mersi, E. Meschi, F. Moortgat, M. Mulders, J. Ngadiuba, S. Nourbakhsh, S. Orfanelli, L. Orsini, F. Pantaleo ${ }^{16}$, L. Pape, E. Perez, M. Peruzzi, A. Petrilli, G. Petrucciani, A. Pfeiffer, M. Pierini, F.M. Pitters, D. Rabady, A. Racz, M. Rovere, H. Sakulin, C. Schäfer, C. Schwick, M. Selvaggi, A. Sharma, P. Silva, W. Snoeys, P. Sphicas ${ }^{46}$, J. Steggemann, V.R. Tavolaro, D. Treille, A. Tsirou, A. Vartak, M. Verzetti, W.D. Zeuner

\section{Paul Scherrer Institut, Villigen, Switzerland}

L. Caminada ${ }^{47}$, K. Deiters, W. Erdmann, R. Horisberger, Q. Ingram, H.C. Kaestli, D. Kotlinski, U. Langenegger, T. Rohe, S.A. Wiederkehr

ETH Zurich - Institute for Particle Physics and Astrophysics (IPA), Zurich, Switzerland

M. Backhaus, P. Berger, N. Chernyavskaya, G. Dissertori, M. Dittmar, M. Donegà, C. Dorfer, T.A. Gómez Espinosa, C. Grab, D. Hits, T. Klijnsma, W. Lustermann, R.A. Manzoni, M. Marionneau, M.T. Meinhard, F. Micheli, P. Musella, F. Nessi-Tedaldi, F. Pauss, G. Perrin, L. Perrozzi, S. Pigazzini, M. Reichmann, C. Reissel, T. Reitenspiess, D. Ruini, D.A. Sanz Becerra, M. Schönenberger, L. Shchutska, M.L. Vesterbacka Olsson, R. Wallny, D.H. Zhu

\section{Universität Zürich, Zurich, Switzerland}

T.K. Aarrestad, C. Amsler ${ }^{48}$, D. Brzhechko, M.F. Canelli, A. De Cosa, R. Del Burgo, S. Donato, B. Kilminster, S. Leontsinis, V.M. Mikuni, I. Neutelings, G. Rauco, P. Robmann, D. Salerno, K. Schweiger, C. Seitz, Y. Takahashi, S. Wertz, A. Zucchetta

\section{National Central University, Chung-Li, Taiwan}

T.H. Doan, C.M. Kuo, W. Lin, S.S. Yu

\section{National Taiwan University (NTU), Taipei, Taiwan}

P. Chang, Y. Chao, K.F. Chen, P.H. Chen, W.-S. Hou, Y.y. Li, R.-S. Lu, E. Paganis, A. Psallidas, A. Steen

Chulalongkorn University, Faculty of Science, Department of Physics, Bangkok, Thailand

B. Asavapibhop, C. Asawatangtrakuldee, N. Srimanobhas, N. Suwonjandee, V. Wachirapusitanand 
Çukurova University, Physics Department, Science and Art Faculty, Adana, Turkey

A. Bat, F. Boran, S. Cerci $^{49}$, S. Damarseckin ${ }^{50}$, Z.S. Demiroglu, F. Dolek, C. Dozen, I. Dumanoglu, G. Gokbulut, EmineGurpinar Guler ${ }^{51}$, Y. Guler, I. Hos ${ }^{52}$, C. Isik, E.E. Kangal ${ }^{53}$, O. Kara, A. Kayis Topaksu, U. Kiminsu, M. Oglakci, G. Onengut, K. Ozdemir ${ }^{54}$, S. Ozturk ${ }^{55}$, A.E. Simsek, D. Sunar Cerci ${ }^{49}$, U.G. Tok, S. Turkcapar, I.S. Zorbakir, C. Zorbilmez

Middle East Technical University, Physics Department, Ankara, Turkey B. Isildak ${ }^{56}$, G. Karapinar $^{57}$, M. Yalvac

Bogazici University, Istanbul, Turkey

I.O. Atakisi, E. Gülmez, O. Kaya ${ }^{58}$, B. Kaynak, Ö. Özçelik, S. Ozkorucuklu ${ }^{59}$, S. Tekten, E.A. Yetkin ${ }^{60}$

Istanbul Technical University, Istanbul, Turkey

A. Cakir, Y. Komurcu, S. Sen ${ }^{61}$

Institute for Scintillation Materials of National Academy of Science of Ukraine, Kharkov, Ukraine

B. Grynyov

National Scientific Center, Kharkov Institute of Physics and Technology, Kharkov, Ukraine

L. Levchuk

University of Bristol, Bristol, United Kingdom

F. Ball, E. Bhal, S. Bologna, J.J. Brooke, D. Burns, E. Clement, D. Cussans, H. Flacher, J. Goldstein, G.P. Heath, H.F. Heath, L. Kreczko, S. Paramesvaran, B. Penning, T. Sakuma, S. Seif El Nasr-Storey, D. Smith, V.J. Smith, J. Taylor, A. Titterton

\section{Rutherford Appleton Laboratory, Didcot, United Kingdom}

K.W. Bell, A. Belyaev ${ }^{62}$, C. Brew, R.M. Brown, D. Cieri, D.J.A. Cockerill, J.A. Coughlan, K. Harder, S. Harper, J. Linacre, K. Manolopoulos, D.M. Newbold, E. Olaiya, D. Petyt, T. Reis, T. Schuh, C.H. Shepherd-Themistocleous, A. Thea, I.R. Tomalin, T. Williams, W.J. Womersley

\section{Imperial College, London, United Kingdom}

R. Bainbridge, P. Bloch, J. Borg, S. Breeze, O. Buchmuller, A. Bundock, GurpreetSingh CHAHAL ${ }^{63}$, D. Colling, P. Dauncey, G. Davies, M. Della Negra, R. Di Maria, P. Everaerts, G. Hall, G. Iles, T. James, M. Komm, C. Laner, L. Lyons, A.-M. Magnan, S. Malik, A. Martelli, V. Milosevic, J. Nash ${ }^{64}$, V. Palladino, M. Pesaresi, D.M. Raymond, A. Richards, A. Rose, E. Scott, C. Seez, A. Shtipliyski, M. Stoye, T. Strebler, S. Summers, A. Tapper, K. Uchida, T. Virdee ${ }^{16}$, N. Wardle, D. Winterbottom, J. Wright, A.G. Zecchinelli, S.C. Zenz 
Brunel University, Uxbridge, United Kingdom

J.E. Cole, P.R. Hobson, A. Khan, P. Kyberd, C.K. Mackay, A. Morton, I.D. Reid, L. Teodorescu, S. Zahid

Baylor University, Waco, U.S.A.

K. Call, J. Dittmann, K. Hatakeyama, C. Madrid, B. McMaster, N. Pastika, C. Smith

Catholic University of America, Washington, DC, U.S.A.

R. Bartek, A. Dominguez, R. Uniyal

The University of Alabama, Tuscaloosa, U.S.A.

A. Buccilli, S.I. Cooper, C. Henderson, P. Rumerio, C. West

Boston University, Boston, U.S.A.

D. Arcaro, T. Bose, Z. Demiragli, D. Gastler, S. Girgis, D. Pinna, C. Richardson, J. Rohlf,

D. Sperka, I. Suarez, L. Sulak, D. Zou

Brown University, Providence, U.S.A.

G. Benelli, B. Burkle, X. Coubez, D. Cutts, Y.t. Duh, M. Hadley, J. Hakala, U. Heintz, J.M. $\operatorname{Hogan}^{65}$, K.H.M. Kwok, E. Laird, G. Landsberg, J. Lee, Z. Mao, M. Narain, S. Sagir ${ }^{66}$, R. Syarif, E. Usai, D. Yu

University of California, Davis, Davis, U.S.A.

R. Band, C. Brainerd, R. Breedon, M. Calderon De La Barca Sanchez, M. Chertok, J. Conway, R. Conway, P.T. Cox, R. Erbacher, C. Flores, G. Funk, F. Jensen, W. Ko, O. Kukral, R. Lander, M. Mulhearn, D. Pellett, J. Pilot, M. Shi, D. Stolp, D. Taylor,

K. Tos, M. Tripathi, Z. Wang, F. Zhang

University of California, Los Angeles, U.S.A.

M. Bachtis, C. Bravo, R. Cousins, A. Dasgupta, A. Florent, J. Hauser, M. Ignatenko, N. Mccoll, W.A. Nash, S. Regnard, D. Saltzberg, C. Schnaible, B. Stone, V. Valuev

University of California, Riverside, Riverside, U.S.A.

K. Burt, R. Clare, J.W. Gary, S.M.A. Ghiasi Shirazi, G. Hanson, G. Karapostoli, E. Kennedy, O.R. Long, M. Olmedo Negrete, M.I. Paneva, W. Si, L. Wang, H. Wei, S. Wimpenny, B.R. Yates, Y. Zhang

University of California, San Diego, La Jolla, U.S.A.

J.G. Branson, P. Chang, S. Cittolin, M. Derdzinski, R. Gerosa, D. Gilbert, B. Hashemi, D. Klein, V. Krutelyov, J. Letts, M. Masciovecchio, S. May, S. Padhi, M. Pieri, V. Sharma, M. Tadel, F. Würthwein, A. Yagil, G. Zevi Della Porta

University of California, Santa Barbara - Department of Physics, Santa Barbara, U.S.A.

N. Amin, R. Bhandari, C. Campagnari, M. Citron, V. Dutta, M. Franco Sevilla, L. Gouskos, J. Incandela, B. Marsh, H. Mei, A. Ovcharova, H. Qu, J. Richman, U. Sarica, D. Stuart, S. Wang, J. Yoo 
California Institute of Technology, Pasadena, U.S.A.

D. Anderson, A. Bornheim, O. Cerri, I. Dutta, J.M. Lawhorn, N. Lu, J. Mao, H.B. Newman, T.Q. Nguyen, J. Pata, M. Spiropulu, J.R. Vlimant, C. Wang, S. Xie, Z. Zhang, R.Y. Zhu

Carnegie Mellon University, Pittsburgh, U.S.A.

M.B. Andrews, T. Ferguson, T. Mudholkar, M. Paulini, M. Sun, I. Vorobiev, M. Weinberg

University of Colorado Boulder, Boulder, U.S.A.

J.P. Cumalat, W.T. Ford, A. Johnson, E. MacDonald, T. Mulholland, R. Patel, A. Perloff, K. Stenson, K.A. Ulmer, S.R. Wagner

Cornell University, Ithaca, U.S.A.

J. Alexander, J. Chaves, Y. Cheng, J. Chu, A. Datta, A. Frankenthal, K. Mcdermott, N. Mirman, J.R. Patterson, D. Quach, A. Rinkevicius ${ }^{67}$, A. Ryd, S.M. Tan, Z. Tao, J. Thom, P. Wittich, M. Zientek

Fermi National Accelerator Laboratory, Batavia, U.S.A.

S. Abdullin, M. Albrow, M. Alyari, G. Apollinari, A. Apresyan, A. Apyan, S. Banerjee, L.A.T. Bauerdick, A. Beretvas, J. Berryhill, P.C. Bhat, K. Burkett, J.N. Butler, A. Canepa, G.B. Cerati, H.W.K. Cheung, F. Chlebana, M. Cremonesi, J. Duarte, V.D. Elvira, J. Freeman, Z. Gecse, E. Gottschalk, L. Gray, D. Green, S. Grünendahl, O. Gutsche, AllisonReinsvold Hall, J. Hanlon, R.M. Harris, S. Hasegawa, R. Heller, J. Hirschauer, B. Jayatilaka, S. Jindariani, M. Johnson, U. Joshi, B. Klima, M.J. Kortelainen, B. Kreis, S. Lammel, J. Lewis, D. Lincoln, R. Lipton, M. Liu, T. Liu, J. Lykken, K. Maeshima, J.M. Marraffino, D. Mason, P. McBride, P. Merkel, S. Mrenna, S. Nahn, V. O’Dell, V. Papadimitriou, K. Pedro, C. Pena, G. Rakness, F. Ravera, L. Ristori, B. Schneider, E. Sexton-Kennedy, N. Smith, A. Soha, W.J. Spalding, L. Spiegel, S. Stoynev, J. Strait, N. Strobbe, L. Taylor, S. Tkaczyk, N.V. Tran, L. Uplegger, E.W. Vaandering, C. Vernieri, M. Verzocchi, R. Vidal, M. Wang, H.A. Weber

University of Florida, Gainesville, U.S.A.

D. Acosta, P. Avery, P. Bortignon, D. Bourilkov, A. Brinkerhoff, L. Cadamuro, A. Carnes, V. Cherepanov, D. Curry, F. Errico, R.D. Field, S.V. Gleyzer, B.M. Joshi, M. Kim, J. Konigsberg, A. Korytov, K.H. Lo, P. Ma, K. Matchev, N. Menendez, G. Mitselmakher, D. Rosenzweig, K. Shi, J. Wang, S. Wang, X. Zuo

Florida International University, Miami, U.S.A.

Y.R. Joshi

Florida State University, Tallahassee, U.S.A.

T. Adams, A. Askew, S. Hagopian, V. Hagopian, K.F. Johnson, R. Khurana, T. Kolberg, G. Martinez, T. Perry, H. Prosper, C. Schiber, R. Yohay, J. Zhang

Florida Institute of Technology, Melbourne, U.S.A.

M.M. Baarmand, V. Bhopatkar, M. Hohlmann, D. Noonan, M. Rahmani, M. Saunders, F. Yumiceva 
University of Illinois at Chicago (UIC), Chicago, U.S.A.

M.R. Adams, L. Apanasevich, D. Berry, R.R. Betts, R. Cavanaugh, X. Chen, S. Dittmer, O. Evdokimov, C.E. Gerber, D.A. Hangal, D.J. Hofman, K. Jung, C. Mills, T. Roy, M.B. Tonjes, N. Varelas, H. Wang, X. Wang, Z. Wu

The University of Iowa, Iowa City, U.S.A.

M. Alhusseini, B. Bilki ${ }^{51}$, W. Clarida, K. Dilsiz ${ }^{68}$, S. Durgut, R.P. Gandrajula, M. Haytmyradov, V. Khristenko, O.K. Köseyan, J.-P. Merlo, A. Mestvirishvili ${ }^{69}$, A. Moeller, J. Nachtman, H. Ogul ${ }^{70}$, Y. Onel, F. Ozok ${ }^{71}$, A. Penzo, C. Snyder, E. Tiras, J. Wetzel

Johns Hopkins University, Baltimore, U.S.A.

B. Blumenfeld, A. Cocoros, N. Eminizer, D. Fehling, L. Feng, A.V. Gritsan, W.T. Hung, P. Maksimovic, J. Roskes, M. Swartz, M. Xiao

The University of Kansas, Lawrence, U.S.A.

C. Baldenegro Barrera, P. Baringer, A. Bean, S. Boren, J. Bowen, A. Bylinkin, T. Isidori, S. Khalil, J. King, G. Krintiras, A. Kropivnitskaya, C. Lindsey, D. Majumder, W. Mcbrayer, N. Minafra, M. Murray, C. Rogan, C. Royon, S. Sanders, E. Schmitz, J.D. Tapia Takaki, Q. Wang, J. Williams, G. Wilson

Kansas State University, Manhattan, U.S.A.

S. Duric, A. Ivanov, K. Kaadze, D. Kim, Y. Maravin, D.R. Mendis, T. Mitchell, A. Modak, A. Mohammadi

Lawrence Livermore National Laboratory, Livermore, U.S.A.

F. Rebassoo, D. Wright

University of Maryland, College Park, U.S.A.

A. Baden, O. Baron, A. Belloni, S.C. Eno, Y. Feng, N.J. Hadley, S. Jabeen, G.Y. Jeng, R.G. Kellogg, J. Kunkle, A.C. Mignerey, S. Nabili, F. Ricci-Tam, M. Seidel, Y.H. Shin, A. Skuja, S.C. Tonwar, K. Wong

Massachusetts Institute of Technology, Cambridge, U.S.A.

D. Abercrombie, B. Allen, A. Baty, R. Bi, S. Brandt, W. Busza, I.A. Cali, M. D'Alfonso,

G. Gomez Ceballos, M. Goncharov, P. Harris, D. Hsu, M. Hu, M. Klute, D. Kovalskyi, Y.-J. Lee, P.D. Luckey, B. Maier, A.C. Marini, C. Mcginn, C. Mironov, S. Narayanan, X. Niu, C. Paus, D. Rankin, C. Roland, G. Roland, Z. Shi, G.S.F. Stephans, K. Sumorok, K. Tatar, D. Velicanu, J. Wang, T.W. Wang, B. Wyslouch

University of Minnesota, Minneapolis, U.S.A.

A.C. Benvenuti ${ }^{\dagger}$, R.M. Chatterjee, A. Evans, S. Guts, P. Hansen, J. Hiltbrand, S. Kalafut, Y. Kubota, Z. Lesko, J. Mans, R. Rusack, M.A. Wadud

University of Mississippi, Oxford, U.S.A.

J.G. Acosta, S. Oliveros

University of Nebraska-Lincoln, Lincoln, U.S.A.

K. Bloom, D.R. Claes, C. Fangmeier, L. Finco, F. Golf, R. Gonzalez Suarez, R. Kamalieddin, I. Kravchenko, J.E. Siado, G.R. Snow, B. Stieger 
State University of New York at Buffalo, Buffalo, U.S.A.

G. Agarwal, C. Harrington, I. Iashvili, A. Kharchilava, C. Mclean, D. Nguyen, A. Parker, J. Pekkanen, S. Rappoccio, B. Roozbahani

Northeastern University, Boston, U.S.A.

G. Alverson, E. Barberis, C. Freer, Y. Haddad, A. Hortiangtham, G. Madigan, D.M. Morse,

T. Orimoto, L. Skinnari, A. Tishelman-Charny, T. Wamorkar, B. Wang, A. Wisecarver,

D. Wood

Northwestern University, Evanston, U.S.A.

S. Bhattacharya, J. Bueghly, T. Gunter, K.A. Hahn, N. Odell, M.H. Schmitt, K. Sung, M. Trovato, M. Velasco

University of Notre Dame, Notre Dame, U.S.A.

R. Bucci, N. Dev, R. Goldouzian, M. Hildreth, K. Hurtado Anampa, C. Jessop, D.J. Karmgard, K. Lannon, W. Li, N. Loukas, N. Marinelli, I. Mcalister, F. Meng, C. Mueller, Y. Musienko ${ }^{35}$, M. Planer, R. Ruchti, P. Siddireddy, G. Smith, S. Taroni, M. Wayne, A. Wightman, M. Wolf, A. Woodard

The Ohio State University, Columbus, U.S.A.

J. Alimena, B. Bylsma, L.S. Durkin, S. Flowers, B. Francis, C. Hill, W. Ji, A. Lefeld, T.Y. Ling, B.L. Winer

Princeton University, Princeton, U.S.A.

S. Cooperstein, G. Dezoort, P. Elmer, J. Hardenbrook, N. Haubrich, S. Higginbotham, A. Kalogeropoulos, S. Kwan, D. Lange, M.T. Lucchini, J. Luo, D. Marlow, K. Mei, I. Ojalvo, J. Olsen, C. Palmer, P. Piroué, J. Salfeld-Nebgen, D. Stickland, C. Tully, Z. Wang

University of Puerto Rico, Mayaguez, U.S.A.

S. Malik, S. Norberg

Purdue University, West Lafayette, U.S.A.

A. Barker, V.E. Barnes, S. Das, L. Gutay, M. Jones, A.W. Jung, A. Khatiwada, B. Mahakud, D.H. Miller, G. Negro, N. Neumeister, C.C. Peng, S. Piperov, H. Qiu, J.F. Schulte, J. Sun, F. Wang, R. Xiao, W. Xie

Purdue University Northwest, Hammond, U.S.A.

T. Cheng, J. Dolen, N. Parashar

Rice University, Houston, U.S.A.

K.M. Ecklund, S. Freed, F.J.M. Geurts, M. Kilpatrick, Arun Kumar, W. Li, B.P. Padley, R. Redjimi, J. Roberts, J. Rorie, W. Shi, A.G. Stahl Leiton, Z. Tu, A. Zhang

University of Rochester, Rochester, U.S.A.

A. Bodek, P. de Barbaro, R. Demina, J.L. Dulemba, C. Fallon, T. Ferbel, M. Galanti, A. Garcia-Bellido, J. Han, O. Hindrichs, A. Khukhunaishvili, E. Ranken, P. Tan, R. Taus 
Rutgers, The State University of New Jersey, Piscataway, U.S.A.

B. Chiarito, J.P. Chou, A. Gandrakota, Y. Gershtein, E. Halkiadakis, A. Hart, M. Heindl, E. Hughes, S. Kaplan, S. Kyriacou, I. Laflotte, A. Lath, R. Montalvo, K. Nash, M. Osherson, H. Saka, S. Salur, S. Schnetzer, D. Sheffield, S. Somalwar, R. Stone, S. Thomas, P. Thomassen

University of Tennessee, Knoxville, U.S.A.

H. Acharya, A.G. Delannoy, J. Heideman, G. Riley, S. Spanier

Texas A\&M University, College Station, U.S.A.

O. Bouhali ${ }^{72}$, A. Celik, M. Dalchenko, M. De Mattia, A. Delgado, S. Dildick, R. Eusebi, J. Gilmore, T. Huang, T. Kamon ${ }^{73}$, S. Luo, D. Marley, R. Mueller, D. Overton, L. Perniè, D. Rathjens, A. Safonov

\section{Texas Tech University, Lubbock, U.S.A.}

N. Akchurin, J. Damgov, F. De Guio, S. Kunori, K. Lamichhane, S.W. Lee, T. Mengke, S. Muthumuni, T. Peltola, S. Undleeb, I. Volobouev, Z. Wang, A. Whitbeck

Vanderbilt University, Nashville, U.S.A.

S. Greene, A. Gurrola, R. Janjam, W. Johns, C. Maguire, A. Melo, H. Ni, K. Padeken, F. Romeo, P. Sheldon, S. Tuo, J. Velkovska, M. Verweij

University of Virginia, Charlottesville, U.S.A.

M.W. Arenton, P. Barria, B. Cox, G. Cummings, R. Hirosky, M. Joyce, A. Ledovskoy, C. Neu, B. Tannenwald, Y. Wang, E. Wolfe, F. Xia

\section{Wayne State University, Detroit, U.S.A.}

R. Harr, P.E. Karchin, N. Poudyal, J. Sturdy, P. Thapa, S. Zaleski

University of Wisconsin - Madison, Madison, WI, U.S.A.

J. Buchanan, C. Caillol, D. Carlsmith, S. Dasu, I. De Bruyn, L. Dodd, F. Fiori, C. Galloni, B. Gomber ${ }^{74}$, M. Herndon, A. Hervé, U. Hussain, P. Klabbers, A. Lanaro, A. Loeliger, K. Long, R. Loveless, J. Madhusudanan Sreekala, T. Ruggles, A. Savin, V. Sharma, W.H. Smith, D. Teague, S. Trembath-reichert, N. Woods

$\dagger$ : Deceased

1: Also at Vienna University of Technology, Vienna, Austria

2: Also at IRFU, CEA, Université Paris-Saclay, Gif-sur-Yvette, France

3: Also at Universidade Estadual de Campinas, Campinas, Brazil

4: Also at Federal University of Rio Grande do Sul, Porto Alegre, Brazil

5: Also at UFMS/CPNA - Federal University of Mato Grosso do Sul/Campus of Nova Andradina, Nova Andradina, Brazil

6: Also at Universidade Federal de Pelotas, Pelotas, Brazil

7: Also at Université Libre de Bruxelles, Bruxelles, Belgium

8: Also at University of Chinese Academy of Sciences, Beijing, China

9: Also at Institute for Theoretical and Experimental Physics named by A.I. Alikhanov of NRC 'Kurchatov Institute', Moscow, Russia

10: Also at Joint Institute for Nuclear Research, Dubna, Russia 
11: Also at Suez University, Suez, Egypt

12: Now at British University in Egypt, Cairo, Egypt

13: Also at Purdue University, West Lafayette, U.S.A.

14: Also at Université de Haute Alsace, Mulhouse, France

15: Also at Erzincan Binali Yildirim University, Erzincan, Turkey

16: Also at CERN, European Organization for Nuclear Research, Geneva, Switzerland

17: Also at RWTH Aachen University, III. Physikalisches Institut A, Aachen, Germany

18: Also at University of Hamburg, Hamburg, Germany

19: Also at Brandenburg University of Technology, Cottbus, Germany

20: Also at Institute of Physics, University of Debrecen, Debrecen, Hungary

21: Also at Institute of Nuclear Research ATOMKI, Debrecen, Hungary

22: Also at MTA-ELTE Lendület CMS Particle and Nuclear Physics Group, Eötvös Loránd University, Budapest, Hungary

23: Also at Indian Institute of Technology Bhubaneswar, Bhubaneswar, India

24: Also at Institute of Physics, Bhubaneswar, India

25: Also at Shoolini University, Solan, India

26: Also at University of Visva-Bharati, Santiniketan, India

27: Also at Isfahan University of Technology, Isfahan, Iran

28: Also at Italian National Agency for New Technologies, Energy and Sustainable Economic Development, Bologna, Italy

29: Also at Centro Siciliano di Fisica Nucleare e di Struttura della Materia, Catania, Italy

30: Also at Scuola Normale e Sezione dell'INFN, Pisa, Italy

31: Also at Riga Technical University, Riga, Latvia

32: Also at Malaysian Nuclear Agency, MOSTI, Kajang, Malaysia

33: Also at Consejo Nacional de Ciencia y Tecnología, Mexico City, Mexico

34: Also at Warsaw University of Technology, Institute of Electronic Systems, Warsaw, Poland

35: Also at Institute for Nuclear Research, Moscow, Russia

36: Now at National Research Nuclear University 'Moscow Engineering Physics Institute' (MEPhI), Moscow, Russia

37: Also at St. Petersburg State Polytechnical University, St. Petersburg, Russia

38: Also at University of Florida, Gainesville, U.S.A.

39: Also at Imperial College, London, United Kingdom

40: Also at P.N. Lebedev Physical Institute, Moscow, Russia

41: Also at California Institute of Technology, Pasadena, U.S.A.

42: Also at Budker Institute of Nuclear Physics, Novosibirsk, Russia

43: Also at Faculty of Physics, University of Belgrade, Belgrade, Serbia

44: Also at Università degli Studi di Siena, Siena, Italy

45: Also at INFN Sezione di Pavia ${ }^{a}$, Università di Pavia ${ }^{b}$, Pavia, Italy

46: Also at National and Kapodistrian University of Athens, Athens, Greece

47: Also at Universität Zürich, Zurich, Switzerland

48: Also at Stefan Meyer Institute for Subatomic Physics (SMI), Vienna, Austria

49: Also at Adiyaman University, Adiyaman, Turkey

50: Also at Sirnak University, SIRNAK, Turkey

51: Also at Beykent University, Istanbul, Turkey

52: Also at Istanbul Aydin University, Istanbul, Turkey

53: Also at Mersin University, Mersin, Turkey

54: Also at Piri Reis University, Istanbul, Turkey

55: Also at Gaziosmanpasa University, Tokat, Turkey 
56: Also at Ozyegin University, Istanbul, Turkey

57: Also at Izmir Institute of Technology, Izmir, Turkey

58: Also at Kafkas University, Kars, Turkey

59: Also at Istanbul University, Istanbul, Turkey

60: Also at Istanbul Bilgi University, Istanbul, Turkey

61: Also at Hacettepe University, Ankara, Turkey

62: Also at School of Physics and Astronomy, University of Southampton, Southampton, United Kingdom

63: Also at Institute for Particle Physics Phenomenology Durham University, Durham, United Kingdom

64: Also at Monash University, Faculty of Science, Clayton, Australia

65: Also at Bethel University, St. Paul, U.S.A.

66: Also at Karamanoğlu Mehmetbey University, Karaman, Turkey

67: Also at Vilnius University, Vilnius, Lithuania

68: Also at Bingol University, Bingol, Turkey

69: Also at Georgian Technical University, Tbilisi, Georgia

70: Also at Sinop University, Sinop, Turkey

71: Also at Mimar Sinan University, Istanbul, Istanbul, Turkey

72: Also at Texas A\&M University at Qatar, Doha, Qatar

73: Also at Kyungpook National University, Daegu, Korea

74: Also at University of Hyderabad, Hyderabad, India 